This is a peer-reviewed, un-copyedited version of an article published in Nonlinearity. IOP Publishing Ltd is not responsible for any errors or omissions in this version of the manuscript or any version derived from it. The Version of Record is available online at https://doi.org/10.1088/1361-6544/ab1bc6.

\title{
PERIODIC SOLUTIONS, KAM TORI AND BIFURCATIONS IN A COSMOLOGY-INSPIRED POTENTIAL
}

\author{
J.F. PALACIÁN, C. VIDAL, J. VIDARTE, AND P. YANGUAS
}

\begin{abstract}
A family of perturbed Hamiltonians $\mathcal{H}_{\varepsilon}=\frac{1}{2}\left(x^{2}+X^{2}\right)-\frac{1}{2}\left(y^{2}+Y^{2}\right)+\frac{1}{2}\left(z^{2}+Z^{2}\right)+$ $\varepsilon^{2}\left[\alpha\left(x^{4}+y^{4}+z^{4}\right)+\beta\left(x^{2} y^{2}+x^{2} z^{2}+y^{2} z^{2}\right)\right]$ in 1:-1:1 resonance depending on two real parameters is considered. We show the existence and stability of periodic solutions using reduction and averaging. In fact, there are at most thirteen families for every energy level $h<0$ and at most twenty six families for every $h>0$. The different types of periodic solutions for every nonzero energy level, as well as their bifurcations, are characterised in terms of the parameters. The linear stability of each family of periodic solutions, together with the determination of KAM 3-tori encasing some of the linearly stable periodic solutions is proved. Critical Hamiltonian bifurcations on the reduced space are characterised. We find important differences with respect to the dynamics of the 1:1:1 resonance with the same perturbation as the one given here. We end up with an intuitive interpretation of the results from a cosmological viewpoint.
\end{abstract}

\section{INTRODUCTION}

We consider the Hamiltonian

$$
\begin{aligned}
\mathcal{H}_{\varepsilon}= & \frac{1}{2}\left(x^{2}+X^{2}\right)-\frac{1}{2}\left(y^{2}+Y^{2}\right)+\frac{1}{2}\left(z^{2}+Z^{2}\right)+ \\
& \varepsilon^{2}\left[\alpha\left(x^{4}+y^{4}+z^{4}\right)+\beta\left(x^{2} y^{2}+x^{2} z^{2}+y^{2} z^{2}\right)\right],
\end{aligned}
$$

which consists of a $3 D$ harmonic oscillator in 1:-1:1 resonance plus a homogeneous triaxial potential of degree four depending on two real parameters $\alpha$ and $\beta$. The parameter $\varepsilon$ is supposed to be a positive small constant. This Hamiltonian is invariant with respect to the transformation $R_{\pi}$ : $(x, y, z, X, Y, Z) \rightarrow(z, y, x, Z, Y, X)$. We denote the quadratic part of Hamiltonian (1) by $\mathcal{H}_{0}$.

The unperturbed part of Hamiltonian (1) is equivalent to

$$
X y-x Y+\frac{1}{2}\left(z^{2}+Z^{2}\right)
$$

after applying a linear symplectic change of coordinates, as it can be seen in [32]. In that paper, several models that can be expressed as perturbations of the Coriolis-like term $X y-x Y$ are considered. In this sense, our present study could be also useful to model similar problems by taking into account the influence of the third degree of freedom.

When $z=Z=0$ Hamiltonian (1) is associated to a model of universe defined by the FriedmannLemaître-Robertson-Walker (FLRW) metric [15, 16, 23, 24, 41, 42, 43, 48]. It describes a homogeneous and isotropic expanding/contracting universe. The metric is given as a second-order ordinary differential equation where the independent variable is time and there are two dependent variables, basically, the scale factor and the density of the ideal fluid that fills the universe. The scale factor

1991 Mathematics Subject Classification. 34C15, 34C20, 34C25, 37J40, 70K65.

Key words and phrases. Resonant Hamiltonians in 1:-1:1 resonance; cosmological Hamiltonian; FriedmannLemaître-Robertson-Walker model; normalisation and reduction; averaging; reduced space and invariants; Reeb's Theorem; periodic solutions and linear stability; Hamiltonian Hopf bifurcation; KAM tori.

The authors are partially supported by Projects MTM 2011-28227-C02-01 of the Ministry of Science and Innovation of Spain, MTM 2014-59433-C2-1-P of the Ministry of Economy and Competitiveness of Spain and MTM 2017-88137C2-1-P of the Ministry of Science, Innovation and Universities of Spain. C. Vidal is partially supported by Project Fondecyt 1180288. 
depends on time, thence the distance between any given two points on the space also changes with the same rate. This is the essence of the FLRW universe model. The scale factor relates the physical distance to the co-moving distance and gives us the size of the observable universe. The dynamics of the FLRW universe determines the time evolution for the scale factor and for the energy density. The reader can consult [27] for a beautiful introduction, description, physical setting of the FLRW model and derivation of the Hamiltonian. That paper deals with the integrability of the system.

The metric depends on some constants, the universe curvature, among others. This model represents a first approximation to the Big Bang cosmological model. In spite of its simplicity, it reproduces basic features of the observable universe. The elementary idea underlying the model is that the universe oscillates from the Big Bang, where the density is infinite and starts to decrease, whereas the scale factor is initially zero and starts to increase.

Chaos has been found in the dynamics of the FLRW universe, see for instance [3]. The authors prove both analytically and numerically the existence of chaotic motion in the associated Hamiltonian. They transform the original system in such a way that it can be expressed as a perturbation of two oscillators in 1:-1 resonance. Planar generalisations of this model have been tackled by several authors, for example [26] uses classical averaging theory to prove the existence of three families of periodic solutions in each nonzero energy level. In [12] the authors introduce the planar FLRW Hamiltonian in rotating coordinates and using averaging theory show the existence of two families of periodic solutions for angular velocity $\omega=0$ and six families of periodic solutions for $\omega \neq 0$ in each nonzero energy level. In [45] the authors consider the formal stability of the origin of a Hamiltonian system composed by an oscillator in 1:-1:1 resonance plus a quartic perturbation.

Other authors $[20,21,22,37]$ consider also the FLRW model in the area of quantum cosmology for understanding the relation between the thermodynamic and cosmological arrows of time.

The dynamics of perturbed Hamiltonians in 1:-1:1 resonance has not been very studied. The Hamiltonian tackled in this work corresponds to this type of resonance and is a particular case of the one dealt with in [25]. There the authors, using averaging theory show the existence of four families of periodic solutions in every energy level $h \neq 0$. The linear stability of these periodic solutions is not analysed.

In the present work, using orbit-space reduction [46, 6], that is dual to Meyer-Marsden-Weinstein reduction [30, 29], and averaging in the Hamiltonian setting [36] (see also [5, 44, 50, 32, 6]), we find at most thirteen families of periodic solutions for every energy level $h<0$ and at most twenty six families of periodic solutions for every $h>0$. Moreover we study the bifurcation curves of the periodic solutions as functions of the parameters taking into account the sign of every energy level. We provide as well the linear stability or instability of the solutions, applying the notion of parametric (or strong) stability [49]. In addition to this, we study the existence of KAM threedimensional tori enclosing some of these solutions for every energy level $h>0$. For $h<0$ there is only one family of 3 -tori for a specific value of the parameters.

Our results allow us to deal with all kind of motions provided $\varepsilon$ is small enough. This is possible because we use a set of global coordinates that parametrise the reduced space properly. These coordinates are the invariants associated to the oscillator symmetry, which is the symmetry the normalised system possesses after truncating higher-order terms. Specifically, for the 1:-1:1 resonance the number of invariants is nine, the minimum possible for a resonant Hamiltonian with three degrees of freedom.

In this setting, we can discuss the number of zeroes of the reduced vector field which is a polynomial system in terms of the nine invariants plus three constants, namely, the parameters $\alpha$ and $\beta$ and the constant $h$. To this system we need to attach the existing constraints relating the invariants. Once the appearance or disappearance of the critical points is analysed one needs to define symplectic variables (four coordinates) to perform a local study around each critical point. This allows one to go through the stability character of the equilibrium, leading to deciding on the 
linear stability of the corresponding periodic solutions and determining, in cases of non-degeneracy, invariant 3-tori enclosing the linearly stable periodic solutions for every energy level $h>0$.

This paper is organised as follows. In Section 2 we calculate the normal form of $\mathcal{H}_{\varepsilon}$ up to order four in rectangular coordinates and we introduce the invariants associated with the quadratic part. Thence, we write the normalised Hamiltonian in terms of the invariants and after fixing an energy level, $\mathcal{H}_{0}=h \neq 0$, we determine the reduced space, the reduced Hamiltonian, as well as the associated Poisson vector field. This vector field has at most thirteen critical points defined for an energy level $h<0$ and at most twenty six critical points for an energy level $h>0$. Then, taking into account that the foliation of the orbit space into symplectic leaves is regular for $h \neq 0$ $[30,29,50]$ these points are in correspondence with periodic solutions of the truncated Hamiltonian system in normal form up to the order of the approximation. In particular, a critical point in the vector field written in terms of the invariants determines a family of periodic solutions parametrised by the energy $h \neq 0$. Each of these solutions is a candidate to be continued and to give rise to a family of periodic solutions of the full system.

In Section 3 we construct three different families of symplectic coordinates on the reduced space taking into consideration the sign of every energy level. This is achieved by defining symplectic coordinates $\left(L, Q_{1}, Q_{2}, \ell, P_{1}, P_{2}\right)$ in $\mathbb{R}^{6}$ that reflect the normalisation process, i.e., such that the quadratic part of (1) in the new coordinates simply depends on a single action $L$ and the truncated normalised Hamiltonian (or averaged system with respect to the angle $\ell$ ) has $L$ as a first integral. Then, fixing $\mathcal{H}_{0}=L=h$ we obtain a Hamiltonian with two degrees of freedom on the reduced space. This Hamiltonian is the same as the one obtained in terms of the invariants. The remaining variables $\left(Q_{1}, Q_{2}, P_{1}, P_{2}\right)$ can be put in terms of the invariants; therefore, they are good coordinates to study the critical points previously obtained.

The existence of families of periodic solutions in terms of $\alpha$ and $\beta$ as well as their linear stability are treated in Section 4. Now, the critical points obtained as functions of the invariants are written in terms of the coordinates $\left(Q_{1}, Q_{2}, P_{1}, P_{2}\right)$ and they naturally correspond to critical points of the averaged Hamiltonian. By virtue of Theorem A.2 (see [50]), that is a modern formulation of Reeb's Theorem [39, 40], we obtain conditions on the parameters $\alpha$ and $\beta$ for the critical points to be nondegenerate, giving rise to families of periodic solutions of the full system. Next, using the notion of strong stability and Krein-Gel'fand theory [49] we conclude the linear stability or instability of the periodic solutions.

Some Hamiltonian bifurcations for negative and positive energy levels are characterised in Section 5. The main finding in this respect is a periodic Hamiltonian Hopf bifurcations of the system associated to (1) related to the rectilinear periodic solutions in the $O y$ axis for $h<0$. When $h>0$ we analyse with detail the bifurcations of the solutions of rectilinear type in the $O x$ and $O z$ axes as well as the bifurcation corresponding to the circular motions on the plane $O x z$. We content ourselves to perform the study in the reduced space without reconstructing the dynamics of the full system since, to our knowledge, there are no theorems in the literature that can be applied in our setting.

Section 6 is devoted to establishing the persistence of KAM 3-tori encasing some elliptic periodic solutions applying KAM theory [1]. More precisely, we make use of Han, Li and Yi's Theorem A.4 (see details in [17]) that applies in the case of Hamiltonian systems with high-order proper degeneracy.

Finally, some conclusions are presented and, in order to make the paper self-contained, we include Appendix A, where we have collected the results on averaging theory, KAM theory and strong stability for Hamiltonian systems that we use throughout the paper. Appendix B contains the tables accounting for the relative equilibria written in the invariants, as well as in different charts. 
Our main achievements are presented in Sections 4, 5 and 6 by means of Theorems 4.1, 4.2, 4.3, 4.4, 5.1 and 6.1 where we establish the results on the periodic solutions, the Hamiltonian Hopf bifurcations and the KAM 3-tori.

\section{Normalisation AND REDUCTION}

In order to describe the orbit space of the isotropic oscillator $\mathcal{H}_{0}$, we identify $\mathbb{R}^{6}$ with $\mathbb{C}^{3}$ through the change of variables $\zeta_{1}=x+X i, \zeta_{2}=y+Y i$ and $\zeta_{3}=z+Z i$. Thus, the unperturbed Hamiltonian $\mathcal{H}_{0}$ written in terms of $\zeta=\left(\zeta_{1}, \zeta_{2}, \zeta_{3}\right)$ takes the form

$$
\mathcal{H}_{0}(\zeta)=\frac{1}{2}\left(\left|\zeta_{1}\right|^{2}-\left|\zeta_{2}\right|^{2}+\left|\zeta_{3}\right|^{2}\right) \text {. }
$$

The flow of $\mathcal{H}_{0}$ induces the action $\varphi: S^{1} \times \mathbb{C}^{3} \rightarrow \mathbb{C}^{3}$ defined by $\varphi_{t}(\zeta)=\left(e^{t i} \zeta_{1}, e^{-t i} \zeta_{2}, e^{t i} \zeta_{3}\right)$, which for $t \in[0,2 \pi)$ is a free and proper $S^{1}$-action on any level surface $\mathcal{N}_{0}(h)=\mathcal{H}_{0}^{-1}(h) \subset \mathbb{R}^{6}$ for $h \neq 0$. Since $\mathcal{N}_{0}(h)$ is the 5 -manifold

$$
\mathcal{M}_{h}^{5}=\left\{\zeta \in \mathbb{C}^{3}: \mathcal{H}_{0}(\zeta)=2 h\right\}
$$

then the orbit space of the $S^{1}$ action on $\mathcal{N}_{0}(h)$ (also called the reduced space) is $\mathcal{M}_{h}^{5} / S^{1}$. This space, that we denote by $\mathcal{B}(h)$, is a symplectic non-compact manifold of dimension four. This is the first difference with respect to the 1:1:1 resonance, where the base space is compact; however we are also in the case of regular reduction [30, 29].

Next, with the goal of using the symmetry of the oscillator, we normalise Hamiltonian (1) up to order four in the small parameter applying the Lie-Deprit method [7]. In rectangular coordinates the normalised Hamiltonian (1) is

$$
\begin{aligned}
\mathcal{H}_{\varepsilon}=\mathcal{H}_{0}+\varepsilon^{2}\{ & \frac{3}{8} \alpha\left(x^{2}-y^{2}+z^{2}+X^{2}-Y^{2}+Z^{2}\right)^{2}+ \\
& \frac{3}{8}(2 \alpha+\beta)\left[(x y-X Y)^{2}+(y z-Y Z)^{2}\right]+\frac{3}{8}(\beta-2 \alpha)(x z+X Z)^{2}+ \\
& \left.\frac{1}{8}(6 \alpha+\beta)\left[(x Y+y X)^{2}+(y Z+z Y)^{2}\right]+\frac{1}{8}(\beta-6 \alpha)(x Z-z X)^{2}\right\}+O\left(\varepsilon^{4}\right) .
\end{aligned}
$$

The orbit space $\mathcal{B}(h)$ is properly described by the quadratic constants of motion associated to the oscillator, that are called the invariants of the 1:-1:1 resonance. In particular the invariants are quadratic polynomials (that we denote by $\left.\pi_{1}, \pi_{2}, \ldots, \pi_{9}\right)$ in the coordinates $(x, y, z, X, Y, Z)$ that generate the space of functions with respect to the action given by the flow of $\mathcal{H}_{0}$, thence $\left\{\mathcal{H}_{0}, \pi_{j}\right\}=0$, for all $j=1, \ldots, 9$, where $\{$,$\} is the standard Poisson bracket of two functions.$ Explicitly, the invariants are

$$
\begin{array}{lll}
\pi_{1}=x^{2}+X^{2}, & \pi_{2}=y^{2}+Y^{2}, & \pi_{3}=z^{2}+Z^{2}, \\
\pi_{4}=x y-X Y, & \pi_{5}=x z+X Z, & \pi_{6}=y z-Y Z, \\
\pi_{7}=x Y+y X, & \pi_{8}=x Z-z X, & \pi_{9}=y Z+z Y .
\end{array}
$$

Note that these invariants are different from the ones of the 1:1:1 resonance (see [38]). The normalised Hamiltonian (3) can be expressed in terms of the invariants. This is usually achieved using strategies from computer algebra, see the details in [33, 34], although in the particular case of (3), it can be done straightforwardly.

Some relations of dependence of degree one or two among the invariants are:

$$
\begin{array}{lll} 
& \pi_{1}-\pi_{2}+\pi_{3}=2 h, & \\
\pi_{1} \pi_{2}=\pi_{4}^{2}+\pi_{7}^{2}, & \pi_{1} \pi_{3}=\pi_{5}^{2}+\pi_{8}^{2}, & \pi_{2} \pi_{3}=\pi_{6}^{2}+\pi_{9}^{2}, \\
\pi_{1} \pi_{6}=\pi_{4} \pi_{5}-\pi_{7} \pi_{8}, & \pi_{2} \pi_{8}=\pi_{4} \pi_{9}-\pi_{6} \pi_{7}, & \pi_{3} \pi_{4}=\pi_{5} \pi_{6}+\pi_{8} \pi_{9}, \\
\pi_{4} \pi_{6}=\pi_{2} \pi_{5}-\pi_{7} \pi_{9}, & \pi_{4} \pi_{8}=\pi_{1} \pi_{9}-\pi_{5} \pi_{7}, & \pi_{6} \pi_{8}=-\pi_{3} \pi_{7}+\pi_{5} \pi_{9} .
\end{array}
$$

Besides, the following inequalities have to be satisfied:

$$
\pi_{1} \geq 0, \quad \pi_{2} \geq 0, \quad \pi_{3} \geq 0
$$


Indeed, as $\mathcal{B}(h)$ is four-dimensional, in (5) there must be five functionally independent constraints among the $\pi_{i}$, see the details in [14]. Table 1 contains the Poisson structure of the invariants. These Poisson brackets describe the Lie algebra formed by the invariants.

\begin{tabular}{|c|c|c|c|c|c|c|c|c|c|}
\hline$\left\{\pi_{j}, \pi_{k}\right\}$ & $\pi_{1}$ & $\pi_{2}$ & $\pi_{3}$ & $\pi_{4}$ & $\pi_{5}$ & $\pi_{6}$ & $\pi_{7}$ & $\pi_{8}$ & $\pi_{9}$ \\
\hline$\pi_{1}$ & 0 & 0 & 0 & $-2 \pi_{7}$ & $2 \pi_{8}$ & 0 & $2 \pi_{4}$ & $-2 \pi_{5}$ & 0 \\
\hline$\pi_{2}$ & 0 & 0 & 0 & $-2 \pi_{7}$ & 0 & $-2 \pi_{9}$ & $2 \pi_{4}$ & 0 & $2 \pi_{6}$ \\
\hline$\pi_{3}$ & 0 & 0 & 0 & 0 & $-2 \pi_{8}$ & $-2 \pi_{9}$ & 0 & $2 \pi_{5}$ & $2 \pi_{6}$ \\
\hline$\pi_{4}$ & $2 \pi_{7}$ & $2 \pi_{7}$ & 0 & 0 & $\pi_{9}$ & $-\pi_{8}$ & $\pi_{1}+\pi_{2}$ & $-\pi_{6}$ & $\pi_{5}$ \\
\hline$\pi_{5}$ & $-2 \pi_{8}$ & 0 & $2 \pi_{8}$ & $-\pi_{9}$ & 0 & $-\pi_{7}$ & $\pi_{6}$ & $\pi_{1}-\pi_{3}$ & $\pi_{4}$ \\
\hline$\pi_{6}$ & 0 & $2 \pi_{9}$ & $2 \pi_{9}$ & $\pi_{8}$ & $\pi_{7}$ & 0 & $\pi_{5}$ & $\pi_{4}$ & $\pi_{2}+\pi_{3}$ \\
\hline$\pi_{7}$ & $-2 \pi_{4}$ & $-2 \pi_{4}$ & 0 & $-\pi_{1}-\pi_{2}$ & $-\pi_{6}$ & $-\pi_{5}$ & 0 & $-\pi_{9}$ & $-\pi_{8}$ \\
\hline$\pi_{8}$ & $2 \pi_{5}$ & 0 & $-2 \pi_{5}$ & $\pi_{6}$ & $\pi_{3}-\pi_{1}$ & $-\pi_{4}$ & $\pi_{9}$ & 0 & $-\pi_{7}$ \\
\hline$\pi_{9}$ & 0 & $-2 \pi_{6}$ & $-2 \pi_{6}$ & $-\pi_{5}$ & $-\pi_{4}$ & $-\pi_{2}-\pi_{3}$ & $\pi_{8}$ & $\pi_{7}$ & 0 \\
\hline
\end{tabular}

TABLE 1. Poisson brackets among the invariants $\pi_{i}$.

Hamiltonian (3) in terms of the invariant coordinates becomes

$$
\mathcal{H}_{\varepsilon}(\pi)=\mathcal{H}_{0}(\pi)+\varepsilon^{2} \mathcal{H}_{2}(\pi)+O\left(\varepsilon^{4}\right)
$$

where

$$
\begin{aligned}
\mathcal{H}_{0}(\pi)= & \frac{1}{2}\left(\pi_{1}-\pi_{2}+\pi_{3}\right), \\
\mathcal{H}_{2}(\pi)= & \frac{3}{8} \alpha\left(\pi_{1}-\pi_{2}+\pi_{3}\right)^{2}+\frac{3}{8}(2 \alpha+\beta)\left(\pi_{4}^{2}+\pi_{6}^{2}\right)+\frac{3}{8}(\beta-2 \alpha) \pi_{5}^{2}+ \\
& \frac{1}{8}(6 \alpha+\beta)\left(\pi_{7}^{2}+\pi_{9}^{2}\right)+\frac{1}{8}(\beta-6 \alpha) \pi_{8}^{2} .
\end{aligned}
$$

Next, we take into account that the vector field associated to $(7)$ in the variables $\left(\pi_{1}, \pi_{2}, \ldots, \pi_{9}\right)$ is given by

$$
\frac{d \pi_{j}}{d t}=\left\{\pi_{j}, \mathcal{H}_{\varepsilon}\right\}=\sum_{k=1}^{9}\left\{\pi_{j}, \pi_{k}\right\} \frac{\partial \mathcal{H}_{\varepsilon}}{\partial \pi_{k}}, \quad j=1, \ldots, 9
$$

The reduction is now performed by introducing the Hilbert map $\rho_{\pi}: \mathbb{R}^{6} \rightarrow \mathbb{R}^{9}$ defined by $\rho_{\pi}(x, y, z, X, Y, Z)=\left(\pi_{1}, \ldots, \pi_{9}\right)$. The image of this map is the orbit space for the $\mathcal{H}_{0}$-action and the image of a level surface $\mathcal{N}_{0}(h)$ under $\rho_{\pi}$ is the reduced space, i.e., $\mathcal{B}(h)=\rho_{\pi}\left(\mathcal{N}_{0}(h)\right)$. In this way, fixing a constant value $h \neq 0$, the reduced space $\mathcal{B}(h)$ is given by the constraints $(5)$ - we recall that only five of them are functionally independent - and (6).

The reduced Hamiltonian is obtained from (7) after truncating terms of order $\varepsilon^{4}$ and higher, dropping the constant terms and dividing the resulting Hamiltonian by $\varepsilon^{2}$, that is,

$$
\overline{\mathcal{H}}=\frac{3}{8}(2 \alpha+\beta)\left(\pi_{4}^{2}+\pi_{6}^{2}\right)+\frac{3}{8}(\beta-2 \alpha) \pi_{5}^{2}+\frac{1}{8}(6 \alpha+\beta)\left(\pi_{7}^{2}+\pi_{9}^{2}\right)+\frac{1}{8}(\beta-6 \alpha) \pi_{8}^{2}
$$


The equations of motion for Hamiltonian (9) are

$$
\begin{aligned}
& \dot{\pi_{1}}=-\beta\left(\pi_{4} \pi_{7}-\pi_{5} \pi_{8}\right), \\
& \dot{\pi_{2}}=-\beta\left(\pi_{4} \pi_{7}+\pi_{6} \pi_{9}\right), \\
& \dot{\pi_{3}}=-\beta\left(\pi_{5} \pi_{8}+\pi_{6} \pi_{9}\right), \\
& \dot{\pi_{4}}=\frac{1}{4}(6 \alpha+\beta)\left(2 h+2 \pi_{2}-\pi_{3}\right) \pi_{7}+\beta\left(\pi_{5} \pi_{9}-\pi_{6} \pi_{8}\right), \\
& \dot{\pi_{5}}=\frac{1}{4}(\beta-6 \alpha)\left(2 h+\pi_{2}-2 \pi_{3}\right) \pi_{8}-\frac{1}{2} \beta\left(\pi_{4} \pi_{9}+\pi_{6} \pi_{7}\right), \\
& \dot{\pi_{6}}=\frac{1}{4}(6 \alpha+\beta)\left(\pi_{2}+\pi_{3}\right) \pi_{9}+\beta\left(\pi_{4} \pi_{8}+\pi_{5} \pi_{7}\right), \\
& \dot{\pi_{7}}=-\frac{3}{4}(2 \alpha+\beta)\left(2 h+2 \pi_{2}-\pi_{3}\right) \pi_{4}-\frac{1}{2} \beta\left(3 \pi_{5} \pi_{6}+\pi_{8} \pi_{9}\right), \\
& \dot{\pi_{8}}=-\frac{3}{4}(\beta-2 \alpha)\left(2 h+\pi_{2}-2 \pi_{3}\right) \pi_{5}, \\
& \dot{\pi_{9}}=-\frac{3}{4}(2 \alpha+\beta)\left(\pi_{2}+\pi_{3}\right) \pi_{6}-\frac{1}{2} \beta\left(3 \pi_{4} \pi_{5}-\pi_{7} \pi_{8}\right) .
\end{aligned}
$$

Proposition 2.1. On the reduced space $\mathcal{B}(h)$ with $h<0$, the system (10) has at most thirteen isolated critical points (see Table 6 in Appendix B). More precisely, if we set $\delta=6 \alpha / \beta$ :

(i) If $\delta \in D_{1}=(-\infty,-5] \cup[-3,-7 / 3] \cup[-1, \infty)$ there is only one critical point, the one corresponding to motions of rectilinear type in the $O y$ axis.

(ii) If $\delta \in D_{2}=(-5,-(3+4 \sqrt{3}) / 3]$ or $\delta \in D_{3}=(-7 / 3,-1)$ there are five critical points.

(iii) If $\delta \in D_{4}=(-(3+4 \sqrt{3}) / 3,-3)$ there are thirteen critical points.

(iv) If $\beta=0$ and $\alpha \neq 0$ there is only one critical point that corresponds to point 1) of Table 6 in Appendix B.

Proof. System (10) has to be solved taking into account the restrictions of (5) and (6). Using Mathematica we get the isolated equilibria appearing in Table 6 in Appendix B.

Proposition 2.2. On the reduced space $\mathcal{B}(h)$ with $h>0$, the system (10) has at most twenty six isolated critical points (see Table 7 in Appendix B). More precisely, if we set $\delta=6 \alpha / \beta$ :

(i) If $\delta \in D_{5}=(-\infty,-9] \cup\{-5\} \cup[-7 / 3,+\infty)$ there are six critical points.

(ii) If $\delta \in D_{6}=(-9,-5)$ there are ten critical points.

(iii) If $\delta \in D_{7}=[-(3+4 \sqrt{3}) / 3,-7 / 3)$ there are eighteen critical points.

(iv) If $\delta \in D_{8}=(-5,-(3+4 \sqrt{3}) / 3)$ there are twenty six critical points.

(v) If $\beta=0$ and $\alpha \neq 0$ there are two critical points that correspond to points 1) and 2) of Table 7 in Appendix $B$.

Proof. System (10) is solved by using Mathematica. Taking into account the restrictions appearing in (5) and (6) we end up with the occurrence of the critical points for the different values of $\delta$ listed above.

Observation 2.1. The critical points of Propositions 2.1 and 2.2 are the candidates to get periodic solutions of the full Hamiltonian system associated to (1) with period near $2 \pi$. More specifically, these points lead to families of periodic solutions related to Hamiltonian (1) if some non-degeneracy conditions are fulfilled, as we will see in Sections 3 and 4 . For the case $h=0$ on the reduced space $\mathcal{B}(0)$ the origin in $\mathbb{R}^{9}$ is the only critical point of system (10) and it corresponds to the origin of $\mathbb{R}^{6}$ for the system related to (1).

Observation 2.2. Comparing the results obtained in the present paper for the 1:-1:1 resonance with the ones obtained in [38] for the 1:1:1 case, we notice an important difference, not only in the number, but also in the type of critical points of the reduced Hamiltonian. More precisely, in [38] we found at most thirty nine critical points on the reduced space for every positive energy level, while in this work we find at most twenty six critical points for every positive energy level and thirteen critical points for every negative energy level. 
Observation 2.3. When $\beta=0$, Hamiltonian (1) is separable, thus trivially integrable.

\section{Symplectic coordinates on the Reduced Space $\mathcal{B}(h)$}

We now define suitable variables in the neighbourhood of the critical points of propositions 2.1 and 2.2. Specifically, we consider the set of symplectic coordinates $\left(L, Q_{1}, Q_{2}, \ell, P_{1}, P_{2}\right)$ defined through the transformation $T_{1}: \Omega_{1} \rightarrow \mathbb{R}^{6}$ given by

$$
\begin{array}{ll}
x=\sqrt{2 L+Q_{1}^{2}+P_{1}^{2}-\left(Q_{2}^{2}+P_{2}^{2}\right)} \cos \ell, & X=\sqrt{2 L+Q_{1}^{2}+P_{1}^{2}-\left(Q_{2}^{2}+P_{2}^{2}\right)} \sin \ell, \\
y=Q_{1} \cos \ell+P_{1} \sin \ell, & Y=P_{1} \cos \ell-Q_{1} \sin \ell, \\
z=Q_{2} \cos \ell-P_{2} \sin \ell, & Z=P_{2} \cos \ell+Q_{2} \sin \ell,
\end{array}
$$

where

$$
\Omega_{1}=\left\{\left(L, Q_{1}, Q_{2}, \ell, P_{1}, P_{2}\right): 2 L+Q_{1}^{2}+P_{1}^{2}-\left(Q_{2}^{2}+P_{2}^{2}\right)>0,0 \leq \ell<2 \pi\right\} .
$$

The change (11) is the particularisation for the 1:-1:1 resonance of the local symplectic maps for resonant Hamiltonian systems with $n$ degrees of freedom constructed in [34], see also [33].

The coordinates $\left(L, Q_{1}, Q_{2}, \ell, P_{1}, P_{2}\right)$ are well defined for the critical points such that $\pi_{1}>0$, that is, outside the plane $O x X$. To deal with the critical points such that $\pi_{1}=0$ we introduce again symplectic coordinates $\left(L, Q_{1}, Q_{2}, \ell, P_{1}, P_{2}\right)$ through the transformations $T_{2}: \Omega_{2} \rightarrow \mathbb{R}^{6}$ and $T_{3}: \Omega_{3} \rightarrow \mathbb{R}^{6}$ defined by

$$
\begin{array}{ll}
x=Q_{1} \cos \ell-P_{1} \sin \ell, & X=P_{1} \cos \ell+Q_{1} \sin \ell, \\
y=\sqrt{-2 L+Q_{1}^{2}+P_{1}^{2}+Q_{2}^{2}+P_{2}^{2}} \cos \ell, & Y=-\sqrt{-2 L+Q_{1}^{2}+P_{1}^{2}+Q_{2}^{2}+P_{2}^{2}} \sin \ell, \\
z=Q_{2} \cos \ell-P_{2} \sin \ell, & Z=P_{2} \cos \ell+Q_{2} \sin \ell,
\end{array}
$$

and

$$
\begin{array}{ll}
x=Q_{1} \cos \ell-P_{1} \sin \ell, & X=P_{1} \cos \ell+Q_{1} \sin \ell, \\
y=Q_{2} \cos \ell+P_{2} \sin \ell, & Y=P_{2} \cos \ell-Q_{2} \sin \ell, \\
z=\sqrt{2 L-\left(Q_{1}^{2}+P_{1}^{2}\right)+Q_{2}^{2}+P_{2}^{2}} \cos \ell, & Z=\sqrt{2 L-\left(Q_{1}^{2}+P_{1}^{2}\right)+Q_{2}^{2}+P_{2}^{2}} \sin \ell,
\end{array}
$$

with

$$
\Omega_{2}=\left\{\left(L, Q_{1}, Q_{2}, \ell, P_{1}, P_{2}\right):-2 L+Q_{1}^{2}+P_{1}^{2}+Q_{2}^{2}+P_{2}^{2}>0,0 \leq \ell<2 \pi\right\}
$$

and

$$
\Omega_{3}=\left\{\left(L, Q_{1}, Q_{2}, \ell, P_{1}, P_{2}\right): 2 L-\left(Q_{1}^{2}+P_{1}^{2}\right)+Q_{2}^{2}+P_{2}^{2}>0,0 \leq \ell<2 \pi\right\} .
$$

More details on how to build these maps appear in [4]. The coordinate $\ell$ is an angle whereas $L$ corresponds to its conjugate action. Thus $\left(L, Q_{1}, Q_{2}, \ell, P_{1}, P_{2}\right)$ is a mixture of an action-angle pair and rectangular coordinates. Note that in $\Omega_{2}$, the action $L$ has to be strictly negative whereas for $\Omega_{1}, \Omega_{3}$ it can be either positive or negative.

In the coordinates introduced through the transformations $T_{1}, T_{2}$ and $T_{3}$, Hamiltonian (1) takes the form

$$
\mathcal{H}_{\varepsilon}=L+\varepsilon^{2} H_{2}\left(L, Q_{1}, Q_{2}, \ell, P_{1}, P_{2}\right)
$$

and the normalised Hamiltonian (3) reads

$$
\mathcal{H}_{\varepsilon}=L+\varepsilon^{2} \mathcal{H}_{2}\left(L, Q_{1}, Q_{2}, P_{1}, P_{2}\right)+O\left(\varepsilon^{4}\right),
$$

where $\mathcal{H}_{2}$ in each case is given explicitly by

$$
\begin{aligned}
& \mathcal{H}_{2}= \frac{3}{2} \alpha L^{2}+\frac{3}{8}(2 \alpha+\beta)\left[Q_{1}^{2}\left(2 L+Q_{1}^{2}+P_{1}^{2}-P_{2}^{2}\right)-2 Q_{1} Q_{2} P_{1} P_{2}+P_{1}^{2} P_{2}^{2}\right]+ \\
& \frac{1}{8}(6 \alpha+\beta)\left[P_{1}^{2}\left(2 L+Q_{1}^{2}+P_{1}^{2}-P_{2}^{2}\right)+2 Q_{1} Q_{2} P_{1} P_{2}+Q_{1}^{2} P_{2}^{2}\right]+ \\
& \frac{1}{8}\left(2 L+Q_{1}^{2}+P_{1}^{2}-Q_{2}^{2}-P_{2}^{2}\right)\left[3(\beta-2 \alpha) Q_{2}^{2}+(\beta-6 \alpha) P_{2}^{2}\right], \\
& 7
\end{aligned}
$$


(17)

$$
\begin{aligned}
\mathcal{H}_{2}= & \frac{3}{2} \alpha L^{2}+\frac{3}{8}(2 \alpha+\beta)\left(-2 L+Q_{1}^{2}+P_{1}^{2}+Q_{2}^{2}+P_{2}^{2}\right)\left(Q_{1}^{2}+Q_{2}^{2}\right)+\frac{3}{8}(\beta-2 \alpha)\left(Q_{1} Q_{2}+P_{1} P_{2}\right)^{2}+ \\
& \frac{1}{8}(6 \alpha+\beta)\left(-2 L+Q_{1}^{2}+P_{1}^{2}+Q_{2}^{2}+P_{2}^{2}\right)\left(P_{1}^{2}+P_{2}^{2}\right)+\frac{1}{8}(\beta-6 \alpha)\left(Q_{1} P_{2}-Q_{2} P_{1}\right)^{2},
\end{aligned}
$$

and

$$
\begin{aligned}
\mathcal{H}_{2}= & \frac{3}{2} \alpha L^{2}+\frac{3}{8}(2 \alpha+\beta)\left[Q_{2}^{2}\left(2 L-P_{1}^{2}+Q_{2}^{2}+P_{2}^{2}\right)-2 Q_{1} Q_{2} P_{1} P_{2}+P_{1}^{2} P_{2}^{2}\right]+ \\
& \frac{1}{8}(6 \alpha+\beta)\left[P_{2}^{2}\left(2 L-P_{1}^{2}+Q_{2}^{2}+P_{2}^{2}\right)+2 Q_{1} Q_{2} P_{1} P_{2}+Q_{2}^{2} P_{1}^{2}\right]+ \\
& \frac{1}{8}\left(2 L-Q_{1}^{2}-P_{1}^{2}+Q_{2}^{2}+P_{2}^{2}\right)\left[3(\beta-2 \alpha) Q_{1}^{2}+(\beta-6 \alpha) P_{1}^{2}\right],
\end{aligned}
$$

respectively.

We point out that in the case of the 1:1:1 resonance treated in [38] we only used one representation because Hamiltonian (14) has exactly the same expression for any transformation. Therefore the analysis in the 1:-1:1 case is more elaborated.

It is noticeable that in the three situations $\mathcal{H}_{2}$ is independent of $\ell$, thus the application of the transformations (11), (12) and (13) to the normal form Hamiltonian (3) produces the effect of averaging $\mathrm{H}_{2}$ with respect to the angle coordinate $\ell$. This is an expected fact that puts in emphasis the relationship between averaging and normal form theories. Applying the definition of $T_{1}, T_{2}$ or $T_{3}$ and the relations (4), we obtain

$$
\begin{aligned}
& Q_{1}=\frac{\pi_{4}}{\sqrt{\pi_{1}}}, \quad Q_{2}=\frac{\pi_{5}}{\sqrt{\pi_{1}}}, \quad P_{1}=\frac{\pi_{7}}{\sqrt{\pi_{1}}}, \quad P_{2}=\frac{\pi_{8}}{\sqrt{\pi_{1}}}, \\
& Q_{1}=\frac{\pi_{4}}{\sqrt{\pi_{2}}}, \quad Q_{2}=\frac{\pi_{6}}{\sqrt{\pi_{2}}}, \quad P_{1}=\frac{\pi_{7}}{\sqrt{\pi_{2}}}, \quad P_{2}=\frac{\pi_{9}}{\sqrt{\pi_{2}}},
\end{aligned}
$$

and

$$
Q_{1}=\frac{\pi_{5}}{\sqrt{\pi_{3}}}, \quad Q_{2}=\frac{\pi_{6}}{\sqrt{\pi_{3}}}, \quad P_{1}=-\frac{\pi_{8}}{\sqrt{\pi_{3}}}, \quad P_{2}=\frac{\pi_{9}}{\sqrt{\pi_{3}}}
$$

respectively. Thus, the maps $\psi_{1}, \psi_{2}, \psi_{3}$ with $\psi_{j}: U_{j} \rightarrow \mathbb{R}^{4}, \psi_{j}(\pi)=\left(Q_{1}, Q_{2}, P_{1}, P_{2}\right)$ defined by the equations (19), (20) and (21), respectively, are local charts for the reduced space $\mathcal{B}(h)$ for $h \neq 0$, where $U_{j}=\left\{\pi=\left(\pi_{1}, \ldots, \pi_{9}\right) \in \mathcal{B}(h): \pi_{j} \geq 0\right\}$ for $j=1,2,3$. More precisely, we arrive at the following result.

Proposition 3.1. The set $\mathcal{A}=\left\{\left(U_{1}, \psi_{1}\right),\left(U_{2}, \psi_{2}\right),\left(U_{3}, \psi_{3}\right)\right\}$ is an atlas for the reduced space $\mathcal{B}(h)$ when $h \neq 0$.

The critical points of Tables 6 and 7 in Appendix B are identified with points of $\mathbb{R}^{4}$ by means of the atlas $\mathcal{A}$. These points are shown in the coordinates $\left(Q_{1}, Q_{2}, P_{1}, P_{2}\right)$ in Appendix B in Tables 8 , 9 and 10 for $h<0$, and 11, 12 and 13 for $h>0$. Finally, applying the transformations $T_{1}, T_{2}$ and $T_{3}$, the Hamiltonians on the reduced space $\mathcal{B}(h)$ in the coordinates $\left(Q_{1}, Q_{2}, P_{1}, P_{2}\right)$ are obtained by replacing $L$ by $h \neq 0$ in (16), (17) and (18) and removing the constant term $\frac{3}{2} \alpha h^{2}$. 
By convenience we define the sets

$$
\begin{aligned}
\mathcal{R}_{1} & =\left\{O_{1}^{-}\right\} \\
\mathcal{R}_{2} & =\left\{O_{2}^{-}, O_{3}^{-}, O_{4}^{-}, O_{5}^{-}\right\} \\
\mathcal{R}_{3} & =\left\{O_{6}^{-}, O_{7}^{-}, O_{8}^{-}, O_{9}^{-}\right\} \\
\mathcal{R}_{4} & =\left\{O_{10}^{-}, O_{11}^{-}, O_{12}^{-}, O_{13}^{-}, O_{14}^{-}, O_{15}^{-}, O_{16}^{-}, O_{17}^{-}\right\} \\
\mathcal{R}_{5} & =\cup_{j=1}^{3} \mathcal{R}_{5 j}, \text { where } \mathcal{R}_{51}=\left\{O_{1}^{+}, O_{2}^{+}\right\}, \mathcal{R}_{52}=\left\{O_{3}^{+}, O_{4}^{+}\right\}, \mathcal{R}_{53}=\left\{O_{5}^{+}, O_{6}^{+}\right\} \\
\mathcal{R}_{6} & =\left\{O_{7}^{+}, O_{8}^{+}, O_{9}^{+}, O_{10}^{+}\right\} \\
\mathcal{R}_{7} & =\left\{O_{11}^{+}, O_{12}^{+}, O_{13}^{+}, O_{14}^{+}\right\} \\
\mathcal{R}_{81} & =\left\{O_{15}^{+}, O_{16}^{+}, O_{17}^{+}, O_{18}^{+}, O_{19}^{+}, O_{20}^{+}, O_{21}^{+}, O_{22}^{+}\right\} \\
\mathcal{R}_{82} & =\left\{O_{23}^{+}, O_{24}^{+}, O_{25}^{+}, O_{26}^{+}, O_{27}^{+}, O_{28}^{+}, O_{29}^{+}, O_{30}^{+}\right\}
\end{aligned}
$$

We also denote $\mathcal{R}_{8}=\mathcal{R}_{81} \cup \mathcal{R}_{82}, \mathcal{R}^{-}=\mathcal{R}_{1} \cup \mathcal{R}_{2} \cup \mathcal{R}_{3} \cup \mathcal{R}_{4}$, and $\mathcal{R}^{+}=\mathcal{R}_{5} \cup \mathcal{R}_{6} \cup \mathcal{R}_{7} \cup \mathcal{R}_{8}$.

\section{Periodic solutions and linear stability}

In what follows we denote by

$$
p(t, \varepsilon)=(x(t, \varepsilon), y(t, \varepsilon), z(t, \varepsilon), X(t, \varepsilon), Y(t, \varepsilon), Z(t, \varepsilon))
$$

a solution of system $(1)$ and by $p^{*} \in \mathcal{N}_{0}(h)$ the periodic solution associated to the critical point $\bar{p} \in \mathcal{B}(h)$, that is, $p^{*}=\rho_{\pi}^{-1}(\bar{p})$.

Our main results on the periodic solutions of the Hamiltonian system related to (1) are the following.

Theorem 4.1. When $h<0$, for the Hamiltonian system associated to (1) the following statements hold:

(i) For $\delta \in D_{1} \backslash\{-3,-1\}$ there is one $T(\varepsilon)$-periodic solution $p(t, \varepsilon)$ such that $p(t, 0)=p^{*}$ and $T(0)=2 \pi$, where $\bar{p} \in \mathcal{R}_{1}$.

(ii) If $\delta \in D_{2}$ or $\delta \in D_{3}$ there are five $T(\varepsilon)$-periodic solutions $p(t, \varepsilon)$ such that $p(t, 0)=p^{*}$ and $T(0)=2 \pi$, where $\bar{p} \in \mathcal{R}_{2}$ or $\bar{p} \in \mathcal{R}_{3}$, respectively.

(iii) If $\delta \in D_{4}$ there are thirteen $T(\varepsilon)$-periodic solutions $p(t, \varepsilon)$ such that $p(t, 0)=p^{*}$ and $T(0)=$ $2 \pi$, where $\bar{p} \in \mathcal{R}_{4}$.

(iv) When $\beta=0$ there is only one periodic solution with $\rho_{\pi}\left(p^{*}\right)=\bar{p} \in \mathcal{R}_{1}$ provided $\alpha \neq 0$.

The periodic solution $p(t, \varepsilon)$ of $(i)$ is of rectilinear type in the Oy axis. The same is true for $p(t, \varepsilon)$ in $(i v)$. The rest of solutions are elliptic inclined periodic motions.

The periods of the periodic solutions are given by $T(\varepsilon)=2 \pi\left(1-\varepsilon^{2} T^{*}\right)+O\left(\varepsilon^{4}\right)$, where the values of the corrections $T^{*}$ appear in Table 2.

Proof. Using the coordinates $\left(Q_{1}, Q_{2}, P_{1}, P_{2}\right)$ introduced through the relations $(20)$ the truncated Hamiltonian on the reduced space $\mathcal{B}(h)$ is given by

$$
\begin{aligned}
\overline{\mathcal{H}}= & \frac{1}{8} \beta(\delta+3)\left(-2 h+Q_{1}^{2}+P_{1}^{2}+Q_{2}^{2}+P_{2}^{2}\right)\left(Q_{1}^{2}+Q_{2}^{2}\right)-\frac{1}{8} \beta(\delta-3)\left(Q_{1} Q_{2}+P_{1} P_{2}\right)^{2}+ \\
& \frac{1}{8} \beta(\delta+1)\left(-2 h+Q_{1}^{2}+P_{1}^{2}+Q_{2}^{2}+P_{2}^{2}\right)\left(P_{1}^{2}+P_{2}^{2}\right)-\frac{1}{8} \beta(\delta-1)\left(Q_{1} P_{2}-Q_{2} P_{1}\right)^{2}
\end{aligned}
$$




\begin{tabular}{|c|c|c|}
\hline Set of critical points & $\operatorname{det}\left(D^{2} \overline{\mathcal{H}}\right)$ & $T^{*}$ \\
\hline $\mathcal{R}_{1}(\beta \neq 0)$ & $\frac{1}{16} h^{4} \beta^{4}(\delta+1)^{2}(\delta+3)^{2}$ & $\frac{1}{2} h \beta \delta$ \\
$\mathcal{R}_{1}(\beta=0)$ & $81 h^{4} \alpha^{4}$ & $3 h \alpha$ \\
$\mathcal{R}_{2}$ & $-\frac{h^{4} \beta^{4}(\delta-3)^{2}(\delta+3)^{2}(\delta+9)}{27(\delta+5)^{3}}$ & $\frac{h \beta(\delta-3)(\delta+6)}{6(\delta+5)}$ \\
$\mathcal{R}_{3}$ & $\frac{h^{4} \beta^{4}(\delta-3)(\delta+1)^{2}(\delta+5)}{(3 \delta+7)^{2}}$ & $\frac{h \beta\left(\delta^{2}+3 \delta-2\right)}{2(3 \delta+7)}$ \\
$\mathcal{R}_{4}$ & $-\frac{h^{4} \beta^{4}(\delta-1)(\delta-3)^{2}(\delta+1)^{2}(\delta+3)(\delta+5)^{2}}{\left(3 \delta^{2}+6 \delta-13\right)^{3}}$ & $\frac{h \beta(\delta-3)\left(\delta^{2}+3 \delta-2\right)}{2\left(3 \delta^{2}+6 \delta-13\right)}$ \\
\hline
\end{tabular}

TABle 2. Determinant of the Hessian matrix at the critical points of $\overline{\mathcal{H}}$ for $h<0$ and corrections to the periods of the periodic solutions.

and the equations of motion related to this Hamiltonian are:

$(23)$

$$
\begin{aligned}
& \dot{Q}_{1}=\frac{\beta}{4}\left[2 Q_{1} Q_{2} P_{2}-2(\delta+1) P_{1}\left(h-P_{1}^{2}\right)+2(\delta+2) Q_{1}^{2} P_{1}+(\delta+5) P_{1}\left(Q_{2}^{2}+P_{2}^{2}\right)\right], \\
& \dot{Q}_{2}=\frac{\beta}{4}\left[2 Q_{1} Q_{2} P_{1}-2(\delta+1) P_{2}\left(h-P_{2}^{2}\right)+2(\delta+2) Q_{2}^{2} P_{2}+(\delta+5) P_{2}\left(Q_{1}^{2}+P_{1}^{2}\right)\right], \\
& \dot{P}_{1}=-\frac{\beta}{4}\left[2 Q_{2} P_{1} P_{2}-2(\delta+3) Q_{1}\left(h-Q_{1}^{2}\right)+2(\delta+2) Q_{1} P_{1}^{2}+(\delta+5) Q_{1} P_{2}^{2}+(\delta+9) Q_{1} Q_{2}^{2}\right], \\
& \dot{P}_{2}=-\frac{\beta}{4}\left[2 Q_{1} P_{1} P_{2}-2(\delta+3) Q_{2}\left(h-Q_{2}^{2}\right)+2(\delta+2) Q_{2} P_{2}^{2}+(\delta+5) Q_{2} P_{1}^{2}+(\delta+9) Q_{1}^{2} Q_{2}\right] .
\end{aligned}
$$

Since the critical points $O_{1}^{-}, \ldots, O_{17}^{-}$(appearing in Tables 8, 9 and 10 in Appendix B) when expressed in the local chart $\left(U_{2}, \psi_{2}\right)$ satisfy system (23), these are equilibria for the reduced Hamiltonian (22). Moreover the determinant of the Hessian matrix $D^{2} \overline{\mathcal{H}}$ at each critical point is given in Table 2.

We have checked that the angular momentum vector in terms of the coordinates $x, y, z, X, Y$, $Z$, i.e. $(x, y, z) \times(X, Y, Z)$, becomes zero only for the equilibrium $O_{1}^{-}$, which is the one associated to the periodic solution of $(i)$ or of $(i v)$ when $\beta=0$.

The periods are computed from Hamiltonian (15) as follows. We make $\dot{\ell}=\partial \mathcal{H}_{\varepsilon} / \partial L$ and replace $Q$ and $P$ at the different values $\bar{p}$ in $\mathcal{R}_{1}, \mathcal{R}_{2}, \mathcal{R}_{3}$ and $\mathcal{R}_{4}$. Then we obtain $T(\varepsilon)$ from $\dot{\ell}=2 \pi / T(\varepsilon)$, expanding the result in powers of $\varepsilon$.

By virtue of Theorem A.2, a critical point will give rise to a family of periodic solutions provided $\operatorname{det}\left(D^{2} \overline{\mathcal{H}}\right) \neq 0$ and $\overline{\mathcal{H}}$ is a Morse function. In consequence, from Table 2 the proof of the theorem follows.

Theorem 4.2. When $h>0$, for the system associated to Hamiltonian (1) the following statements hold:

(i) For $\delta \in D_{5} \backslash\{-1,1,3\}$ there are six $T(\varepsilon)$-periodic solutions $p(t, \varepsilon)$, such that $p(t, 0)=p^{*}$ and $T(0)=2 \pi$, where $\bar{p} \in \mathcal{R}_{5}$.

(ii) If $\delta \in D_{6}$ there are ten $T(\varepsilon)$-periodic solutions $p(t, \varepsilon)$, such that $p(t, 0)=p^{*}$ and $T(0)=2 \pi$, where $\bar{p} \in \mathcal{R}_{5} \cup \mathcal{R}_{6}$.

(iii) If $\delta \in D_{7} \backslash\{-3\}$ there are eighteen $T(\varepsilon)$-periodic solutions $p(t, \varepsilon)$, such that $p(t, 0)=p^{*}$ and $T(0)=2 \pi$, where $\bar{p} \in \mathcal{R}_{5} \cup \mathcal{R}_{7} \cup \mathcal{R}_{82}$. 
(iv) If $\delta \in D_{8}$ there are twenty-six $T(\varepsilon)$-periodic solutions $p(t, \varepsilon)$, such that $p(t, 0)=p^{*}$ and $T(0)=2 \pi$, where $\bar{p} \in \mathcal{R}_{5} \cup \mathcal{R}_{7} \cup \mathcal{R}_{8}$.

(v) When $\beta=0$, there are two periodic solutions with $\rho_{\pi}\left(p^{*}\right)=\bar{p} \in \mathcal{R}_{51}$ provided $\alpha \neq 0$.

The periodic solutions $p(t, \varepsilon)$ related to $\bar{p} \in \mathcal{R}_{51}$ and $\bar{p} \in \mathcal{R}_{53}$ are near rectilinear whereas when $\bar{p} \in \mathcal{R}_{52}$ they are near circular. The periodic solutions associated with $\bar{p} \in \mathcal{R}_{82}$ are also near circular. The rest of solutions represent elliptic inclined periodic motions.

The periods of the periodic solutions are given by $T(\varepsilon)=2 \pi\left(1-\varepsilon^{2} T^{*}\right)+O\left(\varepsilon^{4}\right)$, where the values of the corrections $T^{*}$ appear in Table 3.

\begin{tabular}{|c|c|c|}
\hline Set of critical points & $\operatorname{det}\left(D^{2} \overline{\mathcal{H}}\right)$ & $T^{*}$ \\
\hline $\mathcal{R}_{51}(\beta \neq 0)$ & $\frac{1}{16} h^{4} \beta^{4}\left(\delta^{2}-1\right)\left(\delta^{2}-9\right)$ & $\frac{1}{2} h \beta \delta$ \\
$\mathcal{R}_{51}(\beta=0)$ & $81 h^{4} \alpha^{4}$ & $3 h \alpha$ \\
$\mathcal{R}_{52}$ & $\frac{1}{32} h^{4} \beta^{4}(\delta-1)(\delta+5)^{2}$ & $\frac{1}{4} h \beta(\delta+1)$ \\
$\mathcal{R}_{53}$ & $-\frac{1}{32} h^{4} \beta^{4}(\delta-3)(\delta+5)(\delta+9)$ & $\frac{1}{4} h \beta(\delta+3)$ \\
$\mathcal{R}_{6}$ & $-\frac{h^{4} \beta^{4}(\delta-3)^{2}(\delta+3)^{2}(\delta+9)}{27(\delta+5)^{3}}$ & $\frac{h \beta(\delta-3)(\delta+6)}{6(\delta+5)}$ \\
$\mathcal{R}_{7}$ & $\frac{h^{4} \beta^{4}(\delta-3)(\delta+1)^{2}(\delta+5)}{(3 \delta+7)^{2}}$ & $\frac{h \beta\left(\delta^{2}+3 \delta-2\right)}{2(3 \delta+7)}$ \\
$\mathcal{R}_{81}$ & $-\frac{h^{4} \beta^{4}(\delta-3)^{2}(\delta-1)(\delta+1)^{2}(\delta+3)(\delta+5)^{2}}{\left(3 \delta^{2}+6 \delta-13\right)^{3}}$ & $\frac{h \beta(\delta-3)\left(\delta^{2}+3 \delta-2\right)}{2\left(3 \delta^{2}+6 \delta-13\right)}$ \\
$\mathcal{R}_{82}$ & $\frac{h^{4} \beta^{4}(\delta-3)^{2}(\delta+5)^{2}}{4(3 \delta+7)^{2}}$ & $\frac{h \beta\left(\delta^{2}-9\right)}{2(3 \delta+7)}$ \\
\hline
\end{tabular}

TABle 3. Determinant of the Hessian matrix at the critical points of $\overline{\mathcal{H}}$ for $h>0$ and corrections to the periods of the periodic solutions.

Proof. In the coordinates $\left(Q_{1}, Q_{2}, P_{1}, P_{2}\right)$ defined in (19) the reduced Hamiltonian on $\mathcal{B}(h)$ assumes the form

$$
\begin{aligned}
\overline{\mathcal{H}}= & \frac{1}{8} \beta(\delta+3)\left[Q_{1}^{2}\left(2 h+Q_{1}^{2}+P_{1}^{2}-P_{2}^{2}\right)-2 Q_{1} Q_{2} P_{1} P_{2}+P_{1}^{2} P_{2}^{2}\right]+ \\
& \frac{1}{8} \beta(\delta+1)\left[P_{1}^{2}\left(2 h+Q_{1}^{2}+P_{1}^{2}-P_{2}^{2}\right)+2 Q_{1} Q_{2} P_{1} P_{2}+Q_{1}^{2} P_{2}^{2}\right]- \\
& \frac{1}{8} \beta\left(2 h+Q_{1}^{2}+P_{1}^{2}-Q_{2}^{2}-P_{2}^{2}\right)\left[(\delta-3) Q_{2}^{2}+(\delta-1) P_{2}^{2}\right],
\end{aligned}
$$


and the related equations of motion are:

$(25)$

$$
\begin{aligned}
& \dot{Q}_{1}=\frac{\beta}{4}\left[-2 Q_{1} Q_{2} P_{2}+2(\delta+1) P_{1}\left(h+P_{1}^{2}\right)-(\delta-3) P_{1}\left(Q_{2}^{2}+P_{2}^{2}\right)+2(\delta+2) Q_{1}^{2} P_{1}\right], \\
& \dot{Q}_{2}=\frac{\beta}{4}\left[-2 Q_{1} Q_{2} P_{1}-(\delta-3) P_{1}^{2} P_{2}-2(\delta-1) P_{2}\left(h-P_{2}^{2}\right)+2(\delta-2) Q_{2}^{2} P_{2}-(\delta+1) Q_{1}^{2} P_{2}\right], \\
& \dot{P}_{1}=\frac{\beta}{4}\left[2 Q_{2} P_{1} P_{2}-2(\delta+2) Q_{1} P_{1}^{2}-2(\delta+3) Q_{1}\left(h+Q_{1}^{2}\right)+(\delta-3) Q_{1} Q_{2}^{2}+(\delta+1) Q_{1} P_{2}^{2}\right], \\
& \dot{P}_{2}=\frac{\beta}{4}\left[2 Q_{1} P_{1} P_{2}+(\delta-3) Q_{2}\left(2 h+Q_{1}^{2}-2 Q_{2}^{2}+P_{1}^{2}\right)-2(\delta-2) Q_{2} P_{2}^{2}\right] .
\end{aligned}
$$

The critical points $O_{1}^{+}, O_{3}^{+}, \ldots, O_{30}^{+}$appearing in Tables 11, 12 and 13 in Appendix B in the local chart $\left(U_{1}, \psi_{1}\right)$ satisfy system $(25)$. So, they are equilibrium points for the reduced Hamiltonian (24). The determinant of the Hessian matrix $D^{2} \overline{\mathcal{H}}$ evaluated at each critical point is given in Table 3. Next, as it was done in the previous theorem, one applies Theorem A.2 and takes into account that the previous Hessian matrices are non-singular and that $\overline{\mathcal{H}}$ is a Morse function.

For the analysis of the equilibrium point $O_{2}^{+}$we consider the coordinates $\left(Q_{1}, Q_{2}, P_{1}, P_{2}\right)$ defined in (21) and proceed as before but with Hamiltonian (18).

Computing the angular momentum vector in terms of the coordinates $x, y, z, X, Y, Z$ we get that it is identically zero only for the equilibria $\bar{p} \in \mathcal{R}_{51}$ or $\bar{p} \in \mathcal{R}_{53}$, thus the corresponding periodic solutions are near rectilinear. More specifically, the periodic solution corresponding to the critical point $O_{1}^{+}$is in the $O x$ axis, the one corresponding to the critical point $O_{2}^{+}$is in the $O z$ axis while the ones corresponding to $O_{5}^{+}, O_{6}^{+}$lie in the plane $O x z$. Besides, we have calculated the expressions $x^{2}+y^{2}+z^{2}$ and $X^{2}+Y^{2}+Z^{2}$ in terms of the local variables $Q_{i}, P_{i}, \ell, L$ for the different equilibria, verifying that they are constant only when $\bar{p} \in \mathcal{R}_{52}$ and $\bar{p} \in \mathcal{R}_{82}$. As a consequence the corresponding periodic solutions $p(t, \varepsilon)$ are of circular type. They lie in the plane $O x z$.

The change in the number of periodic solutions of Hamiltonian (1) and their stability as long as the parameters $\alpha$ and $\beta$ vary is related to the appearance of parametric bifurcation lines, see Figs. 1 and 2. The bifurcations of periodic solutions are analysed in the reduced space $\mathcal{B}(h)$ or even better, when possible, in spaces of lower dimension, reconstructing the flow of the full system as a final step. Some studies have been established in some cases, see for instance [31, 33]. For systems with three or more degrees of freedom one should consult the work of Hanßmann and van der Meer [19], and also the papers [13, 11, 10, 2, 28, 4]. For a rather complete treatment regarding bifurcations of periodic solutions and invariant tori the reader should look up the book [18].

Observation 4.1. As a difference with respect to the perturbed Hamiltonian in 1:1:1 resonance treated in [38], here we have a greater number of bifurcation curves due to the restrictions given in (5) together with the sign of each nonzero energy level $h$.

The upcoming results provide information on the linear stability of the periodic solutions established in Theorems 4.1 and 4.2 .

Theorem 4.3. Under the assumptions of Theorem 4.1, the following statements hold:

(i) The near rectilinear periodic solution generated by the critical point in $\mathcal{R}_{1}$ is linearly stable when $\delta \in(-\infty,-3) \cup(-1,+\infty)$ and is unstable in the Lyapunov sense when $\delta \in(-3,-1)$, whereas the periodic solutions generated by the critical points in $\mathcal{R}_{2} \cup \mathcal{R}_{3} \cup \mathcal{R}_{4}$ are unstable in the Lyapunov sense. When $\beta=0$ the periodic solution related to the set $\mathcal{R}_{1}$ is linearly stable. 


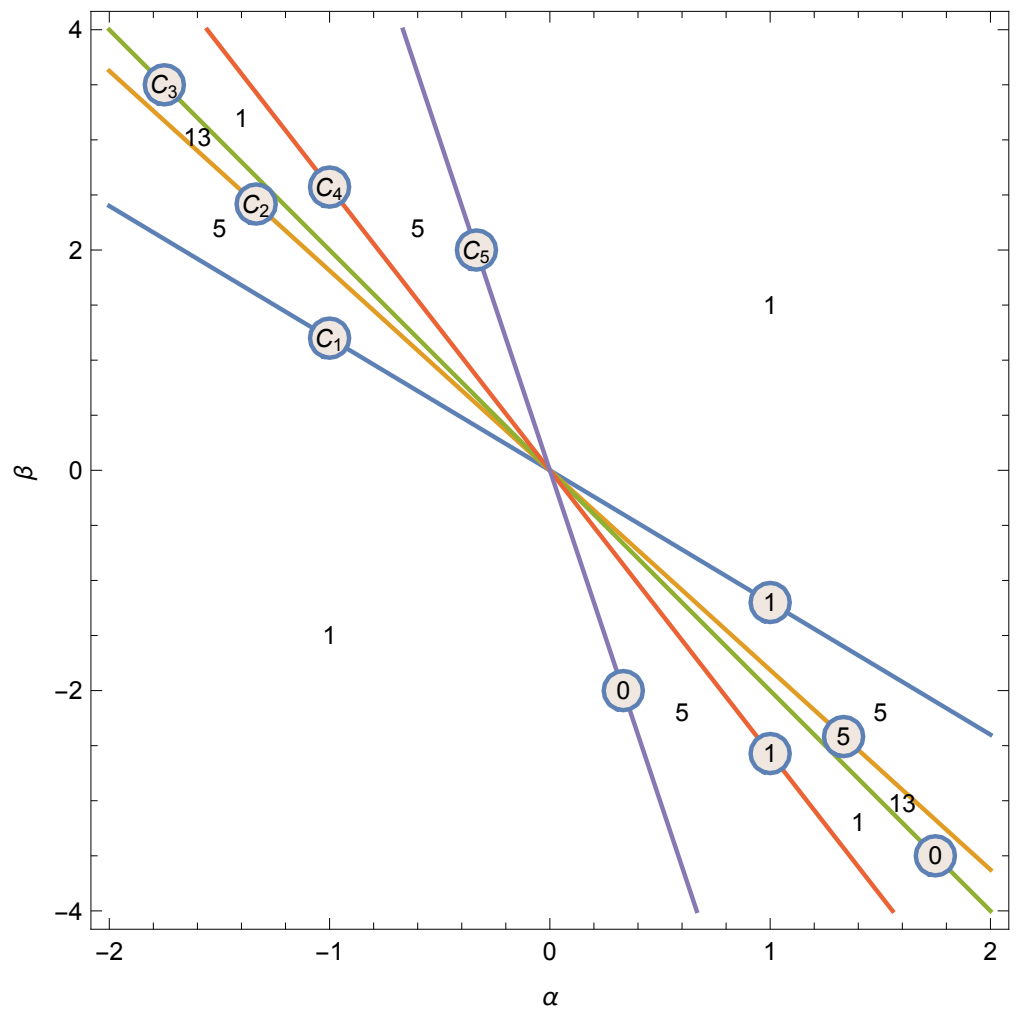

Figure 1. Bifurcation diagram of the periodic solutions in the plane of parameters $\alpha-\beta$ for $h<0$. The lines $C_{1}$ and $C_{2}$ correspond to the equations $6 \alpha+5 \beta=0$ $(\delta=-5)$ and $6 \alpha+(3+4 \sqrt{3}) \beta / \sqrt{3}=0(\delta=-(3+4 \sqrt{3}) / \sqrt{3})$, respectively. The lines $C_{3}$ and $C_{4}$ correspond to the equations $2 \alpha+\beta=0(\delta=-3)$ and $6 \alpha+7 \beta / 3=0$ $(\delta=-7 / 3)$, respectively. The line $C_{5}$ corresponds to the equation $6 \alpha+\beta=0$ $(\delta=-1)$. The number of families of periodic solutions is indicated in each region/line.

(ii) The characteristic multipliers of the periodic solutions mentioned in (i) are given by $1,1,1 \pm$ $\pi \varepsilon^{2} \lambda_{1}+O\left(\varepsilon^{4}\right), 1 \pm \pi \varepsilon^{2} \lambda_{2}+O\left(\varepsilon^{4}\right)$, where $\lambda_{1}$ and $\lambda_{2}$ are the eigenvalues of matrix $A=\mathbb{J} D^{2} \overline{\mathcal{H}}$ (see Table 4).

Proof. The linear stability or instability of the periodic solutions in Theorem 4.1 is determined by the strong stability or instability of the corresponding critical points in the set $\mathcal{R}^{-}$. For this purpose, if $\mathbb{J}$ denotes the standard skew-symmetric matrix of order four, we calculate the eigenvalues of matrix $A=\mathbb{J} D^{2} \overline{\mathcal{H}}$ for each critical point and use the notation of Theorem A.3.

(a) For the critical point in $\mathcal{R}_{1}$, the linearisation matrix is

$$
A\left(\mathcal{R}_{1}\right)=\left[\begin{array}{cccc}
0 & 0 & -\frac{1}{2} h \beta(\delta+1) & 0 \\
0 & 0 & 0 & -\frac{1}{2} h \beta(\delta+1) \\
\frac{1}{2} h \beta(\delta+3) & 0 & 0 & 0 \\
0 & \frac{1}{2} h \beta(\delta+3) & 0 & 0
\end{array}\right]
$$




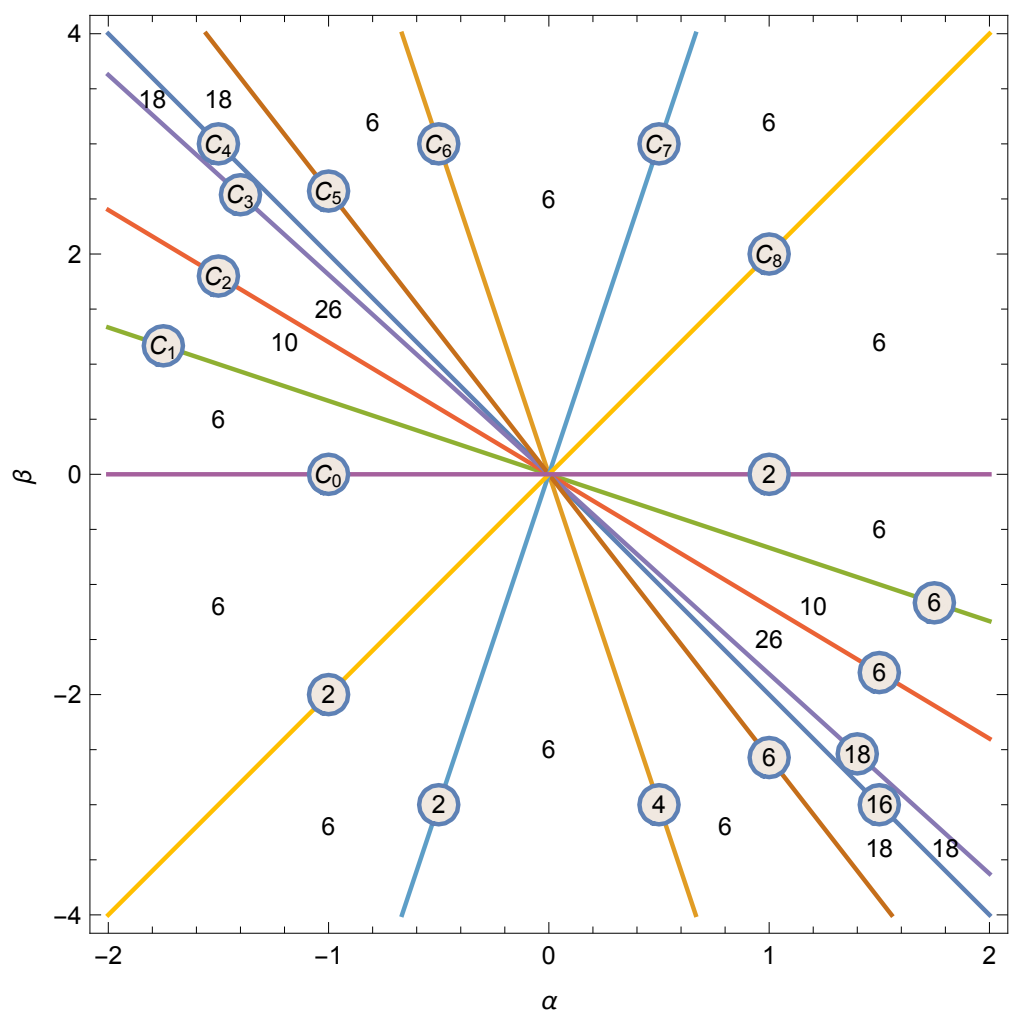

FigURE 2. Bifurcation diagram of the periodic solutions in the plane of parameters $\alpha-\beta$ for $h>0$. The lines $C_{0}$ and $C_{1}$ correspond to the equations $\beta=0$ and $6 \alpha+9 \beta=0(\delta=-9)$, respectively. The lines $C_{2}$ and $C_{3}$ correspond to the equations $6 \alpha+5 \beta=0(\delta=-5)$ and $6 \alpha+(3+4 \sqrt{3}) \beta / 3=0(\delta=-(3+4 \sqrt{3}) / 3)$, respectively. The lines $C_{4}$ and $C_{5}$ correspond to the equations $2 \alpha+\beta=0(\delta=-3)$ and $6 \alpha+$ $7 \beta / 3=0(\delta=-7 / 3)$, respectively. The lines $C_{6}$ and $C_{7}$ correspond to the equations $6 \alpha+\beta=0(\delta=-1)$ and $6 \alpha-\beta=0(\delta=1)$, respectively. The line $C_{8}$ corresponds to the equation $2 \alpha-\beta=0(\delta=3)$. The number of families of periodic solutions is indicated in each region/line.

and the characteristic polynomial is

$$
C_{\lambda}=\left[\lambda^{2}+\frac{1}{4} h^{2} \beta^{2}(\delta+1)(\delta+3)\right]^{2} .
$$

Thus, for $\delta \in(-3,-1)$ the matrix $A\left(\mathcal{R}_{1}\right)$ has eigenvalues with non-zero real part. In consequence, by means of Theorem A.3, the near rectilinear periodic solution in the $O y$ axis is unstable in the Lyapunov sense. On the other hand, for $\delta \in(-\infty,-3) \cup(-1, \infty)$, $A\left(\mathcal{R}_{1}\right)$ is diagonalisable and its eigenvalues are $\pm \alpha_{1} i$ (with multiplicity 2 ) where

$$
\alpha_{1}=\frac{1}{2} h \beta \sqrt{(\delta+1)(\delta+3)} .
$$

The quadratic Hamiltonian associated to $A\left(\mathcal{R}_{1}\right)$ in some symplectic coordinates $(q, p)$ is given by

$$
\mathcal{K}=-\frac{1}{4} h \beta\left[(\delta+3)\left(q_{1}^{2}+q_{2}^{2}\right)+(\delta+1)\left(p_{1}^{2}+p_{2}^{2}\right)\right] .
$$

The maximal real linear subspace corresponding to $\alpha_{1}$ is $V_{1}=\operatorname{Ker}\left(A-\alpha_{1} i I\right)=\mathbb{R}^{4}$ and $\mathcal{K}_{1}=\left.\mathcal{K}\right|_{V_{1}}=\mathcal{K}$ has definite sign. So, $A\left(\mathcal{R}_{1}\right)$ is strongly stable and applying Theorem A.3 it follows that the near rectilinear periodic solution in the $O y$ axis is linearly stable. 


\begin{tabular}{|c|c|}
\hline Critical point & Eigenvalues \\
\hline $\mathcal{R}_{1}(\beta \neq 0)$ & $\lambda_{1}=\lambda_{2}=\frac{h \beta}{2} \sqrt{-(\delta+1)(\delta+3)}$ \\
$\mathcal{R}_{1}(\beta=0)$ & $\lambda_{1}=\lambda_{2}=3 h \alpha i$ \\
\hline $\mathcal{R}_{2}$ & $\lambda_{1}=h \beta \sqrt{\frac{-(\delta+3)(\delta+9)}{3(\delta+5)}}, \lambda_{2}=\frac{h \beta(\delta-3) \sqrt{-(\delta+3)}}{3(\delta+5)} i$ \\
\hline $\mathcal{R}_{3}$ & $\lambda_{1}=h \beta \sqrt{\frac{(\delta+1)(\delta-3)}{3 \delta+7}}, \lambda_{2}=h \beta \sqrt{\frac{-(\delta+1)(\delta+5)}{3 \delta+7}} i$ \\
\hline $\mathcal{R}_{4}$ & $\lambda_{1}=\frac{h \beta}{3 \delta^{2}+6 \delta-13} \sqrt{-(\delta+1)\left[\Delta_{1}-2 \sqrt{(\delta-1)(\delta+3) \Delta_{2}}\right]}$ \\
& $\lambda_{2}=\frac{h \beta}{3 \delta^{2}+6 \delta-13} \sqrt{-(\delta+1)\left[\Delta_{1}+2 \sqrt{(\delta-1)(\delta+3) \Delta_{2}}\right]}$ \\
\hline
\end{tabular}

TABLE 4. Eigenvalues of the matrix $A$ for $h<0$, where $\Delta_{1}=-51+\delta(\delta+2)\left(\delta^{2}+\right.$ $2 \delta+14)$ and $\Delta_{2}=-948+\delta(\delta+2)\left[313+\delta(\delta+2)\left(\delta^{2}+2 \delta-18\right)\right]$.

(b) For the critical points of $\mathcal{R}_{2}$ the linearisation around them given by the matrix $A$ becomes

$$
A\left(\mathcal{R}_{2}\right)=\left[\begin{array}{cccc}
0 & 0 & \frac{2 h \beta}{\delta+5} & \mp \frac{h \beta(\delta+3)}{3(\delta+5)} \\
0 & 0 & \mp \frac{h \beta(\delta+3)}{3(\delta+5)} & \frac{2 h \beta}{\delta+5} \\
-\frac{2 h \beta(\delta+3)^{2}}{3(\delta+5)} & \pm \frac{h \beta(\delta+3)(\delta+9)}{3(\delta+5)} & 0 & 0 \\
\pm \frac{h \beta(\delta+3)(\delta+9)}{3(\delta+5)} & -\frac{2 h \beta(\delta+3)^{2}}{3(\delta+5)} & 0 & 0
\end{array}\right]
$$

where the upper signs are used for $O_{2}^{-}, O_{4}^{-}$and the lower ones for $O_{3}^{-}$and $O_{5}^{-}$. The characteristic polynomial in this case reads

$$
C_{\lambda}=\left[\lambda^{2}+\frac{h^{2} \beta^{2}(\delta+3)(\delta+9)}{3(\delta+5)}\right]\left[\lambda^{2}-\frac{h^{2} \beta^{2}(\delta-3)^{2}(\delta+3)}{9(\delta+5)^{2}}\right] .
$$

Thence, for $\delta \in D_{2} \cup D_{4}$ the matrix $A\left(\mathcal{R}_{2}\right)$ has two real eigenvalues $\pm \alpha_{1}$ of the form

$$
\alpha_{1}=h \beta \sqrt{\frac{-(\delta+3)(\delta+9)}{3(\delta+5)}},
$$

whereas the other two eigenvalues are purely imaginary. Therefore, by means of Theorem A.3 the periodic solutions generated by the critical points in $\mathcal{R}_{2}$ are unstable in the Lyapunov sense.

(c) The matrix $A$ evaluated at the critical points of $\mathcal{R}_{3}$ yields

$$
A\left(\mathcal{R}_{3}\right)=\left[\begin{array}{cccc}
0 & 0 & \frac{2 h \beta(\delta+1)^{2}}{3 \delta+7} & \mp \frac{h \beta(\delta+1)(\delta+5)}{3 \delta+7} \\
0 & 0 & \mp \frac{h \beta(\delta+1)(\delta+5)}{3 \delta+7} & \frac{2 h \beta(\delta+1)^{2}}{3 \delta+7} \\
\frac{2 h \beta(\delta+3)}{3 \delta+7} & \pm \frac{h \beta(\delta+1)}{3 \delta+7} & 0 & 0 \\
\pm \frac{h \beta(\delta+1)}{3 \delta+7} & \frac{2 h \beta(\delta+3)}{3 \delta+7} & 0 & 0
\end{array}\right]
$$


where the upper signs are used for $O_{6}^{-}, O_{7}^{-}$and the lower ones for $O_{8}^{-}$and $O_{9}^{-}$. The related characteristic polynomial is given by

$$
C_{\lambda}=\left[\lambda^{2}-\frac{h^{2} \beta^{2}(\delta+1)(\delta-3)}{3 \delta+7}\right]\left[\lambda^{2}-\frac{h^{2} \beta^{2}(\delta+1)(\delta+5)}{3 \delta+7}\right] .
$$

So, for $\delta \in D_{3}$, matrix $A\left(\mathcal{R}_{3}\right)$ has two real eigenvalues $\pm \alpha_{1}$ with

$$
\alpha_{1}=h \beta \sqrt{\frac{(\delta+1)(\delta-3)}{3 \delta+7}} .
$$

The other eigenvalues are purely imaginary. Thus, the periodic solutions are unstable in the Lyapunov sense.

(d) For the critical points $O_{10}^{-}, \ldots, O_{13}^{-}$in $\mathcal{R}_{4}$ the matrix is given by

$$
A\left(\mathcal{R}_{4}\right)=\left[\begin{array}{cccc}
0 & \pm \frac{h \beta(\delta+1) \sqrt{\delta^{2}-9}}{3 \delta^{2}+6 \delta-13} & \frac{2 h \beta\left(\delta^{2}+2 \delta-7\right)}{3 \delta^{2}+6 \delta-13} & 0 \\
\pm \frac{h \beta(\delta+1)(\delta+5) \sqrt{\delta^{2}-9}}{3 \delta^{2}+6 \delta-13} & 0 & 0 & \frac{2 h \beta(\delta-3)(\delta+1)(\delta+3)}{3 \delta^{2}+6 \delta-13} \\
-\frac{2 h \beta(\delta+1)^{2}(\delta+3)}{3 \delta^{2}+6 \delta-13} & 0 & 0 & \mp \frac{h \beta(\delta+1)(\delta+5) \sqrt{\delta^{2}-9}}{3 \delta^{2}+6 \delta-13} \\
0 & \frac{2 h \beta(\delta-3)}{3 \delta^{2}+6 \delta-13} & \mp \frac{h \beta(\delta+1) \sqrt{\delta^{2}-9}}{3 \delta^{2}+6 \delta-13} & 0
\end{array}\right],
$$

where the upper signs in $A\left(\mathcal{R}_{4}\right)$ correspond to $O_{10}^{-}, O_{12}^{-}$and the lower ones to $O_{11}^{-}, O_{13}^{-}$.

The linearisation matrix of the points $O_{14}^{-}, \ldots, O_{17}^{-}$is

$$
A^{\prime}\left(\mathcal{R}_{4}\right)=\left[\begin{array}{cccc}
0 & \mp \frac{h \beta(\delta+1)(\delta+5) \sqrt{\delta^{2}-9}}{3 \delta^{2}+6 \delta-13} & \frac{2 h \beta(\delta+1)\left(\delta^{2}-9\right)}{3 \delta^{2}+6 \delta-13} & 0 \\
\mp \frac{h \beta(\delta+1) \sqrt{\delta^{2}-9}}{3 \delta^{2}+6 \delta-13} & 0 & 0 & \frac{2 h \beta\left(\delta^{2}+2 \delta-7\right)}{3 \delta^{2}+6 \delta-13} \\
\frac{2 h \beta(\delta-3)}{3 \delta^{2}+6 \delta-13} & 0 & 0 & \pm \frac{h \beta(\delta+1) \sqrt{\delta^{2}-9}}{3 \delta^{2}+6 \delta-13} \\
0 & -\frac{2 h \beta(\delta+1)^{2}(\delta+3)}{3 \delta^{2}+6 \delta-13} & \pm \frac{h \beta(\delta+1)(\delta+5) \sqrt{\delta^{2}-9}}{3 \delta^{2}+6 \delta-13} & 0
\end{array}\right] .
$$

The upper signs in $A^{\prime}\left(\mathcal{R}_{4}\right)$ are related to $O_{14}^{-}, O_{16}^{-}$while the lower signs refer to $O_{15}^{-}, O_{17}^{-}$.

The characteristic polynomial for both $A\left(\mathcal{R}_{4}\right)$ and $A^{\prime}\left(\mathcal{R}_{4}\right)$ is

$$
C_{\lambda}=\lambda^{4}+\frac{2 h^{2} \beta^{2}\left(\delta^{2}-1\right)(\delta+3)\left(\delta^{2}+2 \delta+17\right)}{\left(3 \delta^{2}+6 \delta-13\right)^{2}} \lambda^{2}-\frac{h^{4} \beta^{4}(\delta-3)^{2}(\delta-1)(\delta+1)^{2}(\delta+3)(\delta+5)^{2}}{\left(3 \delta^{2}+6 \delta-13\right)^{3}} .
$$

Proceeding in a similar way to the above items we verify that for $\delta \in D_{4}, C_{\lambda}$ has complex roots with non-null real part (complex instability), hence the corresponding periodic solutions are unstable.

(e) If $\beta=0$, the matrix $A\left(\mathcal{R}_{1}\right)$ becomes

$$
A^{*}\left(\mathcal{R}_{1}\right)=\left[\begin{array}{cccc}
0 & 0 & -3 h \alpha & 0 \\
0 & 0 & 0 & -3 h \alpha \\
3 h \alpha & 0 & 0 & 0 \\
0 & 3 h \alpha & 0 & 0
\end{array}\right]
$$

from which we get strong stability for the point $O_{1}^{-}$, hence the elliptic character of the periodic solutions is readily deduced. 
The characteristic multipliers of the periodic solutions are approximated using Theorem A.2 of Appendix A, see also [30] and [50], but taking into account that we need to reach order two in $\varepsilon$ as order one is identically zero. Specifically one has to compute the eigenvalues of the matrices $A$ of the previous paragraphs (which has been done explicitly in most cases). These eigenvalues are displayed in Table 4.

Theorem 4.4. Under the assumptions of Theorem 4.2, the following statements hold:

(i) The near rectilinear periodic solutions in the principal axes generated by the critical points in $\mathcal{R}_{51}$ are linearly stable when $\delta \in(-\infty,-3) \cup(-1,1) \cup(3, \infty)$ and are unstable in the Lyapunov sense when $\delta \in(-3,-1) \cup(1,3)$.

(ii) The near circular periodic solutions generated by the critical points in $\mathcal{R}_{52}$ are linearly stable when $\delta \in(1, \infty)$ and are unstable in the Lyapunov sense when $\delta \in(-\infty,-5) \cup(-5,1)$.

(iii) The near rectilinear periodic solutions generated by the critical points in $\mathcal{R}_{53}$ are linearly stable when $\delta \in(-\infty,-9) \cup(-5,3)$ and are unstable in the Lyapunov sense when $\delta \in$ $(-9,-5) \cup(3, \infty)$.

(iv) The periodic solutions generated by the critical points in $\mathcal{R}_{6}$ are linearly stable.

(v) The periodic solutions generated by the critical points in $\mathcal{R}_{7}$ are unstable in the Lyapunov sense.

(vi) The periodic solutions generated by the critical points in $\mathcal{R}_{8}$ are unstable in the Lyapunov sense.

The characteristic multipliers of the periodic solutions are given by $1,1,1 \pm \pi \varepsilon^{2} \lambda_{1}+O\left(\varepsilon^{4}\right), 1 \pm$ $\pi \varepsilon^{2} \lambda_{2}+O\left(\varepsilon^{4}\right)$ where $\lambda_{1}$ and $\lambda_{2}$ are the eigenvalues of matrix $A=\mathbb{J} D^{2} \overline{\mathcal{H}}$ evaluated at the critical points (see Table 5).

Proof. Proceeding in a similar way to the proof of Theorem 4.3 for all critical points $O_{i}^{+}$, we conclude the statements of the theorem through the eigenvalues given in Table 5 together with their associated eigenvectors.

Observation 4.2. In the reduced space, there are one-parameter families of periodic orbits emanating from the equilibria

- $\mathcal{R}_{2} \cup \mathcal{R}_{3} \cup \mathcal{R}_{52} \cup \mathcal{R}_{53} \cup \mathcal{R}_{7} \cup \mathcal{R}_{81}$;

- $\mathcal{R}_{51}(\beta \neq 0)$ when $\delta \in(-\infty,-3)$ and $\frac{\lambda_{2}}{\lambda_{1}} \notin \mathbb{Z}^{+}$or when $\delta \in(-1,1)$ and $\left\{\frac{\lambda_{1}}{\lambda_{2}}, \frac{\lambda_{2}}{\lambda_{1}}\right\} \notin \mathbb{Z}^{+}$or when $\delta \in(3,+\infty)$ and $\frac{\lambda_{1}}{\lambda_{2}} \notin \mathbb{Z}^{+}$;

- $\mathcal{R}_{6}$ when $\frac{\lambda_{2}}{\lambda_{1}} \notin \mathbb{Z}^{+}$.

These are the Lyapunov orbits (see, for instance, [31]) in $\mathcal{B}(h)$. They would correspond to invariant $2 D$ tori in the full system.

\section{BifurCATions of SOME PERIODiC SOLUTIONS}

The analysis of the all bifurcations occurring regarding the periodic solutions established in Theorems 4.1 and 4.2 of Section 4 should be done globally, considering the number and linear stability of the periodic solutions in the different regions depending on $\beta$ and $\delta$. This would be a formidable task that is out of the scope of this paper. Here we focus on the bifurcations of the near rectilinear or near circular periodic solutions associated to the sets $\mathcal{R}_{1}$ and $\mathcal{R}_{5}$.

We want to stress the feasibility of the local symplectic variables $Q_{i} / P_{i}$ introduced through the maps (11), (12) and (13). They are indeed rather convenient coordinates to perform the symplectic transformations towards the determination of the various normal forms needed to establish the different bifurcations occurring in a system with three or more degrees of freedom.

The first result concerns the bifurcations related with the points in the set $\mathcal{R}_{5}$, that is, with periodic motions that are near rectilinear in the $O x$ and $O z$ axes. 


\begin{tabular}{|c|c|}
\hline Critical point & Eigenvalues \\
\hline $\mathcal{R}_{51}(\beta \neq 0)$ & $\lambda_{1}=\frac{h \beta}{2} \sqrt{-(\delta+1)(\delta+3)}, \lambda_{2}=\frac{h \beta}{2} \sqrt{-(\delta-3)(\delta-1)}$ \\
$\mathcal{R}_{51}(\beta=0)$ & $\lambda_{1}=\lambda_{2}=3 h \alpha i$ \\
$\mathcal{R}_{52}$ & $\lambda_{1}=h \beta \sqrt{\frac{1-\delta}{2}}, \lambda_{2}=\frac{h \beta}{4}|\delta+5| i$ \\
$\mathcal{R}_{53}$ & $\lambda_{1}=h \beta \sqrt{\frac{3-\delta}{2}} i, \lambda_{2}=\frac{h \beta}{4} \sqrt{-(\delta+5)(\delta+9)}$ \\
\hline $\mathcal{R}_{6}$ & $\lambda_{1}=h \beta \sqrt{\frac{(\delta+3)(\delta+9)}{3(\delta+5)}} i, \lambda_{2}=\frac{h \beta(\delta-3) \sqrt{-(\delta+3)}}{3(\delta+5)} i$ \\
\hline $\mathcal{R}_{7}$ & $\lambda_{1}=h \beta \sqrt{\frac{(\delta+1)(\delta+5)}{3 \delta+7}}, \lambda_{2}=h \beta \sqrt{\frac{-(\delta-3)(\delta+1)}{3 \delta+7}} i$ \\
\hline $\mathcal{R}_{81}=\frac{h \beta}{3 \delta^{2}+6 \delta-13} \sqrt{\frac{(\delta+1)\left[\Delta_{1}-2 \sqrt{(\delta-1)(\delta+3) \Delta_{2}}\right.}{2}} i$ \\
$\mathcal{R}_{82}$ & $\frac{h \beta}{3 \delta^{2}+6 \delta-13} \sqrt{\frac{(\delta+1)\left[-\Delta_{1}-2 \sqrt{(\delta-1)(\delta+3) \Delta_{2}}\right.}{2}}$ \\
$\lambda_{1}=h \beta \sqrt{\frac{-\left(\delta^{2}+5 \delta+24\right)+\sqrt{3(\delta+13)\left(2 \delta^{2}+7 \delta+9\right)}}{2(3 \delta+7)}}$ \\
$\lambda_{2}=h \beta \sqrt{\frac{-\left(\delta^{2}+5 \delta+24\right)-\sqrt{3(\delta+13)\left(2 \delta^{2}+7 \delta+9\right)}}{2(3 \delta+7)}}$
\end{tabular}

TABle 5. Eigenvalues of the matrix $A$ evaluated at the critical points for $h>0$, with $\Delta_{1}$ and $\Delta_{2}$ introduced in the caption of Table 4 . The linearisation of the critical points in $\mathcal{R}_{7}$ and $\mathcal{R}_{81}$ is of the form centre $\times$ saddle while the one of the points in $\mathcal{R}_{82}$ is of the form saddle $\times$ saddle.

Proposition 5.1. Consider a fixed value $h>0$ and take $\mathcal{H}_{0}=h$. On the reduced space $\mathcal{B}(h)$ there are periodic Hamiltonian pitchfork bifurcations of subcritical type related to the point $O_{1}^{+}$for $\delta$ near -1 and to the points $O_{5}^{+}, O_{6}^{+}$for $\delta$ near -5 . There are periodic Hamiltonian pitchfork bifurcations of supercritical type related to the point $O_{1}^{+}$for $\delta$ near -3 and near 1 and to the points $O_{5}^{+}, O_{6}^{+}$for $\delta$ near -9 .

Proof. In the supercritical case there is a periodic solution that loses its stability, giving rise to an additional pair of linearly stable periodic solutions whereas in the subcritical case the stability character of the periodic solution is lost as a pair of unstable periodic solutions shrinks down. See the details on pitchfork bifurcations involving periodic solutions in [18].

The proposition is proved for $O_{1}^{+}$with $\delta \approx-1$ while the other cases are analogous. We use the variables $Q / P$ defined in (11) and the Hamilton function $\mathcal{H}_{2}$ given in (16) noting that for $O_{1}^{+}, \mathcal{H}_{2}$ is expanded around the origin.

After rescaling the whole Hamiltonian by a constant factor, we introduce the symplectic linear change

$$
Q_{1}=\varepsilon^{1 / 4} \frac{x_{1}}{2 \sqrt{h(3+\delta)}}, \quad Q_{2}=\varepsilon^{1 / 4} \frac{x_{2}}{2 \sqrt{h(3-\delta)}}, \quad P_{1}=2 \varepsilon^{1 / 4} \sqrt{h(3+\delta)} y_{1}, \quad P_{2}=2 \varepsilon^{1 / 4} \sqrt{h(3-\delta)} y_{2},
$$


that transforms the 2-jet of $\mathcal{H}_{2}$ into

$$
H_{2}^{\mathrm{jet}}=8 h^{2}(\delta+1)(\delta+3) y_{1}^{2}+\frac{x_{1}^{2}}{2}+8 h^{2}(\delta-1)(\delta-3) y_{2}^{2}+\frac{x_{2}^{2}}{2},
$$

while the terms of order four are transformed accordingly. We have multiplied the Hamiltonian by the multiplier $\varepsilon^{1 / 2}$.

Next we introduce a canonical transformation by means of the Lie-Deprit's method [7] with the aim of introducing an approximate symmetry to the Hamiltonian system by extending the integral of the oscillator $8 h^{2}(\delta-1)(\delta-3) y_{2}^{2}+\frac{1}{2} x_{2}^{2}$ to the 4 -jet. This is achieved through a generating function which is a homogeneous polynomial of degree four in $x, y$ properly defined for $\delta \approx-1$. We do not write it down as it is a big polynomial. Using the same name for the transformed coordinates we arrive at the following Hamiltonian:

$$
\begin{aligned}
& H_{4}^{\mathrm{jet}}=H_{2}^{\mathrm{jet}}+\varepsilon^{1 / 2}\left[\frac{\delta-2}{16 h^{2}(\delta-1)(\delta-3)} x_{2}^{4}+\frac{2\left(\delta^{2}+\delta-3\right)}{\delta-1} x_{2}^{2} y_{1}^{2}+\frac{128}{3} h^{2}(\delta+1)(\delta+2)(\delta+3) y_{1}^{4}+\right. \\
&\left.2(\delta-2) x_{2}^{2} y_{2}^{2}+32 h^{2}(\delta-3)\left(\delta^{2}+\delta-2\right) y_{1}^{2} y_{2}^{2}+16 h^{2}(\delta-1)(\delta-2)(\delta-3) y_{2}^{4}\right] .
\end{aligned}
$$

Since for the system associated to $H_{4}^{\text {jet }}$ the oscillator related to $x_{2}, y_{2}$ is a constant of motion we set it equal to an action, that is, we identify

$$
J=8 h^{2}(\delta-1)(\delta-3) y_{2}^{2}+\frac{x_{2}^{2}}{2}
$$

where $J \geq 0$ for $\delta \approx-1$ and the 4 -jet is converted into

$$
\begin{aligned}
H_{4}^{\text {jet }}= & J+\varepsilon^{1 / 2} \frac{(\delta-2) J^{2}}{4 h^{2}(\delta-1)(\delta-3)}+\left[8 h^{2}(\delta+1)(\delta+3)+\varepsilon^{1 / 2} \frac{4\left(\delta^{2}+\delta-3\right) J}{\delta-1}\right] y_{1}^{2}+\frac{x_{1}^{2}}{2}+ \\
& \frac{128}{3} \varepsilon^{1 / 2} h^{2}(\delta+1)(\delta+2)(\delta+3) y_{1}^{4} .
\end{aligned}
$$

When $J$ is fixed to a constant, say $j>0$, the Hamiltonian system related to $H_{4}^{\text {jet }}$ is of one degree of freedom and due to the absence of resonances between the pairs $x_{1} / y_{1}, x_{2} / y_{2}$ the reduced space of the one-degree-of-freedom system is a plane around the origin, a feature which prevents us to think of a period-doubling Hamiltonian bifurcation.

In order to get the value of $\delta$ where the bifurcation takes place, we equate the coefficient of $y_{1}^{2}$ to zero in $H_{4}^{\text {jet }}$ and expand $\delta$ in powers of $\varepsilon^{1 / 2}$ around -1 by setting $\delta=-1+\varepsilon^{1 / 2} d_{1}+\varepsilon d_{2}+$ $\varepsilon^{3 / 2} d_{3}+\varepsilon^{2} d_{4}+\cdots$ where the $d_{j}$ have to be determined. After some manipulations we get the formal expansion

$$
\delta=-1-\varepsilon^{1 / 2} \frac{3 j}{8 h^{2}}+\varepsilon \frac{3 j^{2}}{64 h^{2}}-\varepsilon^{3 / 2} \frac{4 j^{3}}{2048 h^{6}}+\varepsilon^{2} \frac{3 j^{4}}{4096 h^{2}}+\cdots .
$$

The product of the coefficients of $x_{1}^{2}$ and $\varepsilon^{1 / 2} y_{1}^{4}$ in $H_{4}^{\text {jet }}$ yields $\frac{64}{3} h^{2}(\delta+1)(\delta+2)(\delta+3)$. When $\delta$ takes the value given in (26), the product reads as

$$
-16 \varepsilon^{1 / 2} j+\varepsilon \frac{11 j^{2}}{h^{2}}-\varepsilon^{3 / 2} \frac{55 j^{3}}{16 h^{4}}+\varepsilon^{2} \frac{85 j^{4}}{128 h^{6}}+\cdots,
$$

which is a negative number provided $j$ and $\varepsilon$ are strictly positive. Thus the bifurcation is of subcritical nature.

At this point it is natural to think about the possibility of reconstructing the periodic Hamiltonian pitchfork bifurcations to the full system associated to Hamiltonian (1) in $\mathbb{R}^{6}$, concluding the existence of quasi-periodic Hamiltonian pitchfork bifurcations, applying the theorems of [18]. However the passage from $\mathcal{B}(h)$ to $\mathbb{R}^{6}$ is not straightforward. On the one hand we have to take 
into account that in the full Hamiltonian, the term $\mathcal{H}_{2}$ from where we have deduced the periodic Hamiltonian pitchfork bifurcation appears as a perturbation of the zeroth-order term $h=L$, thus it is not of the same order. On the other hand, the quasi-periodic Hamiltonian pitchfork bifurcations involve the persistence of invariant 2-tori and it is not so clear that such tori exist. The reason is that the Hamiltonian system is very degenerate and to our knowledge there are no theorems on lower-dimensional tori for multi-scale Hamiltonian systems that we could apply in this setting. Therefore we do not pursue this issue in the present paper.

Observation 5.1. Other Hamiltonian bifurcations on $\mathcal{B}(h)$ that can be considered of pitchfork type are related to the point $O_{1}^{+}$when $\delta=3$, to the points $O_{3}^{+}, O_{4}^{+}$when $\delta=1$, and to the points $O_{5}^{+}$, $\mathrm{O}_{6}^{+}$when $\delta=3$. However in all these situations we have obtained degenerate 4-jets and the addition of higher-order terms does not seem to remove the degeneracy.

Another bifurcation related to the periodic solutions of circular nature, that is, related to the critical points $O_{3}^{+}, O_{4}^{+}$on $\mathcal{B}(h)$ occurs for $\delta \approx-5$. After expanding $\mathcal{H}_{2}$ given in (16) around $\left(Q_{1}, Q_{2}, P_{1}, P_{2}\right)=(0,0,0, \pm \sqrt{h})$, we first observe that the 2 -jet becomes

$$
h(\delta+5)\left(Q_{1}^{2}+P_{1}^{2}\right)+2 h Q_{2}^{2}+4 h(\delta-1) P_{2}^{2} .
$$

Thus we may compute a normal form Hamiltonian so that $2 h Q_{2}^{2}+4 h(\delta-1) P_{2}^{2}$ becomes an integral of the (transformed) $n$-jet with $n \geq 4$. More specifically, after setting $J=2 h Q_{2}^{2}+4 h(\delta-1) P_{2}^{2}$ and performing some transformations similar to the ones used for the pitchfork bifurcation, the resulting Hamiltonian is of the form

$$
F_{1}(J)+F_{2}(J)\left(x_{1}^{2}+y_{1}^{2}\right)+F_{3}(J)\left(x_{1}^{2}+y_{1}^{2}\right)^{2},
$$

where $F_{i}$ are functions on $J$ and the parameters $h, \delta$. This is the typical normal form of a Hamil-

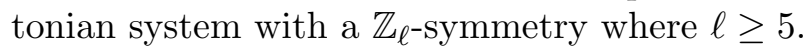

Fixing $J=j \neq 0, F_{2}$ is zero when $\delta$ is near -5 (the concrete value depends on $h$ and $j$ ). However, $J$ does not correspond to an oscillator but to a diffuser so it is a saddle, hence the bifurcation of equilibrium points in the corresponding one-degree-of-freedom system does not reconstruct to a bifurcation of the same type involving periodic solutions in $\mathcal{B}(h)$. Moreover there is no theorem that can be applied to this situation.

Focusing on the case $h<0$ and the critical point $O_{1}^{-}$we prove the occurrence of Hamiltonian Hopf bifurcations of periodic solutions for the Hamiltonian system related to (1).

Theorem 5.1. For the Hamiltonian system associated to (1) with $h<0$, the near rectilinear periodic solution generated by the critical point in $\mathcal{R}_{1}$ experiences periodic Hamiltonian Hopf bifurcations when $\delta \in\{-3,-1\}$. The bifurcation is subcritical when $\delta=-3$ and supercritical when $\delta=-1$.

Proof. The Hamiltonian Hopf bifurcation firstly analysed in [35] for systems with two degrees of freedom, see also $[46,31]$, in our setting involves the interaction of periodic solutions and invariant 2-tori. In a system of three degrees of freedom a Hamiltonian Hopf bifurcation of supercritical type corresponds to the case where a family of normally elliptic invariant 2-tori detaches from the bifurcating periodic solution when the bifurcation parameter passes through its critical value. A subcritical Hamiltonian Hopf bifurcation occurs when there is a family of hyperbolic invariant 2-tori that emanate and return to the bifurcating periodic solution. See more details in $[19,18]$.

We start with the critical point $O_{1}^{-}$when $\delta \approx-1$. We consider the coordinates $Q / P$ defined in (12) related to the Hamiltonian of (17) noting that for $O_{1}^{-}, \mathcal{H}_{2}$ is expanded around the origin. 
Rescaling the whole Hamiltonian by a constant factor that does not vanish for $\delta \approx-1$ and introducing the symplectic linear change

$$
\begin{aligned}
& Q_{1}=\varepsilon^{1 / 4} \frac{x_{1}}{2 \sqrt{-h(3+\delta)}}, \quad Q_{2}=\varepsilon^{1 / 4} \frac{x_{2}}{2 \sqrt{-h(3+\delta)}}, \\
& P_{1}=2 \varepsilon^{1 / 4} \sqrt{-h(3+\delta)} y_{1}, \quad P_{2}=2 \varepsilon^{1 / 4} \sqrt{-h(3+\delta)} y_{2},
\end{aligned}
$$

the 2 -jet of $\mathcal{H}_{2}$ is changed to

$$
H_{2}^{\mathrm{jet}}=8 h^{2}(\delta+1)(\delta+3)\left(y_{1}^{2}+y_{2}^{2}\right)+\frac{x_{1}^{2}}{2}+\frac{x_{2}^{2}}{2} .
$$

The terms of degree four in $x / y$ are also transformed. To make the linear change symplectic we multiply the Hamiltonian by the factor $\varepsilon^{1 / 2}$.

In order to apply the theory of [18] we introduce an $\mathbb{S}^{1}$-symmetry to the non-linear normal form. This step was not needed in $[19,4]$ as the systems tackled in those papers already enjoyed that symmetry. In our problem, if we try to build a normal form through a change of coordinates introduced by a generating function defined as a polynomial of degree four we notice that it is impossible. So, we require an extra transformation. Specifically, following a suggestion by Deprit in [8], we pass to a rotating frame and add a Coriolis-like term in the Hamilton function. The change is accomplished by means of the time-dependent linear transformation given by

$x_{1}=X_{1} \cos t+X_{2} \sin t, \quad x_{2}=X_{2} \cos t-X_{1} \sin t, \quad y_{1}=Y_{1} \cos t+Y_{2} \sin t, \quad y_{2}=Y_{2} \cos t-Y_{1} \sin t$.

By applying this change, the 2 -jet gets transformed into

$$
H_{2}^{\text {jet }}=8 h^{2}(\delta+1)(\delta+3)\left(Y_{1}^{2}+Y_{2}^{2}\right)+\frac{X_{1}^{2}}{2}+\frac{X_{2}^{2}}{2}+X_{2} Y_{1}-X_{1} Y_{2},
$$

while the terms in the 4-jet of degree four in $X, Y$ depend explicitly on the time in a periodic way.

Then we want to obtain a symplectic transformation so that the terms of order 4 in the resulting 4 -jet of the normal form are of the form

$$
H_{4}^{\mathrm{NF}}=b_{0}\left(X_{2} Y_{1}-X_{1} Y_{2}\right)^{2}+b_{1}\left(X_{2} Y_{1}-X_{1} Y_{2}\right)\left(Y_{1}^{2}+Y_{2}^{2}\right)+b_{2}\left(Y_{1}^{2}+Y_{2}^{2}\right)^{2},
$$

where the $b_{i}$ are the constant coefficients to be determined. This transformation is accomplished through a generating function, say $W_{4}$, that is a polynomial in $X$ and $Y$ of degree four whose coefficients are time-dependent and have to be determined as well.

This process involves one step of a Lie-Deprit transformation in the setting of time-dependent Lie transformations. The treatment is more cumbersome than the one of autonomous transformations as we need to solve a homological equation of the form

$$
\left\{H_{2}^{\mathrm{jet}}, W_{4}\right\}-\frac{\partial W_{4}}{\partial t}+H_{4}^{\mathrm{jet} *}=H_{4}^{\mathrm{NF}},
$$

where $H_{4}^{\text {jet* }}=\varepsilon^{-1 / 2}\left(H_{4}^{\text {jet }}-H_{2}^{\text {jet }}\right)$.

The homological equation involves the solution of differential equations for the coefficients of $W_{4}$. However we can somehow bypass this trouble by a technique of matching the coefficients. In particular we impose that the coefficients of each monomial of $W_{4}$ are of the form

$$
d_{i}+c_{2, i} \cos (2 t)+s_{2, i} \sin (2 t)+c_{4, i} \cos (4 t)+s_{4, i} \sin (4 t),
$$

for $i=1, \ldots, 35$ as $W_{4}$ has 35 monomials of degree four in $X, Y$. Plugging the expressions of $W_{4}$ and $H_{4}^{\mathrm{NF}}$ in the homological equation and arranging the resulting terms carefully we finally end up with a linear system of 175 equations whose unknowns are the real coefficients $d_{i}, c_{2, i}, s_{2, i}, c_{4, i}, s_{4, i}$ and $b_{j}$. 
The system has a unique solution that is valid for $\delta$ near -1 . We do not write down the explicit form taken by $W_{4}$ as it is a huge expression, but the final form of the 4-jet in normal form, after returning to the coordinates $x, y$, is $H_{4}^{\text {jet }}=H_{2}^{\text {jet }}+\varepsilon^{1 / 2} H_{4}^{\mathrm{NF}}$ where

$$
\begin{aligned}
H_{4}^{\mathrm{jet}}= & 8 h^{2}(\delta+1)(\delta+3)\left(y_{1}^{2}+y_{2}^{2}\right)+\frac{x_{1}^{2}}{2}+\frac{x_{2}^{2}}{2}+ \\
& \frac{2}{3} \varepsilon^{1 / 2}(\delta+4)\left(x_{2} y_{1}-x_{1} y_{2}\right)^{2}+\frac{8 h^{2}}{3} \varepsilon^{1 / 2}\left(14 \delta^{3}+94 \delta^{2}+191 \delta+123\right)\left(y_{1}^{2}+y_{2}^{2}\right)^{2} .
\end{aligned}
$$

Multiplying the coefficients of $x_{1}^{2}+x_{2}^{2}$ and $\varepsilon^{1 / 2}\left(y_{1}^{2}+y_{2}^{2}\right)^{2}$ in $H_{4}^{\text {jet }}$ we get $\frac{4}{3} h^{2}\left(14 \delta^{3}+94 \delta^{2}+191 \delta+123\right)$ which becomes $16 h^{2}$ when $\delta$ is replaced by -1 . As $h<0$ we conclude that the bifurcation is supercritical.

For $\delta=-3$ the transformations are similar. Instead of the linear change relating the $Q_{i}, P_{i}$ with $x_{i}, y_{i}$, we define

$$
\begin{aligned}
Q_{1} & =2 \varepsilon^{1 / 4} \sqrt{h(\delta+1)} x_{1}, & Q_{2} & =2 \varepsilon^{1 / 4} \sqrt{h(\delta+1)} x_{2}, \\
P_{1} & =\varepsilon^{1 / 4} \frac{y_{1}}{2 \sqrt{h(\delta+1)}}, & P_{2} & =\varepsilon^{1 / 4} \frac{y_{2}}{2 \sqrt{h(\delta+1)}},
\end{aligned}
$$

which is valid for $h<0$ and $\delta$ near -3 .

The next steps are analogous to those described for the case $\delta=-1$. The final form of the 4 -jet in the normal form coordinates is

$$
\begin{aligned}
H_{4}^{\mathrm{jet}}= & -8 h^{2}(\delta+1)(\delta+3)\left(x_{1}^{2}+x_{2}^{2}\right)-\frac{x_{1}^{2}}{2}-\frac{y_{2}^{2}}{2}+ \\
& \frac{2}{3} \varepsilon^{1 / 2}(\delta+4)\left(x_{2} y_{1}-x_{1} y_{2}\right)^{2}+\frac{8 h^{2}}{3} \varepsilon^{1 / 2}\left(14 \delta^{3}+94 \delta^{2}+191 \delta+123\right)\left(x_{1}^{2}+x_{2}^{2}\right)^{2} .
\end{aligned}
$$

On this occasion we multiply the coefficients of $y_{1}^{2}+y_{2}^{2}$ and $\varepsilon^{1 / 2}\left(x_{1}^{2}+x_{2}^{2}\right)^{2}$ in $H_{4}^{\text {jet }}$ and replacing the resulting product for $\delta=-4$ we get $-24 h^{2}<0$. Thus the bifurcation is subcritical.

\section{KAM 3-TORI}

We establish the persistence of invariant three-dimensional tori encasing some linearly stable periodic solutions. The key point is the application of a specific KAM result by Han, Li and Yi [17] which allows to deal with Hamiltonians with high-order proper degeneracy, where the perturbation appears at different orders, with at least three time scales.

In particular we focus on the 3 -tori related to the linearly stable periodic solutions for which $\bar{p}$ is $O_{1}^{+}$or $O_{2}^{+}$, i.e. they are in the set $\mathcal{R}_{51}$. Under the assumptions of Theorem 4.2 we obtain the following result.

Theorem 6.1. When $h>0$, for the Hamiltonian system associated to (1) and the parameter $\delta \in(-\infty,-3) \cup(-1,0) \cup(0,1) \cup(3, \infty)$ and $\beta \neq 0$, there are families of invariant 3 -tori around the near rectilinear periodic solutions in the $O x$ and $O z$ axes corresponding to the set $\mathcal{R}_{51}$. These invariant tori form a majority in the sense that the measure of the complement of their union is of the order $O\left(\varepsilon^{\sigma / 2}\right)$ with $0<\sigma<1 / 5$.

Proof. We consider the calculations for $O_{1}^{+}$and Hamiltonian (16). The calculations for $O_{2}^{+}$and Hamiltonian (18) are practically the same and we omit them.

By scaling coordinates through the linear transformation

$$
\bar{Q}_{1}=\varepsilon^{-1 / 4} Q_{1}, \quad \bar{Q}_{2}=\varepsilon^{-1 / 4} Q_{2}, \quad \bar{P}_{1}=\varepsilon^{-1 / 4} P_{1}, \quad \bar{P}_{2}=\varepsilon^{-1 / 4} P_{2},
$$


which is symplectic with multiplier $\varepsilon^{-1 / 2}$, Hamiltonian (24) takes the form

$$
\overline{\mathcal{H}}=\overline{\mathcal{H}}_{2}(\bar{Q}, \bar{P})+\varepsilon^{1 / 2} \overline{\mathcal{H}}_{4}(\bar{Q}, \bar{P})+O\left(\varepsilon^{3 / 4}\right),
$$

after scaling time by dividing by the multiplier. In particular

$$
\begin{aligned}
\overline{\mathcal{H}}_{2}= & \frac{1}{4} h \beta\left[(3+\delta) \bar{Q}_{1}^{2}+(3-\delta) \bar{Q}_{2}^{2}+(1+\delta) \bar{P}_{1}^{2}+(1-\delta) \bar{P}_{2}^{2}\right], \\
\overline{\mathcal{H}}_{4}=- & \frac{1}{2} \beta \bar{Q}_{1} \bar{Q}_{2} \bar{P}_{1} \bar{P}_{2}+\frac{3}{8} \beta\left(\bar{Q}_{1}^{4}+\bar{Q}_{1}^{2} \bar{Q}_{2}^{2}-\bar{Q}_{2}^{4}\right)+\frac{1}{8} \beta \delta\left(\bar{Q}_{1}^{4}-\bar{Q}_{1}^{2} \bar{Q}_{2}^{2}+\bar{Q}_{2}^{4}\right)+ \\
& \frac{1}{8} \beta(1+\delta) \bar{P}_{1}^{4}-\frac{1}{8} \beta(1-\delta) \bar{P}_{2}^{4}+\frac{1}{8} \beta \bar{P}_{1}^{2}\left[2(2+\delta) \bar{Q}_{1}^{2}+(3-\delta)\left(\bar{Q}_{2}^{2}+\bar{P}_{2}^{2}\right)\right]- \\
& \frac{1}{8} \beta \bar{P}_{2}^{2}\left[(1+\delta) \bar{Q}_{1}^{2}+2(2-\delta) \bar{Q}_{2}^{2}+(3-\delta) \bar{P}_{1}^{2}\right] .
\end{aligned}
$$

For $\delta \in(-\infty,-3) \cup(-1,1) \cup(3, \infty)$, the eigenvalues of matrix $\mathbb{J} D^{2} \overline{\mathcal{H}}_{2}$ are $\pm \alpha_{1} i, \pm \alpha_{2} i$ with $\alpha_{1}=$ $\frac{h \beta}{2} \sqrt{(3+\delta)(1+\delta)}$ and $\alpha_{2}=\frac{h \beta}{2} \sqrt{(3-\delta)(1-\delta)}$. Next, we proceed to normalise the quadratic part $\underline{\mathcal{H}}_{2}$ through the complex symplectic transformation $\left(\bar{Q}_{j}, \bar{P}_{j}\right) \rightarrow\left(q_{j}, p_{j}\right)$ given by

$$
\begin{array}{ll}
\bar{Q}_{1}=\frac{1}{2}\left(\frac{1+\delta}{3+\delta}\right)^{1 / 4}\left(1+\mu_{1} i\right)\left(q_{1}-p_{1}\right), & \bar{P}_{1}=\frac{1}{2}\left(\frac{3+\delta}{1+\delta}\right)^{1 / 4}\left(1-\mu_{1} i\right)\left(q_{1}+p_{1}\right), \\
\bar{Q}_{2}=\frac{1}{2}\left(\frac{1-\delta}{3-\delta}\right)^{1 / 4}\left(1+\mu_{2} i\right)\left(q_{2}-p_{2}\right), & \bar{P}_{2}=\frac{1}{2}\left(\frac{3-\delta}{1-\delta}\right)^{1 / 4}\left(1-\mu_{2} i\right)\left(q_{2}+p_{2}\right),
\end{array}
$$

where

$$
\mu_{1}=\left\{\begin{array}{rl}
1, & \delta \in(-\infty,-3), \\
-1, & \delta \in(-1,1) \cup(3, \infty),
\end{array} \quad \mu_{2}=\left\{\begin{aligned}
-1, & \delta \in(-\infty,-3) \cup(-1,1), \\
1, & \delta \in(3, \infty)
\end{aligned}\right.\right.
$$

Firstly we apply this transformation to Hamiltonian (28) taking into account the different values for $\mu_{1}$ and $\mu_{2}$. Then we apply the Lie-Deprit method [7], and normalise the resulting Hamiltonian, including the terms factorised by $\varepsilon^{1 / 2}$, arriving at

$$
\overline{\mathcal{H}}=i\left(\alpha_{1} q_{1} p_{1}+\alpha_{2} q_{2} p_{2}\right)+\varepsilon^{1 / 2} \overline{\mathcal{H}}_{4}\left(q_{1}, q_{2}, p_{1}, p_{2}\right)+O\left(\varepsilon^{5 / 8}\right),
$$

where

$$
\overline{\mathcal{H}}_{4}=-\frac{\beta}{2}\left[(2+\delta) q_{1}^{2} p_{1}^{2}+(\delta-2) q_{2}^{2} p_{2}^{2}+\frac{\sqrt{\left(1-\delta^{2}\right)\left(9-\delta^{2}\right)}\left(3-\delta-\delta^{2}\right)}{\left(1-\delta^{2}\right)(3+\delta)} q_{1} q_{2} p_{1} p_{2}\right] .
$$

We have to discard that $\delta=0$ because in this case the system is in 1:1 resonance and the normal from transformation does not leave the transformed Hamiltonian as $\overline{\mathcal{H}}_{4}$.

Introducing the action-angle variables $\left(I_{1}, I_{2}, \theta_{1}, \theta_{2}\right)$ given by

$$
\begin{array}{ll}
q_{1}=\sqrt{I_{1}}\left(\cos \theta_{1}-i \sin \theta_{1}\right), & p_{1}=\sqrt{I_{1}}\left(\sin \theta_{1}-i \cos \theta_{1}\right), \\
q_{2}=\sqrt{I_{2}}\left(\cos \theta_{2}-i \sin \theta_{2}\right), & p_{2}=\sqrt{I_{2}}\left(\sin \theta_{2}-i \cos \theta_{2}\right),
\end{array}
$$

also incorporating the terms associated to $L$ dropped in the normalisation procedure and undoing the time scaling, we obtain

$$
\mathcal{H}_{\varepsilon}=h_{0}(L)+\eta^{4} h_{1}(L)+\eta^{5} h_{2}\left(L, I_{1}, I_{2}\right)+\eta^{6} h_{3}\left(L, I_{1}, I_{2}\right)+O\left(\eta^{7}\right),
$$


where

$$
\begin{aligned}
& h_{0}=L \\
& h_{1}=\frac{1}{4} \beta \delta L^{2}, \\
& h_{2}=\frac{1}{2} \beta L\left[\sqrt{(3+\delta)(1+\delta)} I_{1}+\sqrt{(3-\delta)(1-\delta)} I_{2}\right] \\
& h_{3}=\frac{1}{2} \beta\left[(2+\delta) I_{1}^{2}+(\delta-2) I_{2}^{2}+\frac{\sqrt{\left(1-\delta^{2}\right)\left(9-\delta^{2}\right)}\left(3-\delta-\delta^{2}\right)}{\left(1-\delta^{2}\right)(3+\delta)} I_{1} I_{2}\right],
\end{aligned}
$$

with $\eta=\varepsilon^{1 / 2}$. Using the notation given in Theorem A.4 and taking $n=3, a=3, m_{1}=4, m_{2}=$ $5, m_{3}=6, n_{0}=n_{1}=1, n_{2}=n_{3}=3, I^{n_{0}}=I^{n_{1}}=L, I^{n_{2}}=I^{n_{3}}=\left(L, I_{1}, I_{2}\right), \bar{I}^{n_{0}}=\bar{I}^{n_{1}}=L$, $\bar{I}^{n_{2}}=\left(I_{1}, I_{2}\right), \bar{I}^{n_{3}}=I_{2}$, the frequency vector becomes

$$
\Omega(I)=\left(\Omega_{1}, \Omega_{2}, \Omega_{3}, \Omega_{4}, \Omega_{5}\right)=\left(\frac{\partial h_{0}}{\partial L}, \frac{\partial h_{1}}{\partial L}, \frac{\partial h_{2}}{\partial I_{1}}, \frac{\partial h_{2}}{\partial I_{2}}, \frac{\partial h_{3}}{\partial I_{2}}\right) .
$$

The $5 \times 4$ matrix with columns $\Omega(I), \partial \Omega / \partial L, \partial \Omega / \partial I_{1}$ and $\partial \Omega / \partial I_{2}$, is given by

$$
M_{\Omega}=\left[\begin{array}{cccc}
1 & 0 & 0 & 0 \\
\Omega_{2} & \frac{\beta \delta}{2} & 0 & 0 \\
\Omega_{3} & \frac{\beta}{2} \sqrt{(3+\delta)(1+\delta)} & 0 & 0 \\
\Omega_{4} & \frac{\beta}{2} \sqrt{(3-\delta)(1-\delta)} & 0 & 0 \\
\Omega_{5} & 0 & \frac{\beta \sqrt{\left(1-\delta^{2}\right)\left(9-\delta^{2}\right)}\left(3-\delta-\delta^{2}\right)}{2\left(1-\delta^{2}\right)(3+\delta)} & \beta(\delta-2)
\end{array}\right]
$$

it has rank three for all $\delta \in(-\infty,-3) \cup(-1,0) \cup(0,1) \cup(3, \infty)$ and $\beta \neq 0$. In conclusion, Han, Li and Yi's Theorem guarantees the existence of KAM tori of dimension three enclosing the near rectilinear periodic solution in the $O x$ axis. According to Theorem A.4 we have $b=10$ and $s=1$, therefore, the excluded measure for the existence of quasi-periodic invariant tori is of order $O\left(\eta^{\sigma}\right)=O\left(\varepsilon^{\sigma / 2}\right)$ and cannot be improved unless we push the appearance of the angles to higher order by performing more steps in the two transformations to normal form (see Remark 2 in [17]). Thus, the proof of our theorem is completed.

When $h>0$, other KAM 3-tori related to the linearly stable periodic solutions found in Section 4 through Theorem 4.4 can be obtained by applying the same techniques exposed in this section. Hence, we expect invariant 3 -tori encasing the periodic solutions of the sets $\mathcal{R}_{52}, \mathcal{R}_{53}$ and $\mathcal{R}_{6}$ for the values of $\delta$ where these periodic solutions are elliptic.

Observation 6.1. For $h<0$ we have calculated the normal form concerning the periodic solution related to the critical point $O_{1}^{-}$when it is linearly stable. However we could not build actionangle coordinates so that we arrive at an expression of the type (33), due to the presence of a 1:1 resonance between the pairs $Q_{i} / P_{i}, i=1,2$ (see in Table 4 the case of the critical point in $\left.\mathcal{R}_{1}\right)$. According to Theorem 4.3 no other periodic solution can be linearly stable, thus we have not obtained invariant 3 -tori with $h<0$. An exception to this occurs for a particular case of $\delta$. More specifically, if instead of applying the transformation (32) we use the set of Lissajous coordinates introduced in [9], at order $\varepsilon^{1 / 2}$ there is only one term that depends on the Lissajous angle $\ell_{2}$ while the dependence with respect to the other happens at higher orders. This term is factorised by the polynomial $2 \delta^{3}+6 \delta^{2}-\delta-9$ which becomes zero for three real values of $\delta$. The interesting one is $\delta \approx 1.10909 \ldots$ since in this situation the periodic solution is linearly stable. Hence, for that specific value of $\delta$ the three angles of the normalised Hamiltonian appear at an order high enough that one 
can apply an approach similar to that of the Theorem 6.1 concluding the persistence of families of 3-tori with the same estimate as in the previous theorem.

\section{Conclusions}

In an attempt of giving a naive and intuitive cosmological interpretation of our results when $z=Z=0$ we would say the following. The $x$-oscillator accounts for the time evolution of the energy density, whereas the $y$-oscillator refers to the evolution of the scale factor, the size of our universe. The pair $z / Z$ provides an extra degree of freedom to define a more realistic model than those accounting for a $1:-1$ resonance given for instance in $[3,27]$.

When $h>0$ it means that the density in the universe is big compared to its size. This would happen in relatively early states after the Big Bang. There is a high interaction among the particles and this is reflected in the fact that we observe many periodic solutions (up to twenty six families) and many bifurcations (nine). The system is more chaotic and any perturbation affects drastically the evolution of it. Meanwhile, when $h<0$ it means that the part corresponding to the scale factor is bigger than the one associated to the density. So, the universe is "big" and the density is small. Thus, there is less interaction among the particles and we can appreciate that there are not as many periodic solutions (up to thirteen families) and bifurcations (five) as in the previous situation. Furthermore we have not found invariant 3-tori for negative $h$ (with one exception) while there are usually Lagrangian tori encasing the linearly stable periodic solutions when $h$ is positive. In any case, the situations $h<0$ and $h>0$ are not symmetric from the cosmological point of view and neither the bifurcation planes appearing in Figs. 1 and 2 are.

Compared with our previous studies on perturbations of the 1:1:1 resonance, see for instance [38], we outline that the number of bifurcations in the present case is higher. Bifurcations are related with breaking of separatrices, which leads to chaos, as it has been already observed, for instance in [3]. In this respect the analysis performed in Section 5 can be understood as a first step in the study of bifurcations involving periodic solutions and invariant tori in Hamiltonian systems with three and more degrees of freedom. In particular we have been able to establish rigorously the existence of periodic Hamiltonian Hopf bifurcations for a fully resonant model with three degrees of freedom.

All the computations accomplished to obtain the results appearing in this paper, including the calculation of the normal forms, have been performed by using MATHEMATicA. As many of the results are part of the Ph.D. Thesis of the third author [47] we refer to him for details on the codes.

\section{Appendix A. Some useful Results on stability, AVERAging And KAM theory}

Consider the linear Hamiltonian system

$$
\dot{z}=A z=\mathbb{J} \nabla \mathcal{H}(z), \quad \mathcal{H}=\frac{1}{2} z^{T} S z,
$$

where $S$ is a symmetric matrix and $A=\mathbb{J} S$ is a Hamiltonian matrix.

Definition A.1 (Strong stability). System (35) (or matrix A) is strongly stable (or parametrically stable) if it and all sufficiently small linear constant Hamiltonian perturbations of it are stable. If system (35) is stable but it is not strongly stable, we shall say that it is weakly stable.

Let $\pm \alpha_{1} i, \pm \alpha_{2} i, \ldots, \pm \alpha_{s} i$ be the eigenvalues of matrix $A$, and let $V_{j}, j=1, \ldots, s$, be the maximal real linear subspace where $A$ has eigenvalue $\pm \alpha_{j} i$. So $V_{j}$ is an $A$-invariant symplectic subspace, $A$ restricted to $V_{j}$ has eigenvalues $\pm \alpha_{j} i$, and $\mathbb{R}^{2 n}=V_{1} \oplus V_{2} \oplus \ldots \oplus V_{s}$. Let $\mathcal{H}_{j}$ be the restriction of $\mathcal{H}$ to $V_{j}$.

Theorem A.1 (Krein-Gel'fand). System (35) is strongly stable if and only if

- all the eigenvalues of $A$ are purely imaginary, 
- A is nonsingular,

- A is diagonalisable over the complex numbers, and

- the Hamiltonian $\mathcal{H}_{j}$ is positive or negative definite for each $j$.

See the proof in [49] or [31].

Let $(M, \Omega)$ be a symplectic manifold of dimension $2 n, \mathcal{H}_{0}: M \rightarrow \mathbb{R}$ a smooth Hamiltonian which defines a Hamiltonian vector field $Y_{0}=\left(d H_{0}\right)^{\#}$ with symplectic flow $\varphi_{0}^{t}$. Let $I \subset \mathbb{R}$ be an interval such that each $h \in I$ is a regular value of $\mathcal{H}_{0}$ and $\mathcal{N}_{0}(h)=\mathcal{H}_{0}^{-1}(h)$ is a compact connected circle bundle over a base space $\mathcal{B}(h)$ with projection $\pi: \mathcal{N}_{0}(h) \rightarrow \mathcal{B}(h)$. So, this is the setting of regular reduction theory. Assume that all the solutions of $Y_{0}$ in $\mathcal{N}_{0}(h)$ are periodic and have periods smoothly depending only on the value of the Hamiltonian; i.e., the period is a smooth function $T=T(h)$.

Let $\varepsilon$ be a small parameter, $\mathcal{H}_{1}: M \rightarrow \mathbb{R}$ be smooth, $\mathcal{H}_{\varepsilon}=\mathcal{H}_{0}+\varepsilon \mathcal{H}_{1}, Y_{\varepsilon}=Y_{0}+\varepsilon Y_{1}=d \mathcal{H}_{\varepsilon}^{\#}$, $\mathcal{N}_{\varepsilon}(h)=\mathcal{H}_{\varepsilon}^{-1}(h), \pi: \mathcal{N}_{\varepsilon}(h) \rightarrow \mathcal{B}(h)$ the projection, and $\phi_{\varepsilon}^{t}$ be the flow defined by $Y_{\varepsilon}$.

Let the average of $\mathcal{H}_{1}$ be

$$
\overline{\mathcal{H}}=\frac{1}{T} \int_{0}^{T} \mathcal{H}_{1}\left(\phi_{0}^{t}\right) d t .
$$

The next result provides sufficient conditions for characterising the existence of periodic solutions of the Hamiltonian system associated to $\mathcal{H}_{\varepsilon}$. For more information on this subject the reader is addressed to [39, 40], [50] and [32]. The comprehensive work [44] is recommended as a reference for averaging techniques.

Theorem A.2. If $\overline{\mathcal{H}}$ has a non-degenerate critical point at $\pi(p)=\bar{p} \in \mathcal{B}(h)$ with $p \in \mathcal{N}_{0}(h)$, then there are smooth functions $p(\varepsilon)$ and $T(\varepsilon)$ for $\varepsilon$ small with $p(0)=p, T(0)=T, p(\varepsilon) \in \mathcal{N}_{\varepsilon}$, and the solution of $Y_{\varepsilon}$ through $p(\varepsilon)$ is $T(\varepsilon)$-periodic. In addition to that, if the characteristic exponents of the critical point $\bar{p}$ (that is, the eigenvalues of the matrix $A=\mathbb{J} D^{2} \overline{\mathcal{H}}(\bar{p})$ ) are $\lambda_{1}, \lambda_{2}, \ldots, \lambda_{2 n-2}$, then the characteristic multipliers of the periodic solution through $p(\varepsilon)$ are

$$
1,1,1+\varepsilon \lambda_{1} T+O\left(\varepsilon^{2}\right), 1+\varepsilon \lambda_{2} T+O\left(\varepsilon^{2}\right), \ldots, 1+\varepsilon \lambda_{2 n-2} T+O\left(\varepsilon^{2}\right) .
$$

Theorem A.3. Let $p$ and $\bar{p}$ be as in the previous theorem. If one or more of the characteristic exponents $\lambda_{j}$ is real or has nonzero real part, then the periodic solution through $p(\varepsilon)$ is unstable. If the matrix $A$ is strongly stable, then the periodic solution through $p(\varepsilon)$ is elliptic, i.e., linearly stable.

The proofs of Theorems A.2 and A.3 appear in [50]. Theorem A.2 is a modern formulation of Reeb's Theorem [39, 40].

Consider a Hamiltonian system of the form

$$
\mathcal{H}_{\varepsilon}(I, \varphi, \varepsilon)=h_{0}\left(I^{n_{0}}\right)+\varepsilon^{m_{1}} h_{1}\left(I^{n_{1}}\right)+\ldots+\varepsilon^{m_{a}} h_{a}\left(I^{n_{a}}\right)+\varepsilon^{m_{a}+1} \mathcal{P}(I, \varphi, \varepsilon),
$$

where $(I, \varphi) \in \mathbb{R}^{n} \times \mathbb{T}^{n}$ are action-angle variables with the standard symplectic structure $d I \wedge d \varphi$, and $\varepsilon>0$ is a sufficiently small parameter. Hamiltonian $\mathcal{H}_{\varepsilon}$ is real analytic, and the parameters $a, m, n_{i}$ $(i=0,1, \ldots, a)$ and $m_{j}(j=1,2, \ldots, a)$ are positive integers satisfying $n_{0} \leq n_{1} \leq \ldots \leq n_{a}=n$, $m_{1} \leq m_{2} \leq \ldots \leq m_{a}=m, I^{n_{i}}=\left(I_{1}, \ldots, I_{n_{i}}\right)$, for $i=1,2, \ldots, a$, and $\mathcal{P}$ depends on $\varepsilon$ smoothly.

Hamiltonian $\mathcal{H}_{\varepsilon}(I, \varphi, \varepsilon)$ is taken in a bounded closed region $Z \times \mathbb{T}^{n} \subset \mathbb{R}^{n} \times \mathbb{T}^{n}$. For each $\varepsilon$ the integrable part of $\mathcal{H}_{\varepsilon}$,

$$
X_{\varepsilon}(I)=h_{0}\left(I^{n_{0}}\right)+\varepsilon^{m_{1}} h_{1}\left(I^{n_{1}}\right)+\ldots+\varepsilon^{m_{a}} h_{a}\left(I^{n_{a}}\right),
$$


admits a family of invariant $n$-tori $T_{\zeta}^{\varepsilon}=\{\zeta\} \times \mathbb{T}^{n}$, with linear flows $\left\{x_{0}+\omega^{\varepsilon}(\zeta) t\right\}$, where, for each $\zeta \in Z, \omega^{\varepsilon}(\zeta)=\nabla X_{\varepsilon}(\zeta)$ is the frequency vector of the $n$-torus $T_{\zeta}^{\varepsilon}$ and $\nabla$ is the gradient operator. When $\omega^{\varepsilon}(\zeta)$ is nonresonant, the $n$-torus $T_{\zeta}^{\varepsilon}$ becomes quasi-periodic with slow and fast frequencies of different scales. We refer to the integrable part $X_{\varepsilon}$ and its associated tori $\left\{T_{\zeta}^{\varepsilon}\right\}$ as the intermediate Hamiltonian and intermediate tori, respectively.

Let $\bar{I}^{n_{i}}=\left(I_{n_{i-1}+1}, \ldots, I_{n_{i}}\right), i=0,1, \ldots, a$ (where $n_{-1}=0$, hence $\left.\bar{I}^{n_{0}}=I^{n_{0}}\right)$, and define

$$
\Omega=\left(\nabla_{\bar{I}^{n} 0} h_{0}\left(I^{n_{0}}\right), \ldots, \nabla_{\bar{I}^{n_{a}}} h_{n_{a}}\left(I^{n_{a}}\right)\right),
$$

such that, for each $i=0,1, \ldots, a, \nabla_{\bar{I}^{n_{i}}}$ denotes the gradient with respect to $\bar{I}^{n_{i}}$.

We assume the following high-order degeneracy-removing condition of Bruno-Rüssman type (so named by Han, Li, and Yi), giving credit to Bruno and Rüssman, who provided weak conditions on the frequencies guaranteeing the persistence of invariant tori, the so-called $(A)$ condition: there is a positive integer $s$ such that

$$
\operatorname{Rank}\left\{\partial^{\alpha} \Omega(I): 0 \leq|\alpha| \leq s\right\}=n, \quad \forall I \in Z .
$$

For the usual case of a nearly integrable Hamiltonian system of the type

$$
\mathcal{H}_{\varepsilon}(I, \varphi, \varepsilon)=X(I)+\varepsilon \mathcal{P}(I, \varphi, \varepsilon), \quad(I, \varphi) \in Z \times \mathbb{T}^{n} \subset \mathbb{R}^{n} \times \mathbb{T}^{n} .
$$

Condition $(A)$ given above generalises the classical Kolmogorov non-degeneracy condition that $\partial \omega(I)$ be nonsingular over $Z$, where $\omega(I)=\nabla X(I)$; Bruno's non-degeneracy condition that $\operatorname{Rank}\{\omega(I), \partial \omega(I)\}=n, \forall I \in Z$; and the weakest non-degeneracy condition guaranteeing such persistence provided by Rüssman, that $\omega(Z)$ should not lie in any $(n-1)$-dimensional subspace. Rüssman condition is equivalent to condition $(A)$ for systems like (37). However, Bruno or Rüssman conditions do not apply to Hamiltonian (36), as it is too degenerate.

The following theorem gives the right setting in which one can ensure the persistence of KAM tori for a Hamiltonian like (36).

Theorem A.4 (Han, Li and Yi). Assume condition $(A)$ holds, and let $\delta$ with $0<\delta<1 / 5$ be given. Then there exists an $\varepsilon_{0}>0$ and a family of Cantor sets $Z_{\varepsilon} \subset Z, 0<\varepsilon<\varepsilon_{0}$, with $\left|Z \backslash Z_{\varepsilon}\right|=$ $O\left(\varepsilon^{\delta / s}\right)$, such that each $\zeta \in Z_{\varepsilon}$ corresponds to a real analytic, invariant, quasi-periodic $n$-torus $\bar{T}_{\zeta}^{\varepsilon}$ of Hamiltonian (36), which is slightly deformed from the intermediate $n$-torus $T_{\zeta}^{\varepsilon}$. Moreover, the family $\left\{\bar{T}_{\zeta}^{\varepsilon}: \zeta \in Z_{\varepsilon}, 0<\varepsilon<\varepsilon_{0}\right\}$ varies Whitney smoothly.

See the proof in [17].

\section{Appendix B. Critical points: Tables}

This appendix contains the tables accounting for the critical points expressed in the invariants and also in different charts. 


\begin{tabular}{|c|c|c|}
\hline \multicolumn{2}{|c|}{ Critical Points } & $D_{j}$ \\
\hline \multicolumn{2}{|c|}{ 1) $(0,-2 h, 0,0,0,0,0,0,0)$} & $1,2,3,4$ \\
\hline 2) & $\left.\frac{2 h(\delta+3)}{3(\delta+5)},-\frac{2 h(\delta+9)}{3(\delta+5)}, \frac{2 h(\delta+3)}{3(\delta+5)}, \frac{2 h \sqrt{-(\delta+3)(\delta+9)}}{3(\delta+5)},-\frac{2 h(\delta+3)}{3(\delta+5)},-\frac{2 h \sqrt{-(\delta+3)(\delta+9)}}{3(\delta+5)}, 0,0,0\right)$ & 2,4 \\
\hline 3) & $\left.\frac{2 h(\delta+3)}{3(\delta+5)},-\frac{2 h(\delta+9)}{3(\delta+5)}, \frac{2 h(\delta+3)}{3(\delta+5)}, \frac{2 h \sqrt{-(\delta+3)(\delta+9)}}{3(\delta+5)}, \frac{2 h(\delta+3)}{3(\delta+5)}, \frac{2 h \sqrt{-(\delta+3)(\delta+9)}}{3(\delta+5)}, 0,0,0\right)$ & 2,4 \\
\hline 4) & $\left.\frac{2 h(\delta+3)}{3(\delta+5)},-\frac{2 h(\delta+9)}{3(\delta+5)}, \frac{2 h(\delta+3)}{3(\delta+5)},-\frac{2 h \sqrt{-(\delta+3)(\delta+9)}}{3(\delta+5)},-\frac{2 h(\delta+3)}{3(\delta+5)}, \frac{2 h \sqrt{-(\delta+3)(\delta+9)}}{3(\delta+5)}, 0,0,0\right)$ & 2,4 \\
\hline 5) ( & $\left.\frac{2 h(\delta+3)}{3(\delta+5)},-\frac{2 h(\delta+9)}{3(\delta+5)}, \frac{2 h(\delta+3)}{3(\delta+5)},-\frac{2 h \sqrt{-(\delta+3)(\delta+9)}}{3(\delta+5)}, \frac{2 h(\delta+3)}{3(\delta+5)},-\frac{2 h \sqrt{-(\delta+3)(\delta+9)}}{3(\delta+5)}, 0,0,0\right)$ & 2,4 \\
\hline 6) & $\left.\frac{2 h(\delta+1)}{3 \delta+7},-\frac{2 h(\delta+5)}{3 \delta+7}, \frac{2 h(\delta+1)}{3 \delta+7}, 0,-\frac{2 h(\delta+1)}{3 \delta+7}, 0, \frac{2 h \sqrt{-(\delta+1)(\delta+5)}}{3 \delta+7}, 0,-\frac{2 h \sqrt{-(\delta+1)(\delta+5)}}{3 \delta+7}\right)$ & 3 \\
\hline 7) & $\left.\frac{2 h(\delta+1)}{3 \delta+7},-\frac{2 h(\delta+5)}{3 \delta+7}, \frac{2 h(\delta+1)}{3 \delta+7}, 0,-\frac{2 h(\delta+1)}{3 \delta+7}, 0,-\frac{2 h \sqrt{-(\delta+1)(\delta+5)}}{3 \delta+7}, 0, \frac{2 h \sqrt{-(\delta+1)(\delta+5)}}{3 \delta+7}\right)$ & 3 \\
\hline 8) & $\left.\frac{2 h(\delta+1)}{3 \delta+7},-\frac{2 h(\delta+5)}{3 \delta+7}, \frac{2 h(\delta+1)}{3 \delta+7}, 0, \frac{2 h(\delta+1)}{3 \delta+7}, 0, \frac{2 h \sqrt{-(\delta+1)(\delta+5)}}{3 \delta+7}, 0, \frac{2 h \sqrt{-(\delta+1)(\delta+5)}}{3 \delta+7}\right)$ & 3 \\
\hline 9) & $\left.\frac{2 h(\delta+1)}{3 \delta+7},-\frac{2 h(\delta+5)}{3 \delta+7}, \frac{2 h(\delta+1)}{3 \delta+7}, 0, \frac{2 h(\delta+1)}{3 \delta+7}, 0,-\frac{2 h \sqrt{-(\delta+1)(\delta+5)}}{3 \delta+7}, 0,-\frac{2 h \sqrt{-(\delta+1)(\delta+5)}}{3 \delta+7}\right)$ & 3 \\
\hline 10) & $\left.\frac{2 h(\delta+1)^{2}}{3 \delta^{2}+6 \delta-13},-\frac{2 h(\delta-1)(\delta+5)}{3 \delta^{2}+6 \delta-13}, \frac{2 h\left(\delta^{2}-9\right)}{3 \delta^{2}+6 \delta-13},-\frac{2 h(\delta+1) \sqrt{-(\delta-1)(\delta+5)}}{3 \delta^{2}+6 \delta-13}, 0,0,0, \frac{2 h(\delta+1) \sqrt{\delta^{2}-9}}{3 \delta^{2}+6 \delta-13},-\frac{2 h \sqrt{-(\delta-3)(\delta-1)(\delta+3)(\delta+5)}}{3 \delta^{2}+6 \delta-13}\right)$ & 4 \\
\hline 11) & $\left(\frac{2 h(\delta+1)^{2}}{3 \delta^{2}+6 \delta-13},-\frac{2 h(\delta-1)(\delta+5)}{3 \delta^{2}+6 \delta-13}, \frac{2 h\left(\delta^{2}-9\right)}{3 \delta^{2}+6 \delta-13},-\frac{2 h(\delta+1) \sqrt{-(\delta-1)(\delta+5)}}{3 \delta^{2}+6 \delta-13}, 0,0,0,-\frac{2 h(\delta+1) \sqrt{\delta^{2}-9}}{3 \delta^{2}+6 \delta-13}, \frac{2 h \sqrt{-(\delta-3)(\delta-1)(\delta+3)(\delta+5)}}{3 \delta^{2}+6 \delta-13}\right)$ & 4 \\
\hline 12) ( & $\left(\frac{2 h(\delta+1)^{2}}{3 \delta^{2}+6 \delta-13},-\frac{2 h(\delta-1)(\delta+5)}{3 \delta^{2}+6 \delta-13}, \frac{2 h\left(\delta^{2}-9\right)}{3 \delta^{2}+6 \delta-13}, \frac{2 h(\delta+1) \sqrt{-(\delta-1)(\delta+5)}}{3 \delta^{2}+6 \delta-13}, 0,0,0, \frac{2 h(\delta+1) \sqrt{\delta^{2}-9}}{3 \delta^{2}+6 \delta-13}, \frac{2 h \sqrt{-(\delta-3)(\delta-1)(\delta+3)(\delta+5)}}{3 \delta^{2}+6 \delta-13}\right)$ & 4 \\
\hline 13) ( & $\left.\frac{2 h(\delta+1)^{2}}{3 \delta^{2}+6 \delta-13},-\frac{2 h(\delta-1)(\delta+5)}{3 \delta^{2}+6 \delta-13}, \frac{2 h\left(\delta^{2}-9\right)}{3 \delta^{2}+6 \delta-13}, \frac{2 h(\delta+1) \sqrt{-(\delta-1)(\delta+5)}}{3 \delta^{2}+6 \delta-13}, 0,0,0,-\frac{2 h(\delta+1) \sqrt{\delta^{2}-9}}{3 \delta^{2}+6 \delta-13},-\frac{2 h \sqrt{-(\delta-3)(\delta-1)(\delta+3)(\delta+5)}}{3 \delta^{2}+6 \delta-13}\right)$ & 4 \\
\hline 14) & $\left.\frac{2 h\left(\delta^{2}-9\right)}{3 \delta^{2}+6 \delta-13},-\frac{2 h(\delta-1)(\delta+5)}{3 \delta^{2}+6 \delta-13}, \frac{2 h(\delta+1)^{2}}{3 \delta^{2}+6 \delta-13}, 0,0, \frac{2 h(\delta+1) \sqrt{-(\delta-1)(\delta+5)}}{3 \delta^{2}+6 \delta-13},-\frac{2 h \sqrt{-(\delta-3)(\delta-1)(\delta+3)(\delta+5)}}{3 \delta^{2}+6 \delta-13}, \frac{2 h(\delta+1) \sqrt{\delta^{2}-9}}{3 \delta^{2}+6 \delta-13}, 0\right)$ & 4 \\
\hline 15) & $\left(\frac{2 h\left(\delta^{2}-9\right)}{3 \delta^{2}+6 \delta-13},-\frac{2 h(\delta-1)(\delta+5)}{3 \delta^{2}+6 \delta-13}, \frac{2 h(\delta+1)^{2}}{3 \delta^{2}+6 \delta-13}, 0,0, \frac{2 h(\delta+1) \sqrt{-(\delta-1)(\delta+5)}}{3 \delta^{2}+6 \delta-13}, \frac{2 h \sqrt{-(\delta-3)(\delta-1)(\delta+3)(\delta+5)}}{3 \delta^{2}+6 \delta-13},-\frac{2 h(\delta+1) \sqrt{\delta^{2}-9}}{3 \delta^{2}+6 \delta-13}, 0\right)$ & 4 \\
\hline 16) & $\left(\frac{2 h\left(\delta^{2}-9\right)}{3 \delta^{2}+6 \delta-13},-\frac{2 h(\delta-1)(\delta+5)}{3 \delta^{2}+6 \delta-13}, \frac{2 h(\delta+1)^{2}}{3 \delta^{2}+6 \delta-13}, 0,0,-\frac{2 h(\delta+1) \sqrt{-(\delta-1)(\delta+5)}}{3 \delta^{2}+6 \delta-13},-\frac{2 h \sqrt{-(\delta-3)(\delta-1)(\delta+3)(\delta+5)}}{3 \delta^{2}+6 \delta-13},-\frac{2 h(\delta+1) \sqrt{\delta^{2}-9}}{3 \delta^{2}+6 \delta-13}, 0\right)$ & 4 \\
\hline 17) & $\left(\frac{2 h\left(\delta^{2}-9\right)}{3 \delta^{2}+6 \delta-13},-\frac{2 h(\delta-1)(\delta+5)}{3 \delta^{2}+6 \delta-13}, \frac{2 h(\delta+1)^{2}}{3 \delta^{2}+6 \delta-13}, 0,0,-\frac{2 h(\delta+1) \sqrt{-(\delta-1)(\delta+5)}}{3 \delta^{2}+6 \delta-13}, \frac{2 h \sqrt{-(\delta-3)(\delta-1)(\delta+3)(\delta+5)}}{3 \delta^{2}+6 \delta-13}, \frac{2 h(\delta+1) \sqrt{\delta^{2}-9}}{3 \delta^{2}+6 \delta-13}, 0\right)$ & 4 \\
\hline
\end{tabular}

TABLE 6. Critical points of $\overline{\mathcal{H}}$ in $\mathcal{B}(h)$ for $h<0$. 


\begin{tabular}{|c|c|c|}
\hline \multicolumn{2}{|c|}{ Critical Points } & $D_{j}$ \\
\hline \multicolumn{2}{|c|}{ 1) $(2 h, 0,0,0,0,0,0,0,0)$} & $5,6,7,8$ \\
\hline \multicolumn{2}{|c|}{ 2) $(0,0,2 h, 0,0,0,0,0,0)$} & $5,6,7,8$ \\
\hline \multicolumn{2}{|c|}{ 3) $(h, 0, h, 0,0,0,0,-h, 0)$} & $5,6,7,8$ \\
\hline \multicolumn{2}{|c|}{ 4) $(h, 0, h, 0,0,0,0, h, 0)$} & $5,6,7,8$ \\
\hline \multicolumn{2}{|c|}{ 5) $(h, 0, h, 0,-h, 0,0,0,0)$} & $5,6,7,8$ \\
\hline \multicolumn{2}{|c|}{ 6) $(h, 0, h, 0, h, 0,0,0,0)$} & $5,6,7,8$ \\
\hline 7) ( & $\left.\frac{2 h(\delta+3)}{3(\delta+5)},-\frac{2 h(\delta+9)}{3(\delta+5)}, \frac{2 h(\delta+3)}{3(\delta+5)},-\frac{2 h \sqrt{-(\delta+3)(\delta+9)}}{3(\delta+5)},-\frac{2 h(\delta+3)}{3(\delta+5)}, \frac{2 h \sqrt{-(\delta+3)(\delta+9)}}{3(\delta+5)}, 0,0,0\right)$ & 6 \\
\hline 8) & $\left.\frac{2 h(\delta+3)}{3(\delta+5)},-\frac{2 h(\delta+9)}{3(\delta+5)}, \frac{2 h(\delta+3)}{3(\delta+5)},-\frac{2 h \sqrt{-(\delta+3)(\delta+9)}}{3(\delta+5)}, \frac{2 h(\delta+3)}{3(\delta+5)},-\frac{2 h \sqrt{-(\delta+3)(\delta+9)}}{3(\delta+5)}, 0,0,0\right)$ & 6 \\
\hline & $\left.\frac{2 h(\delta+3)}{3(\delta+5)},-\frac{2 h(\delta+9)}{3(\delta+5)}, \frac{2 h(\delta+3)}{3(\delta+5)}, \frac{2 h \sqrt{-(\delta+3)(\delta+9)}}{3(\delta+5)},-\frac{2 h(\delta+3)}{3(\delta+5)},-\frac{2 h \sqrt{-(\delta+3)(\delta+9)}}{3(\delta+5)}, 0,0,0\right)$ & 6 \\
\hline & $\left(\frac{2 h(\delta+3)}{3(\delta+5)},-\frac{2 h(\delta+9)}{3(\delta+5)}, \frac{2 h(\delta+3)}{3(\delta+5)}, \frac{2 h \sqrt{-(\delta+3)(\delta+9)}}{3(\delta+5)}, \frac{2 h(\delta+3)}{3(\delta+5)}, \frac{2 h \sqrt{-(\delta+3)(\delta+9)}}{3(\delta+5)}, 0,0,0\right)$ & 6 \\
\hline & $\left.\frac{2 h(\delta+1)}{3 \delta+7},-\frac{2 h(\delta+5)}{3 \delta+7}, \frac{2 h(\delta+1)}{3 \delta+7}, 0,-\frac{2 h(\delta+1)}{3 \delta+7}, 0,-\frac{2 h \sqrt{-(\delta+1)(\delta+5)}}{3 \delta+7}, 0, \frac{2 h \sqrt{-(\delta+1)(\delta+5)}}{3 \delta+7}\right)$ & 7,8 \\
\hline & $\left(\frac{2 h(\delta+1)}{3 \delta+7},-\frac{2 h(\delta+5)}{3 \delta+7}, \frac{2 h(\delta+1)}{3 \delta+7}, 0,-\frac{2 h(\delta+1)}{3 \delta+7}, 0, \frac{2 h \sqrt{-(\delta+1)(\delta+5)}}{3 \delta+7}, 0,-\frac{2 h \sqrt{-(\delta+1)(\delta+5)}}{3 \delta+7}\right)$ & 7,8 \\
\hline & $\left.\frac{2 h(\delta+1)}{3 \delta+7},-\frac{2 h(\delta+5)}{3 \delta+7}, \frac{2 h(\delta+1)}{3 \delta+7}, 0, \frac{2 h(\delta+1)}{3 \delta+7}, 0,-\frac{2 h \sqrt{-(\delta+1)(\delta+5)}}{3 \delta+7}, 0,-\frac{2 h \sqrt{-(\delta+1)(\delta+5)}}{3 \delta+7}\right)$ & 7,8 \\
\hline 14) & $\left.\frac{2 h(\delta+1)}{3 \delta+7},-\frac{2 h(\delta+5)}{3 \delta+7}, \frac{2 h(\delta+1)}{3 \delta+7}, 0, \frac{2 h(\delta+1)}{3 \delta+7}, 0, \frac{2 h \sqrt{-(\delta+1)(\delta+5)}}{3 \delta+7}, 0, \frac{2 h \sqrt{-(\delta+1)(\delta+5)}}{3 \delta+7}\right)$ & 7,8 \\
\hline 15) & $\left(\frac{2 h(\delta+1)^{2}}{3 \delta^{2}+6 \delta-13},-\frac{2 h(\delta-1)(\delta+5)}{3 \delta^{2}+6 \delta-13}, \frac{2 h\left(\delta^{2}-9\right)}{3 \delta^{2}+6 \delta-13}, \frac{2 h(\delta+1) \sqrt{-(\delta-1)(\delta+5)}}{3 \delta^{2}+6 \delta-13}, 0,0,0,-\frac{2 h(\delta+1) \sqrt{\delta^{2}-9}}{3 \delta^{2}+6 \delta-13},-\frac{2 h \sqrt{-(\delta-3)(\delta-1)(\delta+3)(\delta+5)}}{3 \delta^{2}+6 \delta-13}\right)$ & 8 \\
\hline 16) & $\left(\frac{2 h(\delta+1)^{2}}{3 \delta^{2}+6 \delta-13},-\frac{2 h(\delta-1)(\delta+5)}{3 \delta^{2}+6 \delta-13}, \frac{2 h\left(\delta^{2}-9\right)}{3 \delta^{2}+6 \delta-13}, \frac{2 h(\delta+1) \sqrt{-(\delta-1)(\delta+5)}}{3 \delta^{2}+6 \delta-13}, 0,0,0, \frac{2 h(\delta+1) \sqrt{\delta^{2}-9}}{3 \delta^{2}+6 \delta-13}, \frac{2 h \sqrt{-(\delta-3)(\delta-1)(\delta+3)(\delta+5)}}{3 \delta^{2}+6 \delta-13}\right)$ & 8 \\
\hline 17) & $\left(\frac{2 h(\delta+1)^{2}}{3 \delta^{2}+6 \delta-13},-\frac{2 h(\delta-1)(\delta+5)}{3 \delta^{2}+6 \delta-13}, \frac{2 h\left(\delta^{2}-9\right)}{3 \delta^{2}+6 \delta-13},-\frac{2 h(\delta+1) \sqrt{-(\delta-1)(\delta+5)}}{3 \delta^{2}+6 \delta-13}, 0,0,0,-\frac{2 h(\delta+1) \sqrt{\delta^{2}-9}}{3 \delta^{2}+6 \delta-13}, \frac{2 h \sqrt{-(\delta-3)(\delta-1)(\delta+3)(\delta+5)}}{3 \delta^{2}+6 \delta-13}\right)$ & 8 \\
\hline 18) & $\left(\frac{2 h(\delta+1)^{2}}{3 \delta^{2}+6 \delta-13},-\frac{2 h(\delta-1)(\delta+5)}{3 \delta^{2}+6 \delta-13}, \frac{2 h\left(\delta^{2}-9\right)}{3 \delta^{2}+6 \delta-13},-\frac{2 h(\delta+1) \sqrt{-(\delta-1)(\delta+5)}}{3 \delta^{2}+6 \delta-13}, 0,0,0, \frac{2 h(\delta+1) \sqrt{\delta^{2}-9}}{3 \delta^{2}+6 \delta-13},-\frac{2 h \sqrt{-(\delta-3)(\delta-1)(\delta+3)(\delta+5)}}{3 \delta^{2}+6 \delta-13}\right)$ & 8 \\
\hline 19) & $\left(\frac{2 h\left(\delta^{2}-9\right)}{3 \delta^{2}+6 \delta-13},-\frac{2 h(\delta-1)(\delta+5)}{3 \delta^{2}+6 \delta-13}, \frac{2 h(\delta+1)^{2}}{3 \delta^{2}+6 \delta-13}, 0,0, \frac{2 h(\delta+1) \sqrt{-(\delta-1)(\delta+5)}}{3 \delta^{2}+6 \delta-13}, \frac{2 h \sqrt{-(\delta-3)(\delta-1)(\delta+3)(\delta+5)}}{3 \delta^{2}+6 \delta-13},-\frac{2 h(\delta+1) \sqrt{\delta^{2}-9}}{3 \delta^{2}+6 \delta-13}, 0\right)$ & 8 \\
\hline 20) & $\left(\frac{2 h\left(\delta^{2}-9\right)}{3 \delta^{2}+6 \delta-13},-\frac{2 h(\delta-1)(\delta+5)}{3 \delta^{2}+6 \delta-13}, \frac{2 h(\delta+1)^{2}}{3 \delta^{2}+6 \delta-13}, 0,0, \frac{2 h(\delta+1) \sqrt{-(\delta-1)(\delta+5)}}{3 \delta^{2}+6 \delta-13},-\frac{2 h \sqrt{-(\delta-3)(\delta-1)(\delta+3)(\delta+5)}}{3 \delta^{2}+6 \delta-13}, \frac{2 h(\delta+1) \sqrt{\delta^{2}-9}}{3 \delta^{2}+6 \delta-13}, 0\right)$ & 8 \\
\hline 21) & $\left(\frac{2 h\left(\delta^{2}-9\right)}{3 \delta^{2}+6 \delta-13},-\frac{2 h(\delta-1)(\delta+5)}{3 \delta^{2}+6 \delta-13}, \frac{2 h(\delta+1)^{2}}{3 \delta^{2}+6 \delta-13}, 0,0, \frac{2 h(\delta+1) \sqrt{-(\delta-1)(\delta+5)}}{3 \delta^{2}+6 \delta-13}, \frac{2 h \sqrt{-(\delta-3)(\delta-1)(\delta+3)(\delta+5)}}{3 \delta^{2}+6 \delta-13}, \frac{2 h(\delta+1) \sqrt{\delta^{2}-9}}{3 \delta^{2}+6 \delta-13}, 0\right)$ & 8 \\
\hline 22) & $\left(\frac{2 h\left(\delta^{2}-9\right)}{3 \delta^{2}+6 \delta-13},-\frac{2 h(\delta-1)(\delta+5)}{3 \delta^{2}+6 \delta-13}, \frac{2 h(\delta+1)^{2}}{3 \delta^{2}+6 \delta-13}, 0,0,-\frac{2 h(\delta+1) \sqrt{-(\delta-1)(\delta+5)}}{3 \delta^{2}+6 \delta-13},-\frac{2 h \sqrt{-(\delta-3)(\delta-1)(\delta+3)(\delta+5)}}{3 \delta^{2}+6 \delta-13},-\frac{2 h(\delta+1) \sqrt{\delta^{2}-9}}{3 \delta^{2}+6 \delta-13}, 0\right)$ & 8 \\
\hline & $\left(\frac{2 h(\delta+1)}{3 \delta+7},-\frac{2 h(\delta+5)}{3 \delta+7}, \frac{2 h(\delta+1)}{3 \delta+7},-h \sqrt{-\frac{\delta+5}{3 \delta+7}},-\frac{h(\delta+5)}{3 \delta+7}, h \sqrt{-\frac{\delta+5}{3 \delta+7}},-\frac{h \sqrt{-(\delta-3)(\delta+5)}}{3 \delta+7},-h \sqrt{\frac{\delta-3}{3 \delta+7}},-\frac{h \sqrt{-(\delta-3)(\delta+5)}}{3 \delta+7}\right)$ & 7,8 \\
\hline 24) & $\left.\frac{2 h(\delta+1)}{3 \delta+7},-\frac{2 h(\delta+5)}{3 \delta+7}, \frac{2 h(\delta+1)}{3 \delta+7},-h \sqrt{-\frac{\delta+5}{3 \delta+7}},-\frac{h(\delta+5)}{3 \delta+7}, h \sqrt{-\frac{\delta+5}{3 \delta+7}}, \frac{h \sqrt{-(\delta-3)(\delta+5)}}{3 \delta+7}, h \sqrt{\frac{\delta-3}{3 \delta+7}}, \frac{h \sqrt{-(\delta-3)(\delta+5)}}{3 \delta+7}\right)$ & 7,8 \\
\hline 25) & $\left.\frac{2 h(\delta+1)}{3 \delta+7},-\frac{2 h(\delta+5)}{3 \delta+7}, \frac{2 h(\delta+1)}{3 \delta+7},-h \sqrt{-\frac{\delta+5}{3 \delta+7}}, \frac{h(\delta+5)}{3 \delta+7},-h \sqrt{-\frac{\delta+5}{3 \delta+7}},-\frac{h \sqrt{-(\delta-3)(\delta+5)}}{3 \delta+7}, h \sqrt{\frac{\delta-3}{3 \delta+7}}, \frac{h \sqrt{-(\delta-3)(\delta+5)}}{3 \delta+7}\right)$ & 7,8 \\
\hline 26) & $\left(\frac{2 h(\delta+1)}{3 \delta+7},-\frac{2 h(\delta+5)}{3 \delta+7}, \frac{2 h(\delta+1)}{3 \delta+7},-h \sqrt{-\frac{\delta+5}{3 \delta+7}}, \frac{h(\delta+5)}{3 \delta+7},-h \sqrt{-\frac{\delta+5}{3 \delta+7}}, \frac{h \sqrt{-(\delta-3)(\delta+5)}}{3 \delta+7},-h \sqrt{\frac{\delta-3}{3 \delta+7}},-\frac{h \sqrt{-(\delta-3)(\delta+5)}}{3 \delta+7}\right)$ & 7,8 \\
\hline 27) & $\left(\frac{2 h(\delta+1)}{3 \delta+7},-\frac{2 h(\delta+5)}{3 \delta+7}, \frac{2 h(\delta+1)}{3 \delta+7}, h \sqrt{-\frac{\delta+5}{3 \delta+7}},-\frac{h(\delta+5)}{3 \delta+7},-h \sqrt{-\frac{\delta+5}{3 \delta+7}},-\frac{h \sqrt{-(\delta-3)(\delta+5)}}{3 \delta+7}, h \sqrt{\frac{\delta-3}{3 \delta+7}},-\frac{h \sqrt{-(\delta-3)(\delta+5)}}{3 \delta+7}\right)$ & 7,8 \\
\hline 28) & $\left(\frac{2 h(\delta+1)}{3 \delta+7},-\frac{2 h(\delta+5)}{3 \delta+7}, \frac{2 h(\delta+1)}{3 \delta+7}, h \sqrt{-\frac{\delta+5}{3 \delta+7}},-\frac{h(\delta+5)}{3 \delta+7},-h \sqrt{-\frac{\delta+5}{3 \delta+7}}, \frac{h \sqrt{-(\delta-3)(\delta+5)}}{3 \delta+7},-h \sqrt{\frac{\delta-3}{3 \delta+7}}, \frac{h \sqrt{-(\delta-3)(\delta+5)}}{3 \delta+7}\right)$ & 7,8 \\
\hline 29) & $\left(\frac{2 h(\delta+1)}{3 \delta+7},-\frac{2 h(\delta+5)}{3 \delta+7}, \frac{2 h(\delta+1)}{3 \delta+7}, h \sqrt{-\frac{\delta+5}{3 \delta+7}}, \frac{h(\delta+5)}{3 \delta+7}, h \sqrt{-\frac{\delta+5}{3 \delta+7}},-\frac{h \sqrt{-(\delta-3)(\delta+5)}}{3 \delta+7},-h \sqrt{\frac{\delta-3}{3 \delta+7}}, \frac{h \sqrt{-(\delta-3)(\delta+5)}}{3 \delta+7}\right)$ & 7,8 \\
\hline & $\left(\frac{2 h(\delta+1)}{3 \delta+7},-\frac{2 h(\delta+5)}{3 \delta+7}, \frac{2 h(\delta+1)}{3 \delta+7}, h \sqrt{-\frac{\delta+5}{3 \delta+7}}, \frac{h(\delta+5)}{3 \delta+7}, h \sqrt{-\frac{\delta+5}{3 \delta+7}}, \frac{h \sqrt{-(\delta-3)(\delta+5)}}{3 \delta+7}, h \sqrt{\frac{\delta-3}{3 \delta+7}},-\frac{h \sqrt{-(\delta-3)(\delta+5)}}{3 \delta+7}\right)$ & 7,8 \\
\hline
\end{tabular}

TABLE 7 . Critical points of $\overline{\mathcal{H}}$ in $\mathcal{B}(h)$ for $h>0$. 


\begin{tabular}{|c|c|}
\hline Cr. point & $\left(U_{1}, \psi_{1}\right)$ \\
\hline$O_{1}^{-}$ & - \\
$O_{2}^{-}$ & $\left(-\sqrt{\frac{-2 h(\delta+9)}{3(\delta+5)}},-\sqrt{\frac{2 h(\delta+3)}{3(\delta+5)}}, 0,0\right)$ \\
$O_{3}^{-}$ & $\left(-\sqrt{\frac{-2 h(\delta+9)}{3(\delta+5)}}, \sqrt{\frac{2 h(\delta+3)}{3(\delta+5)}}, 0,0\right)$ \\
$O_{4}^{-}$ & $\left(\sqrt{\frac{-2 h(\delta+9)}{3(\delta+5)}},-\sqrt{\frac{2 h(\delta+3)}{3(\delta+5)}}, 0,0\right)$ \\
$O_{5}^{-}$ & $\left(\sqrt{\frac{-2 h(\delta+9)}{3(\delta+5)}}, \sqrt{\frac{2 h(\delta+3)}{3(\delta+5)}}, 0,0\right)$ \\
$O_{6}^{-}$ & $\left(0,-\sqrt{\frac{2 h(\delta+1)}{3 \delta+7}},-\sqrt{\frac{-2 h(\delta+5)}{3 \delta+7}}, 0\right)$ \\
$O_{7}^{-}$ & $\left(0,-\sqrt{\frac{2 h(\delta+1)}{3 \delta+7}}, \sqrt{\frac{-2 h(\delta+5)}{3 \delta+7}}, 0\right)$ \\
$O_{8}^{-}$ & $\left(0, \sqrt{\frac{2 h(\delta+1)}{3 \delta+7}},-\sqrt{\frac{-2 h(\delta+5)}{3 \delta+7}}, 0\right)$ \\
$O_{9}^{-}$ & $\left(0, \sqrt{\frac{2 h(\delta+1)}{3 \delta+7}}, \sqrt{\frac{-2 h(\delta+5)}{3 \delta+7}}, 0\right)$ \\
$O_{10}^{-}$ & $\left(\sqrt{\frac{-2 h(\delta-1)(\delta+5)}{3 \delta^{2}+6 \delta-13}}, 0,0,-\sqrt{\frac{2 h\left(\delta^{2}-9\right)}{3 \delta^{2}+6 \delta-13}}\right)$ \\
$O_{11}^{-}$ & $\left(\sqrt{\frac{-2 h(\delta-1)(\delta+5)}{3 \delta^{2}+6 \delta-13}}, 0,0, \sqrt{\frac{2 h\left(\delta^{2}-9\right)}{3 \delta^{2}+6 \delta-13}}\right)$ \\
$O_{12}^{-}$ & $\left(-\sqrt{\frac{-2 h(\delta-1)(\delta+5)}{3 \delta^{2}+6 \delta-13}}, 0,0,-\sqrt{\frac{2 h\left(\delta^{2}-9\right)}{3 \delta^{2}+6 \delta-13}}\right)$ \\
$O_{13}^{-}$ & $\left(-\sqrt{\frac{-2 h(\delta-1)(\delta+5)}{3 \delta^{2}+6 \delta-13}}, 0,0, \sqrt{\frac{2 h\left(\delta^{2}-9\right)}{3 \delta^{2}+6 \delta-13}}\right)$ \\
$O_{14}^{-}$ & $\left(0,0,-\sqrt{\frac{-2 h(\delta-1)(\delta+5)}{3 \delta^{2}+6 \delta-13}},(\delta+1) \sqrt{\frac{2 h}{3 \delta^{2}+6 \delta-13}}\right)$ \\
$O_{15}^{-}$ & $\left(0,0, \sqrt{\frac{-2 h(\delta-1)(\delta+5)}{3 \delta^{2}+6 \delta-13}},-(\delta+1) \sqrt{\frac{2 h}{3 \delta^{2}+6 \delta-13}}\right)$ \\
$O_{16}^{-}$ & $\left(0,0,-\sqrt{\frac{-2 h(\delta-1)(\delta+5)}{3 \delta^{2}+6 \delta-13}},-(\delta+1) \sqrt{\frac{2 h}{3 \delta^{2}+6 \delta-13}}\right)$ \\
$O_{17}^{-}$ & $\left(0,0, \sqrt{\frac{-2 h(\delta-1)(\delta+5)}{3 \delta^{2}+6 \delta-13}},(\delta+1) \sqrt{\frac{2 h}{3 \delta^{2}+6 \delta-13}}\right)$ \\
\hline
\end{tabular}

TABLE 8. Critical points of $\overline{\mathcal{H}}$ for $h<0$ in the local chart $\left(U_{1}, \psi_{1}\right): O_{1}^{-}$corresponds to point 1) of Table $6, O_{2}^{-}$to point 2$), \ldots, O_{17}^{-}$to point 17$)$. 


\begin{tabular}{|c|c|}
\hline Cr. point & $\left(U_{2}, \psi_{2}\right)$ \\
\hline$O_{1}^{-}$ & $(0,0,0,0)$ \\
\hline$O_{2}^{-}$ & $\left(-\sqrt{\frac{2 h(\delta+3)}{3(\delta+5)}}, \sqrt{\frac{2 h(\delta+3)}{3(\delta+5)}}, 0,0\right)$ \\
\hline$O_{3}^{-}$ & $\left(-\sqrt{\frac{2 h(\delta+3)}{3(\delta+5)}},-\sqrt{\frac{2 h(\delta+3)}{3(\delta+5)}}, 0\right)$ \\
\hline$O_{4}^{-}$ & $\left(\sqrt{\frac{2 h(\delta+3)}{3(\delta+5)}},-\sqrt{\frac{2 h(\delta+3)}{3(\delta+5)}}, 0,0\right)$ \\
\hline$O_{5}^{-}$ & $\left(\sqrt{\frac{2 h(\delta+3)}{3(\delta+5)}}, \sqrt{\frac{2 h(\delta+3)}{3(\delta+5)}}, 0,0\right)$ \\
\hline$O_{6}^{-}$ & $\left(0,0,-\sqrt{\frac{2 h(\delta+1)}{3 \delta+7}}, \sqrt{\frac{2 h(\delta+1)}{3 \delta+7}}\right)$ \\
\hline$O_{7}^{-}$ & $\left(0,0, \sqrt{\frac{2 h(\delta+1)}{3 \delta+7}},-\sqrt{\frac{2 h(\delta+1)}{3 \delta+7}}, 0\right)$ \\
\hline$O_{8}^{-}$ & $\left(0,0,-\sqrt{\frac{2 h(\delta+1)}{3 \delta+7}},-\sqrt{\frac{2 h(\delta+1)}{3 \delta+7}}, 0\right)$ \\
\hline$O_{9}^{-}$ & $\left(0,0, \sqrt{\frac{2 h(\delta+1)}{3 \delta+7}}, \sqrt{\frac{2 h(\delta+1)}{3 \delta+7}}\right)$ \\
\hline$O_{10}^{-}$ & $\left(-(\delta+1) \sqrt{\frac{2 h}{3 \delta^{2}+6 \delta-13}}, 0,0,-\sqrt{\frac{2 h\left(\delta^{2}-9\right)}{3 \delta^{2}+6 \delta-13}}\right)$ \\
\hline$O_{11}^{-}$ & $\left(-(\delta+1) \sqrt{\frac{2 h}{3 \delta^{2}+6 \delta-13}}, 0,0, \sqrt{\frac{2 h\left(\delta^{2}-9\right)}{3 \delta^{2}+6 \delta-13}}\right)$ \\
\hline$O_{12}^{-}$ & $\left((\delta+1) \sqrt{\frac{2 h}{3 \delta^{2}+6 \delta-13}}, 0,0, \sqrt{\frac{2 h\left(\delta^{2}-9\right)}{3 \delta^{2}+6 \delta-13}}\right)$ \\
\hline$O_{13}^{-}$ & $\left((\delta+1) \sqrt{\frac{2 h}{3 \delta^{2}+6 \delta-13}}, 0,0,-\sqrt{\frac{2 h\left(\delta^{2}-9\right)}{3 \delta^{2}+6 \delta-13}}\right)$ \\
\hline$O_{14}^{-}$ & $\left(0,(\delta+1) \sqrt{\frac{2 h}{3 \delta^{2}+6 \delta-13}},-\sqrt{\frac{2 h\left(\delta^{2}-9\right)}{3 \delta^{2}+6 \delta-13}}, 0\right)$ \\
\hline$O_{15}^{-}$ & $\left(0,(\delta+1) \sqrt{\frac{2 h}{3 \delta^{2}+6 \delta-13}}, \sqrt{\frac{2 h\left(\delta^{2}-9\right)}{3 \delta^{2}+6 \delta-13}}, 0\right)$ \\
\hline$O_{16}^{-}$ & $\left(0,-(\delta+1) \sqrt{\frac{2 h}{3 \delta^{2}+6 \delta-13}},-\sqrt{\frac{2 h\left(\delta^{2}-9\right)}{3 \delta^{2}+6 \delta-13}}, 0\right)$ \\
\hline$O_{17}^{-}$ & $\left(0,-(\delta+1) \sqrt{\frac{2 h}{3 \delta^{2}+6 \delta-13}}, \sqrt{\frac{2 h\left(\delta^{2}-9\right)}{3 \delta^{2}+6 \delta-13}}, 0\right)$ \\
\hline
\end{tabular}

TABLE 9. Critical points of $\overline{\mathcal{H}}$ for $h<0$ in the local chart $\left(U_{2}, \psi_{2}\right): O_{1}^{-}$corresponds to point 1) of Table $6, O_{2}^{-}$to point 2$), \ldots, O_{17}^{-}$to point 17$)$. 


\begin{tabular}{|c|c|}
\hline Cr. point & $\left(U_{3}, \psi_{3}\right)$ \\
\hline$O_{1}^{-}$ & - \\
\hline $\mathrm{O}_{2}^{-}$ & $\left(-\sqrt{\frac{2 h(\delta+3)}{3(\delta+5)}}, \sqrt{\frac{-2 h(\delta+9)}{3(\delta+5)}}, 0,0\right)$ \\
\hline$O_{3}^{-}$ & $\left(\sqrt{\frac{2 h(\delta+3)}{3(\delta+5)}},-\sqrt{\frac{-2 h(\delta+9)}{3(\delta+5)}}, 0,0\right)$ \\
\hline$O_{4}^{-}$ & $\left(-\sqrt{\frac{2 h(\delta+3)}{3(\delta+5)}},-\sqrt{\frac{-2 h(\delta+9)}{3(\delta+5)}}, 0,0\right)$ \\
\hline$O_{5}^{-}$ & $\left(\sqrt{\frac{2 h(\delta+3)}{3(\delta+5)}}, \sqrt{\frac{-2 h(\delta+9)}{3(\delta+5)}}, 0,0\right)$ \\
\hline$O_{6}^{-}$ & $\left(-\sqrt{\frac{2 h(\delta+1)}{3 \delta+7}}, 0,0, \sqrt{\frac{-2 h(\delta+5)}{3 \delta+7}}\right)$ \\
\hline$O_{7}^{-}$ & $\left(-\sqrt{\frac{2 h(\delta+1)}{3 \delta+7}}, 0,0\right.$ \\
\hline$O_{8}^{-}$ & $\left(\sqrt{\frac{2 h(\delta+1)}{3 \delta+7}}, 0,0,-\right.$ \\
\hline$O_{9}^{-}$ & $\left(\sqrt{\frac{2 h(\delta+1)}{3 \delta+7}}, 0,0\right.$ \\
\hline$O_{10}^{-}$ & + 1) $\left.\sqrt{\frac{2 h}{3 \delta^{2}+6 \delta-13}},-\sqrt{\frac{-2 h(\delta-1)(\delta+5)}{3 \delta^{2}+6 \delta-13}}\right)$ \\
\hline$O_{11}^{-}$ & $\left(0,0,(\delta+1) \sqrt{\frac{2 h}{3 \delta^{2}+6 \delta-13}}, \sqrt{\frac{-2 h(\delta-1)(\delta+5)}{3 \delta^{2}+6 \delta-13}}\right)$ \\
\hline$O_{12}^{-}$ & $\left(0,0,-(\delta+1) \sqrt{\frac{h}{3 \delta^{2}+6 \delta-13}}, \sqrt{\frac{-2 h(\delta-1)(\delta+5)}{3 \delta^{2}+6 \delta-13}}\right)$ \\
\hline$O_{13}^{-}$ & $\left(0,0,(\delta+1) \sqrt{\frac{h}{3 \delta^{2}+6 \delta-13}},-\sqrt{\frac{-2 h(\delta-1)(\delta+5)}{3 \delta^{2}+6 \delta-13}}\right)$ \\
\hline$O_{14}^{-}$ & $\left(0,-\sqrt{\frac{-2 h(\delta-1)(\delta+5)}{3 \delta^{2}+6 \delta-13}}, \sqrt{\frac{2 h\left(\delta^{2}-9\right)}{3 \delta^{2}+6 \delta-13}}, 0\right)$ \\
\hline$O_{15}^{-}$ & $\left(0,-\sqrt{\frac{-2 h(\delta-1)(\delta+5)}{3 \delta^{2}+6 \delta-13}},-\sqrt{\frac{2 h\left(\delta^{2}-9\right)}{3 \delta^{2}+6 \delta-13}}, 0\right)$ \\
\hline$O_{16}^{-}$ & $\left(0, \sqrt{\frac{-2 h(\delta-1)(\delta+5)}{3 \delta^{2}+6 \delta-13}},-\sqrt{\frac{2 h\left(\delta^{2}-9\right)}{3 \delta^{2}+6 \delta-13}}, 0\right)$ \\
\hline$O_{17}^{-}$ & $\left(0, \sqrt{\frac{-2 h(\delta-1)(\delta+5)}{3 \delta^{2}+6 \delta-13}}, \sqrt{\frac{2 h\left(\delta^{2}-9\right)}{3 \delta^{2}+6 \delta-13}}, 0\right)$ \\
\hline
\end{tabular}

TABLE 10. Critical points of $\overline{\mathcal{H}}$ for $h<0$ in the local chart $\left(U_{3}, \psi_{3}\right): O_{1}^{-}$corresponds to point 1) of Table $6, O_{2}^{-}$to point 2$), \ldots, O_{17}^{-}$to point 17 ). 


\begin{tabular}{|c|c|}
\hline Cr. point & $\left(U_{1}, \psi_{1}\right)$ \\
\hline$O_{1}^{+}$ & $(0,0,0,0)$ \\
\hline $\mathrm{O}_{2}^{+}$ & - \\
\hline$O_{3}^{+}$ & $(0,0,0,-\sqrt{h})$ \\
\hline$O_{4}^{+}$ & $(0,0,0, \sqrt{h})$ \\
\hline$O_{5}^{+}$ & $(0,-\sqrt{h}, 0,0)$ \\
\hline$O_{6}^{+}$ & $(0, \sqrt{h}, 0,0)$ \\
\hline$O_{7}^{+}$ & $\left(\sqrt{\frac{-2 h(\delta+9)}{3(\delta+5)}},-\sqrt{\frac{2 h(\delta+3)}{3(\delta+5)}}, 0,0\right)$ \\
\hline $\mathrm{O}_{8}^{+}$ & $\left(\sqrt{\frac{-2 h(\delta+9)}{3(\delta+5)}}, \sqrt{\frac{2 h(\delta+3)}{3(\delta+5)}}, 0,0\right)$ \\
\hline $\mathrm{O}_{9}^{+}$ & $\left(-\sqrt{\frac{-2 h(\delta+9)}{3(\delta+5)}},-\sqrt{\frac{2 h(\delta+3)}{3(\delta+5)}}, 0,0\right)$ \\
\hline$O_{10}^{+}$ & $\left(-\sqrt{\frac{-2 h(\delta+9)}{3(\delta+5)}}, \sqrt{\frac{2 h(\delta+3)}{3(\delta+5)}}, 0,0\right)$ \\
\hline$O_{11}^{+}$ & $\left(0,-\sqrt{\frac{2 h(\delta+1)}{3 \delta+7}}, \sqrt{\frac{-2 h(\delta+5)}{3 \delta+7}}, 0\right)$ \\
\hline$O_{12}^{+}$ & $\left(0,-\sqrt{\frac{2 h(\delta+1)}{3 \delta+7}},-\sqrt{\frac{-2 h(\delta+5)}{3 \delta+7}}, 0\right)$ \\
\hline$O_{13}^{+}$ & $\left(0, \sqrt{\frac{2 h(\delta+1)}{3 \delta+7}}, \sqrt{\frac{-2 h(\delta+5)}{3 \delta+7}}, 0\right)$ \\
\hline$O_{14}^{+}$ & $\left(0, \sqrt{\frac{2 h(\delta+1)}{3 \delta+7}},-\sqrt{\frac{-2 h(\delta+5)}{3 \delta+7}}, 0\right)$ \\
\hline$O_{15}^{+}$ & $\left.\frac{(\delta-1)(\delta+5)}{2+6 \delta-13}, 0,0, \sqrt{\frac{2 h\left(\delta^{2}-9\right)}{3 \delta^{2}+6 \delta-13}}\right)$ \\
\hline$O_{16}^{+}$ & $\left.\frac{-1)(\delta+5)}{-66 \delta-13}, 0,0,-\sqrt{\frac{2 h\left(\delta^{2}-9\right)}{3 \delta^{2}+6 \delta-13}}\right)$ \\
\hline$O_{17}^{+}$ & $\left(\sqrt{\frac{-2 h(\delta-1)(\delta+5)}{3 \delta^{2}+6 \delta-13}}, 0,0, \sqrt{\frac{2 h\left(\delta^{2}-9\right)}{3 \delta^{2}+6 \delta-13}}\right)$ \\
\hline$O_{18}^{+}$ & $\left(\sqrt{\frac{-2 h(\delta-1)(\delta+5)}{3 \delta^{2}+6 \delta-13}}, 0,0,-\sqrt{\frac{2 h\left(\delta^{2}-9\right)}{3 \delta^{2}+6 \delta-13}}\right)$ \\
\hline$O_{19}^{+}$ & $\left(0,0, \sqrt{\frac{-2 h(\delta-1)(\delta+5)}{3 \delta^{2}+6 \delta-13}},-(\delta+1) \sqrt{\frac{2 h}{3 \delta^{2}+6 \delta-13}}\right)$ \\
\hline$O_{20}^{+}$ & $\left(0,0,-\sqrt{\frac{-2 h(\delta-1)(\delta+5)}{3 \delta^{2}+6 \delta-13}},(\delta+1) \sqrt{\frac{2 h}{3 \delta^{2}+6 \delta-13}}\right)$ \\
\hline$O_{21}^{+}$ & $\left(0,0, \sqrt{\frac{-2 h(\delta-1)(\delta+5)}{3 \delta^{2}+6 \delta-13}},(\delta+1) \sqrt{\frac{2 h}{3 \delta^{2}+6 \delta-13}}\right)$ \\
\hline $\mathrm{O}_{22}^{+}$ & $\left(0,0,-\sqrt{\frac{-2 h(\delta-1)(\delta+5)}{3 \delta^{2}+6 \delta-13}},-(\delta+1) \sqrt{\frac{2 h}{3 \delta^{2}+6 \delta-13}}\right)$ \\
\hline$O_{23}^{+}$ & $\left(-\sqrt{\frac{-h(\delta+5)}{2(\delta+1)}},(\delta+5) \sqrt{\frac{h}{2(\delta+1)(3 \delta+7)}}, \sqrt{\frac{-h(\delta-3)(\delta+5)}{2(\delta+1)(3 \delta+7)}},-\sqrt{\frac{h(\delta-3)}{2(\delta+1)}}\right)$ \\
\hline$O_{24}^{+}$ & $\left(-\sqrt{\frac{-h(\delta+5)}{2(\delta+1)}},(\delta+5) \sqrt{\frac{h}{2(\delta+1)(3 \delta+7)}},-\sqrt{\frac{-h(\delta-3)(\delta+5)}{2(\delta+1)(3 \delta+7)}}, \sqrt{\frac{h(\delta-3)}{2(\delta+1)}}\right)$ \\
\hline$O_{25}^{+}$ & $\left(-\sqrt{\frac{-h(\delta+5)}{2(\delta+1)}},-(\delta+5) \sqrt{\frac{h}{2(\delta+1)(3 \delta+7)}}, \sqrt{\frac{-h(\delta-3)(\delta+5)}{2(\delta+1)(3 \delta+7)}}, \sqrt{\frac{h(\delta-3)}{2(\delta+1)}}\right)$ \\
\hline$O_{26}^{+}$ & $\left(-\sqrt{\frac{-h(\delta+5)}{2(\delta+1)}},-(\delta+5) \sqrt{\frac{h}{2(\delta+1)(3 \delta+7)}},-\sqrt{\frac{-h(\delta-3)(\delta+5)}{2(\delta+1)(3 \delta+7)}},-\sqrt{\frac{h(\delta-3)}{2(\delta+1)}}\right)$ \\
\hline$O_{27}^{+}$ & $\left(\sqrt{\frac{-h(\delta+5)}{2(\delta+1)}},(\delta+5) \sqrt{\frac{h}{2(\delta+1)(3 \delta+7)}}, \sqrt{\frac{-h(\delta-3)(\delta+5)}{2(\delta+1)(3 \delta+7)}}, \sqrt{\frac{h(\delta-3)}{2(\delta+1)}}\right)$ \\
\hline$O_{28}^{+}$ & $\left(\sqrt{\frac{-h(\delta+5)}{2(\delta+1)}},(\delta+5) \sqrt{\frac{h}{2(\delta+1)(3 \delta+7)}},-\sqrt{\frac{-h(\delta-3)(\delta+5)}{2(\delta+1)(3 \delta+7)}},-\sqrt{\frac{h(\delta-3)}{2(\delta+1)}}\right)$ \\
\hline $\mathrm{O}_{29}^{+}$ & $\left(\sqrt{\frac{-h(\delta+5)}{2(\delta+1)}},-(\delta+5) \sqrt{\frac{h}{2(\delta+1)(3 \delta+7)}}, \sqrt{\frac{-h(\delta-3)(\delta+5)}{2(\delta+1)(3 \delta+7)}},-\sqrt{\frac{h(\delta-3)}{2(\delta+1)}}\right)$ \\
\hline$O_{30}^{+}$ & $\left(\sqrt{\frac{-h(\delta+5)}{2(\delta+1)}},-(\delta+5) \sqrt{\frac{h}{2(\delta+1)(3 \delta+7)}},-\sqrt{\frac{-h(\delta-3)(\delta+5)}{2(\delta+1)(3 \delta+7)}}, \sqrt{\frac{h(\delta-3)}{2(\delta+1)}}\right)$ \\
\hline
\end{tabular}

TABLE 11. Critical points of $\overline{\mathcal{H}}$ for $h>0$ in the local chart $\left(U_{1}, \psi_{1}\right): O_{1}^{+}$corresponds to point 1) of Table $7, \mathrm{O}_{2}^{+}$to point 2 ), .., $\mathrm{O}_{30}^{+}$to point 30 ). 


\begin{tabular}{|c|c|}
\hline Cr. point & $\left(U_{2}, \psi_{2}\right)$ \\
\hline$O_{1}^{+}$ & - \\
\hline $\mathrm{O}_{2}^{+}$ & - \\
\hline$O_{3}^{+}$ & - \\
\hline$O_{4}^{+}$ & - \\
\hline$O_{5}^{+}$ & - \\
\hline$O_{6}^{+}$ & - \\
\hline$O_{7}^{+}$ & $\left(\sqrt{\frac{2 h(\delta+3)}{3(\delta+5)}},-\sqrt{\frac{2 h(\delta+3)}{3(\delta+5)}}, 0,0\right)$ \\
\hline$O_{8}^{+}$ & $\left(\sqrt{\frac{2 h(\delta+3)}{3(\delta+5)}}, \sqrt{\frac{2 h(\delta+3)}{3(\delta+5)}}, 0,0\right)$ \\
\hline$O_{9}^{+}$ & $\left(-\sqrt{\frac{2 h(\delta+3)}{3(\delta+5)}}, \sqrt{\frac{2 h(\delta+3)}{3(\delta+5)}}, 0,0\right)$ \\
\hline$O_{10}^{+}$ & $\left(-\sqrt{\frac{2 h(\delta+3)}{3(\delta+5)}},-\sqrt{\frac{2 h(\delta+3)}{3(\delta+5)}}, 0,0\right)$ \\
\hline$O_{11}^{+}$ & $\left(0,0, \sqrt{\frac{2 h(\delta+1)}{3 \delta+7}},-\sqrt{\frac{2 h(\delta+1)}{3 \delta+7}}\right)$ \\
\hline$O_{12}^{+}$ & $\left(0,0,-\sqrt{\frac{2 h(\delta+1)}{3 \delta+7}}, \sqrt{\frac{2 h(\delta+1)}{3 \delta+7}}\right)$ \\
\hline$O_{13}^{+}$ & $\left(0,0, \sqrt{\frac{2 h(\delta+1)}{3 \delta+7}}, \sqrt{\frac{2 h(\delta+1)}{3 \delta+7}}\right)$ \\
\hline$O_{14}^{+}$ & $\left(0,0,-\sqrt{\frac{2 h(\delta+1)}{3 \delta+7}},-\sqrt{\frac{2 h(\delta+1)}{3 \delta+7}}\right)$ \\
\hline$O_{15}^{+}$ & $\left((\delta+1) \sqrt{\frac{2 h}{3 \delta^{2}+6 \delta-13}}, 0,0,-\sqrt{\frac{2 h\left(\delta^{2}-9\right)}{3 \delta^{2}+6 \delta-13}}\right)$ \\
\hline$O_{16}^{+}$ & $\left.\frac{2 h}{{ }^{2}+6 \delta-13}, 0,0, \sqrt{\frac{2 h\left(\delta^{2}-9\right)}{3 \delta^{2}+6 \delta-13}}\right)$ \\
\hline$O_{17}^{+}$ & $\left(-(\delta+1) \sqrt{\frac{2 h}{3 \delta^{2}+6 \delta-13}}, 0,0, \sqrt{\frac{2 h\left(\delta^{2}-9\right)}{3 \delta^{2}+6 \delta-13}}\right)$ \\
\hline$O_{18}^{+}$ & $\left.\frac{2 h}{+6 \delta-13}, 0,0,-\sqrt{\frac{2 h\left(\delta^{2}-9\right)}{3 \delta^{2}+6 \delta-13}}\right)$ \\
\hline$O_{19}^{+}$ & $\left(0,(\delta+1) \sqrt{\frac{2 h}{3 \delta^{2}+6 \delta-13}}, \sqrt{\frac{2 h\left(\delta^{2}-9\right)}{3 \delta^{2}+6 \delta-13}}, 0\right)$ \\
\hline$O_{20}^{+}$ & $\left(0,(\delta+1) \sqrt{\frac{2 h}{3 \delta^{2}+6 \delta-13}},-\sqrt{\frac{2 h\left(\delta^{2}-9\right)}{3 \delta^{2}+6 \delta-13}}, 0\right)$ \\
\hline$O_{21}^{+}$ & $\left(0,-(\delta+1) \sqrt{\frac{2 h}{3 \delta^{2}+6 \delta-13}}, \sqrt{\frac{2 h\left(\delta^{2}-9\right)}{3 \delta^{2}+6 \delta-13}}, 0\right)$ \\
\hline $\mathrm{O}_{22}^{+}$ & $\left(0,-(\delta+1) \sqrt{\frac{2 h}{3 \delta^{2}+6 \delta-13}},-\sqrt{\frac{2 h\left(\delta^{2}-9\right)}{3 \delta^{2}+6 \delta-13}}, 0\right)$ \\
\hline$O_{23}^{+}$ & $\left.\frac{h(\delta-3)}{(3 \delta+7)}, \sqrt{\frac{h(\delta-3)}{2(3 \delta+7)}}\right)$ \\
\hline$O_{24}^{+}$ & $\left.\sqrt{\frac{h(\delta-3)}{2(3 \delta+7)}}\right)$ \\
\hline$O_{25}^{+}$ & $\left.\sqrt{\frac{h}{2}}, \sqrt{\frac{h(\delta-3)}{2(3 \delta+7)}},-\sqrt{\frac{h(\delta-3)}{2(3 \delta+7)}}\right)$ \\
\hline $\mathrm{O}_{26}^{+}$ & $\left.\sqrt{\frac{h}{2}},-\sqrt{\frac{h(\delta-3)}{2(3 \delta+7)}}, \sqrt{\frac{h(\delta-3)}{2(3 \delta+7)}}\right)$ \\
\hline$O_{27}^{+}$ & $\left(\sqrt{\frac{h}{2}},-\sqrt{\frac{h}{2}}, \sqrt{\frac{h(\delta-3)}{2(3 \delta+7)}}, \sqrt{\frac{h(\delta-3)}{2(3 \delta+7)}}\right)$ \\
\hline$O_{28}^{+}$ & $\left(\sqrt{\frac{h}{2}},-\sqrt{\frac{h}{2}},-\sqrt{\frac{h(\delta-3)}{2(3 \delta+7)}},-\sqrt{\frac{h(\delta-3)}{2(3 \delta+7)}}\right)$ \\
\hline$O_{29}^{+}$ & $\left(\sqrt{\frac{h}{2}}, \sqrt{\frac{h}{2}}, \sqrt{\frac{h(\delta-3)}{2(3 \delta+7)}},-\sqrt{\frac{h(\delta-3)}{2(3 \delta+7)}}\right)$ \\
\hline$O_{30}^{+}$ & $\left(\sqrt{\frac{h}{2}}, \sqrt{\frac{h}{2}},-\sqrt{\frac{h(\delta-3)}{2(3 \delta+7)}}, \sqrt{\frac{h(\delta-3)}{2(3 \delta+7)}}\right)$ \\
\hline
\end{tabular}

TABLE 12. Critical points of $\overline{\mathcal{H}}$ for $h>0$ in the local chart $\left(U_{2}, \psi_{2}\right): O_{1}^{+}$corresponds to point 1) of Table $7, \mathrm{O}_{2}^{+}$to point 2$), \ldots, \mathrm{O}_{30}^{+}$to point 30 ). 


\begin{tabular}{|c|c|}
\hline Cr. point & $\left(U_{3}, \psi_{3}\right)$ \\
\hline$O_{1}^{+}$ & - \\
\hline $\mathrm{O}_{2}^{+}$ & $(0,0,0,0)$ \\
\hline$O_{3}^{+}$ & $(0,0, \sqrt{h}, 0)$ \\
\hline$O_{4}^{+}$ & $(0,0,-\sqrt{h}, 0)$ \\
\hline$O_{5}^{+}$ & $(-\sqrt{h}, 0,0,0)$ \\
\hline$O_{6}^{+}$ & $(\sqrt{h}, 0,0,0)$ \\
\hline$O_{7}^{+}$ & $\left(-\sqrt{\frac{2 h(\delta+3)}{3(\delta+5)}},-\sqrt{\frac{-2 h(\delta+9)}{3(\delta+5)}}, 0,0\right)$ \\
\hline$O_{8}^{+}$ & $\left(\sqrt{\frac{2 h(\delta+3)}{3(\delta+5)}}, \sqrt{\frac{-2 h(\delta+9)}{3(\delta+5)}}, 0,0\right)$ \\
\hline$O_{9}^{+}$ & $\left(-\sqrt{\frac{2 h(\delta+3)}{3(\delta+5)}}, \sqrt{\frac{-2 h(\delta+9)}{3(\delta+5)}}, 0,0\right)$ \\
\hline$O_{10}^{+}$ & $\left(\sqrt{\frac{2 h(\delta+3)}{3(\delta+5)}},-\sqrt{\frac{-2 h(\delta+9)}{3(\delta+5)}}, 0,0\right)$ \\
\hline$O_{11}^{+}$ & $\left(-\sqrt{\frac{2 h(\delta+1)}{3 \delta+7}}, 0,0,-\sqrt{\frac{-2 h(\delta+5)}{3 \delta+7}}\right)$ \\
\hline$O_{12}^{+}$ & $-\sqrt{\frac{2 h(\delta+1)}{3 \delta+7}}, 0,0$ \\
\hline$O_{13}^{+}$ & $\left.\frac{1}{1)}, 0,0, \sqrt{\frac{-2 h(\delta+5)}{3 \delta+7}}\right)$ \\
\hline$O_{14}^{+}$ & $\left.\frac{-2 h(\delta+5)}{3 \delta+7}\right)$ \\
\hline$O_{15}^{+}$ & $\left(0,0,(\delta+1) \sqrt{\frac{2 h}{3 \delta^{2}+6 \delta-13}},-\sqrt{\frac{-2 h(\delta-1)(\delta+5)}{3 \delta^{2}+6 \delta-13}}\right)$ \\
\hline$O_{16}^{+}$ & $\left(0,0,-(\delta+1) \sqrt{\frac{2 h}{3 \delta^{2}+6 \delta-13}}, \sqrt{\frac{-2 h(\delta-1)(\delta+5)}{3 \delta^{2}+6 \delta-13}}\right)$ \\
\hline$O_{17}^{+}$ & $\left(0,0,(\delta+1) \sqrt{\frac{2 h}{3 \delta^{2}+6 \delta-13}}, \sqrt{\frac{-2 h(\delta-1)(\delta+5)}{3 \delta^{2}+6 \delta-13}}\right)$ \\
\hline$O_{18}^{+}$ & $\left(0,0,-(\delta+1) \sqrt{\frac{2 h}{3 \delta^{2}+6 \delta-13}},-\sqrt{\frac{-2 h(\delta-1)(\delta+5)}{3 \delta^{2}+6 \delta-13}}\right)$ \\
\hline$O_{19}^{+}$ & $\left(0,-\sqrt{\frac{-2 h(\delta-1)(\delta+5)}{3 \delta^{2}+6 \delta-13}},-\sqrt{\frac{2 h\left(\delta^{2}-9\right)}{3 \delta^{2}+6 \delta-13}}, 0\right)$ \\
\hline $\mathrm{O}_{20}^{+}$ & $\left(0,-\sqrt{\frac{-2 h(\delta-1)(\delta+5)}{3 \delta^{2}+6 \delta-13}}, \sqrt{\frac{2 h\left(\delta^{2}-9\right)}{3 \delta^{2}+6 \delta-13}}, 0\right)$ \\
\hline$O_{21}^{+}$ & $\left(0, \sqrt{\frac{-2 h(\delta-1)(\delta+5)}{3 \delta^{2}+6 \delta-13}}, \sqrt{\frac{2 h\left(\delta^{2}-9\right)}{3 \delta^{2}+6 \delta-13}}, 0\right)$ \\
\hline$O_{22}^{+}$ & $\left(0, \sqrt{\frac{-2 h(\delta-1)(\delta+5)}{3 \delta^{2}+6 \delta-13}},-\sqrt{\frac{2 h\left(\delta^{2}-9\right)}{3 \delta^{2}+6 \delta-13}}, 0\right)$ \\
\hline$O_{23}^{+}$ & $\left((\delta+5) \sqrt{\frac{h}{2(\delta+1)(3 \delta+7)}}, \sqrt{\frac{-h(\delta+5)}{2(\delta+1)}}, \sqrt{\frac{h(\delta-3)}{2(\delta+1)}}, \sqrt{\frac{-h(\delta-3)(\delta+5)}{2(\delta+1)(3 \delta+7)}}\right)$ \\
\hline$O_{24}^{+}$ & $\left((\delta+5) \sqrt{\frac{h}{2(\delta+1)(3 \delta+7)}}, \sqrt{\frac{-h(\delta+5)}{2(\delta+1)}},-\sqrt{\frac{h(\delta-3)}{2(\delta+1)}},-\sqrt{\frac{-h(\delta-3)(\delta+5)}{2(\delta+1)(3 \delta+7)}}\right)$ \\
\hline$O_{25}^{+}$ & $\left(-(\delta+5) \sqrt{\frac{h}{2(\delta+1)(3 \delta+7)}},-\sqrt{\frac{-h(\delta+5)}{2(\delta+1)}},-\sqrt{\frac{h(\delta-3)}{2(\delta+1)}},-\sqrt{\frac{-h(\delta-3)(\delta+5)}{2(\delta+1)(3 \delta+7)}}\right)$ \\
\hline$O_{26}^{+}$ & $\left(-(\delta+5) \sqrt{\frac{h}{2(\delta+1)(3 \delta+7)}},-\sqrt{\frac{-h(\delta+5)}{2(\delta+1)}}, \sqrt{\frac{h(\delta-3)}{2(\delta+1)}}, \sqrt{\frac{-h(\delta-3)(\delta+5)}{2(\delta+1)(3 \delta+7)}}\right)$ \\
\hline$O_{27}^{+}$ & $\left((\delta+5) \sqrt{\frac{h}{2(\delta+1)(3 \delta+7)}},-\sqrt{\frac{-h(\delta+5)}{2(\delta+1)}},-\sqrt{\frac{h(\delta-3)}{2(\delta+1)}}, \sqrt{\frac{-h(\delta-3)(\delta+5)}{2(\delta+1)(3 \delta+7)}}\right)$ \\
\hline$O_{28}^{+}$ & $\left((\delta+5) \sqrt{\frac{h}{2(\delta+1)(3 \delta+7)}},-\sqrt{\frac{-h(\delta+5)}{2(\delta+1)}}, \sqrt{\frac{h(\delta-3)}{2(\delta+1)}},-\sqrt{\frac{-h(\delta-3)(\delta+5)}{2(\delta+1)(3 \delta+7)}}\right)$ \\
\hline $\mathrm{O}_{29}^{+}$ & $\left(-(\delta+5) \sqrt{\frac{h}{2(\delta+1)(3 \delta+7)}}, \sqrt{\frac{-h(\delta+5)}{2(\delta+1)}}, \sqrt{\frac{h(\delta-3)}{2(\delta+1)}},-\sqrt{\frac{-h(\delta-3)(\delta+5)}{2(\delta+1)(3 \delta+7)}}\right)$ \\
\hline$O_{30}^{+}$ & $\left(-(\delta+5) \sqrt{\frac{h}{2(\delta+1)(3 \delta+7)}}, \sqrt{\frac{-h(\delta+5)}{2(\delta+1)}},-\sqrt{\frac{h(\delta-3)}{2(\delta+1)}}, \sqrt{\frac{-h(\delta-3)(\delta+5)}{2(\delta+1)(3 \delta+7)}}\right)$ \\
\hline
\end{tabular}

TABLE 13. Critical points of $\overline{\mathcal{H}}$ for $h>0$ in the local chart $\left(U_{3}, \psi_{3}\right): O_{1}^{+}$corresponds to point 1) of Table $7, O_{2}^{+}$to point 2 ), .., $O_{30}^{+}$to point 30 ). 


\section{ACKNOWLEDGEMENTS}

We would like to thank the referees by carefully reading the paper and making constructive suggestions to get a better version of it.

\section{REFERENCES}

[1] V.I. Arnold, V.V. Kozlov, and A.I. Neishtadt, Mathematical Aspects of Classical and Celestial Mechanics, Encyclopaedia of Mathematical Sciences Vol. 3, Dynamical Systems III - Third Edition. Springer-Verlag, Berlin (2006).

[2] H.W. Broer, H. Hanssmann, And J. You, Bifurcations of normally parabolic tori in Hamiltonian systems, Nonlinearity 18 (2005), 1735-1769.

[3] E. Calzetta and C. El Hasi, Chaotic Friedmann-Robertson-Walker cosmology, Class. Quantum Grav. 10 (1993), 1825-1841.

[4] D. Carrasco, J.F. Palacián, C. Vidal, J. Vidarte, and P. Yanguas, Dynamics of axially symmetric perturbed Hamiltonians in 1:1:1 resonance, J. Nonlinear Sci. 28 (2018), 1293-1359.

[5] R.C. Churchill, M. Kummer, And D.L. Rod, On averaging, reduction, and symmetry in Hamiltonian systems, J. Differential Equations 49 (1983), 359-414.

[6] R.H. Cushman and L.M. Bates, Global Aspects of Classical Integrable Systems, Second Edition. Birkhäuser Verlag, Basel (2015).

[7] A. Deprit, Canonical transformations depending on a small parameter, Celestial Mech. 1 (1969), 12-30.

[8] A. Deprit, Delaunay normalisations, Celestial Mech. 26 (1982), 9-21.

[9] A. Deprit, The Lissajous transformation I. Basics, Celestial Mech. 51 (1991), 201-225.

[10] K. Efstathiou And D.A. Sadovskí, Perturbations of the 1:1:1 resonance with tetrahedral symmetry: a three degree of freedom analogue of the two degree of freedom Hénon-Heiles Hamiltonian, Nonlinearity 17 (2004), 415-446.

[11] K. Efstathiou, D.A. Sadovskí, and R.H. Cushman, Linear Hamiltonian Hopf bifurcation for point-groupinvariant perturbations of the 1:1:1 resonance, Proc. R. Soc. Lond. A 459 (2003), 2997-3019.

[12] M.M. El-Dessoky, A.A. Elmandouh, And A. Hobiny, Periodic orbits of the generalized FriedmannRobertson-Walker potential in galactic dynamics in a rotating reference frame, AIP Advances 7 (2017), 035021$1-8$.

[13] S. Ferrer, H. Hanssmann, J.F. Palacián, and P. Yanguas, On perturbed oscillators in 1-1-1 resonance: the case of axially symmetric cubic potentials, J. Geom. Phys. 40 (2002), 320-369.

[14] S. Ferrer, J.F. Palacián, And P. Yanguas, Hamiltonian oscillators in 1-1-1 resonance: normalization and integrability, J. Nonlinear Sci. 10 (2000), 145-174.

[15] A. Friedmann, Über die Krümmung des Raumes, Zeitschrift für Physik A 10 (1922), 377-386.

[16] A. Friedmann, Über die Möglichkeit einer Welt mit konstanter negativer Krümmung des Raumes, Zeitschrift für Physik A 21 (1922), 326-332.

[17] Y. Han, Y. LI, AND Y. YI, Invariant tori in Hamiltonian systems with high order proper degeneracy, Ann. Henri Poincaré 10 (2010), 1419-1436.

[18] H. Hanssmann, Local and Semi-Local Bifurcations in Hamiltonian Dynamical Systems: Results and Examples, Lectures Notes in Math. 1893. Springer-Verlag, New York (2007).

[19] H. Hanssmann And J.-C. VAn DeR MeER, Algebraic methods for determining Hamiltonian Hopf bifurcations in three-degree-of-freedom systems, J. Dynam. Differential Equations 17 (2005), 455-474.

[20] S.W. Hawking, The quantum state of the universe, Nuclear Physics B 239 (1984), 257-276.

[21] S.W. Hawking, Quantum cosmology, in Relativity, Groups and Topology II, edited by B.S. DeWitt and R. Stora (North-Holland, Amsterdam, 1984), pp. 333-379.

[22] S.W. Hawking, Arrow of time in cosmology, Phys. Rev. D 32 (1985), 2489-2495.

[23] G. Lemaître, Un univers homogène de masse constante et de rayon croissant, rendant compte de la vitesse radiale des nébuleuses extra-galactiques, Annales de la Société Scientifique de Bruxelles A47 (1927), 49-59.

[24] G. Lemaître, L'univers en expansion, Annales de la Société Scientifique de Bruxelles A53 (1933), 51-85.

[25] F.E. Lembarki And J. Llibre, Periodic orbits for a generalized Friedmann-Robertson-Walker Hamiltonian systems in dimension 6, Discrete Cont. Dyn.-S 8 (2015) 1165-1211.

[26] J. Llibre AND A. Makhlouf, Periodic orbits of the generalized Friedmann-Robertson-Walker Hamiltonian systems, Astrophys. Space Sci. 344 (2013), 45-50.

[27] A.J. Maciejewski, M. Przybylska, T. Stachowiak, and M. SzydŁowski, Global integrability of cosmological scalar fields, J. Phys. A: Math. Theor. 41 (2008), 465101 (26 pp). 
[28] A.P. Markeyev, Non-linear oscillations of a satellite at 1:1:1 resonance, J. Appl. Math. Mech. 76 (2012), 36-47.

[29] J. Marsden and A. Weinstein, Reduction of symplectic manifolds with symmetry, Rep. Math. Phys. 5 (1974), 121-130.

[30] K.R. Meyer, Symmetries and integrals in mechanics, in Dynamical Systems, M.M. Peixoto ed., Academic Press, New York (1973), 259-272.

[31] K.R. Meyer And D.C. Offin, Introduction to Hamiltonian Dynamical Systems and the N-Body Problem, Third Edition. Springer International Publishing AG (2017).

[32] K.R. Meyer, J.F. Palacián, And P. Yanguas, Geometric averaging of Hamiltonian systems: periodic solutions, stability, and KAM tori, SIAM J. Appl. Dyn. Syst. 10 (2011), 817-856.

[33] K.R. Meyer, J.F. Palacián, and P. Yanguas, Singular reduction of resonant Hamiltonians, Nonlinearity 31 (2018), 2854-2894.

[34] K.R. Meyer, J.F. Palacián, and P. Yanguas, Singular reduction of high dimensional resonant Hamiltonians, in preparation.

[35] K.R. Meyer And D.S. Schmidt, Periodic orbits near $L_{4}$ for mass ratios near the critical mass ratio of Routh, Celestial Mech. 4 (1971), 99-109.

[36] J. Mosen, Regularization of Kepler's problem and the averaging method on a manifold, Commun. Pure Appl. Math. XXIII (1970), 609-636.

[37] D.N. Page, Will entropy decrease if the Universe recollapses?, Phys. Rev. D 32 (1985), 2496-2499.

[38] J.F. Palacián, C. Vidal, J. Vidarte, And P. Yanguas, Periodic solutions and KAM tori in a triaxial potential, SIAM J. Appl. Dyn. Syst. 16 (2017) 159-187.

[39] G. REEB, Sur les solutions périodiques de certains systèmes différentiels perturbés, Canadian J. Math. 3 (1951) 339-362.

[40] G. ReEB, Sur certaines propriétés topologiques des trajectoires des systèmes dynamiques, Acad. Roy. Sci. Lett. et Beaux-Arts de Belgique. Cl. des Sci. Mém. in $8^{\circ}$, Ser. 2, 27 (1952), No. 9.

[41] H.P. Robertson, Kinematics and world structure, Astrophysical Journal 82 (1935), 284-301.

[42] H.P. Robertson, Kinematics and world structure II, Astrophysical Journal 83 (1936), 187-201.

[43] H.P. Robertson, Kinematics and world structure III, Astrophysical Journal 83 (1936), 257-271.

[44] J.A. Sanders, F. Verhulst and J. Murdock, Averaging Methods in Nonlinear Dynamical Systems, Second Edition. Springer, New York (2007).

[45] F. DOs SANTOS AND C. VIDAL, Stability of equilibrium solutions of Hamiltonian systems with $n$-degrees of freedom and single resonance in the critical case, J. Differential Equations 264 (2018), 5152-5179.

[46] J.-C. VAN DER MeER, The Hamiltonian Hopf Bifurcation. Lecture Notes in Math. 1160. Springer-Verlag, New York (1985).

[47] J. Vidarte, Averaging, Reduction and Reconstruction in Hamiltonian Systems and Applications to Problems of Celestial Mechanics. Ph.D. Thesis, Universidad del Bío-Bío, Concepción (2017).

[48] A.G. Walker, On Milne's theory of world-structure, Proc. Lond. Math. Soc. Ser. 2, 42 (1937), 90-127.

[49] V.A. Yakubovich And V.M. Starzhinskit, Linear Differential Equations with Periodic Coefficients. John Wiley \& Sons, New York (1975).

[50] P. Yanguas, J.F. Palacián, K.R. Meyer, and H.S. Dumas, Periodic solutions in Hamiltonian systems, averaging, and the Lunar problem, SIAM J. Appl. Dyn. Syst. 7 (2008), 311-340.

(J.F. Palacián) Departamento de Estadística, Informática y Matemáticas and Institute for Advanced Materials, Universidad Pública de Navarra, 31006 Pamplona, Spain

E-mail address: palacian@unavarra.es

(C. Vidal) Grupo de Investigación en Sistemas Dinámicos y Aplicaciones-Gisda, Departamento de Matemática, Facultad de Ciencias, Universidad del Bío-Bío, Concepción, Viit Región, Chile

E-mail address: clvidal@ubiobio.cl

(J. Vidarte) Departamento de Matemática, Facultad de Ciencias, Universidad del Bío-Bío, ConCEPCión, VIII Región, CHILE

E-mail address: edderleomat@gmail.com

(P. Yanguas) Departamento de Estadística, Informática y Matemáticas and Institute for Advanced Materials, Universidad Pública de Navarra, 31006 Pamplona, Spain

E-mail address: yanguas@unavarra.es 